\title{
RADIAL COMPONENTS, PREHOMOGENEOUS VECTOR SPACES, AND RATIONAL CHEREDNIK ALGEBRAS
}

\author{
THIERRY LEVASSEUR
}

\begin{abstract}
Let $(\tilde{G}: V)$ be a finite dimensional representation of a connected reductive complex Lie group $(\tilde{G}: V)$. Denote by $G$ the derived subgroup of $\tilde{G}$ and assume that the categorical quotient $V / / G$ is one dimensional, i.e. $\mathbb{C}[V]^{G}=$ $\mathbb{C}[f]$ for a non constant polynomial $f$. In this situation there exists a homomorphism $\mathrm{rad}: \mathcal{D}(V)^{G} \rightarrow A_{1}(\mathbb{C})$, the radial component map, where $A_{1}(\mathbb{C})$ is the first Weyl algebra. We show that the image of rad is isomorphic to the spherical subalgebra of a rational Cherednik algebra whose multiplicity function is defined by the roots of the Bernstein-Sato polynomial of $f$. In the case where $(\tilde{G}: V)$ is also multiplicity free we describe the kernel of $\operatorname{rad}$ and prove a Howe duality result between representations of $G$ occuring in $\mathbb{C}[V]$ and lowest weight modules over the Lie algebra generated by $f$ and the "dual" differential operator $\Delta \in S(V)$; this extends results of $\mathrm{H}$. Rubenthaler obtained when $(\tilde{G}: V)$ is a parabolic prehomogeneous vector space. If $(\tilde{G}: V)$ satisfies a Capelli type condition, some applications are given to holonomic and equivariant $D$-modules on $V$. These applications are related to results proved by M. Muro or P. Nang in special cases of the representation $(\tilde{G}: V)$.
\end{abstract}

\section{Contents}

1. Introduction

2. Rational Cherednik Algebras of Rank One

2.1. The spherical subalgebra and its restriction

2.2. The one dimensional case

2.3. Algebras similar to $U(\mathfrak{s l}(2))$

3. Representations with a one dimensional quotient 9

3.1. Prehomogeneous vector spaces 10

3.2. PHV of rank one 11

4. Multiplicity free representations 15

4.1. Generalities 15

4.2. MF representations with a one dimensional quotient 19

4.3. A Howe duality 21

5. D-modules on some PHV 23

5.1. Representations of Capelli type 23

5.2. Application to $D$-modules 24

5.3. Solutions of invariant differential equations 27

5.4. Regular holonomic modules 28

Appendix A. Irreducible MF representations 32

References $\quad 33$

2000 Mathematics Subject Classification. 14L30, 16S32, 17B45, 20G20, 22 E46.

Key words and phrases. prehomogeneous vector space, ring of differential operators, Dunkl operator, Cherednik algebra, Capelli operator, radial component, holonomic module. 


\section{INTRODUCTION}

The base field is the field $\mathbb{C}$ of complex numbers. Let $(G: V)$ be a finite dimensional representation of a connected reductive Lie group $G$. The action of $G$ extends to various algebras: $\mathbb{C}[V]=S\left(V^{*}\right)$ the polynomial functions on $V, \mathcal{D}(V)$ the differential operators on $V$ with coefficients in $\mathbb{C}[V]$ and $S(V)$ identified with differential operators on $V$ with constant coefficients. Recall that $\mathcal{D}(V) \cong \mathbb{C}[V] \otimes S(V)$ as a $(\mathbb{C}[V], G)$-module and that $g \in G$ acts on $D \in \mathcal{D}(V)$ by $(g . D)(\varphi)=g \cdot D\left(g^{-1} \cdot \varphi\right)$ for all $\varphi \in \mathbb{C}[V]$. We thus obtain algebras of invariants $\mathbb{C}[V]^{G}, S(V)^{G}$ and $\mathcal{D}(V)^{G}$. Then $\mathbb{C}[V]^{G}$ is (by definition) the algebra of regular functions on the categorical quotient $V / / G$ and one can define the algebra $\mathcal{D}(V / / G)$ of differential operators on this quotient (see [12] or [32]).

If $D \in \mathcal{D}(V)^{G}$ and $f \in \mathbb{C}[V]^{G}$ one obviously has $D(f) \in \mathbb{C}[V]^{G}$; this gives an algebra homomorphism:

$$
\mathcal{D}(V)^{G} \longrightarrow \mathcal{D}(V / / G), \quad D \mapsto\left\{f \mapsto D(f), f \in \mathbb{C}[V]^{G}\right\} .
$$

In general $V / / G$ is singular and $\mathcal{D}(V / / G)$ is difficult to describe. We will be interested here in the case where $V / / G$ is smooth, i.e. isomorphic to $\mathbb{C}^{\ell}$ for some $\ell \in \mathbb{N}$, in which case $\mathcal{D}(V / / G)$ is isomorphic to the Weyl algebra $A_{\ell}(\mathbb{C})$. More precisely, we want to work with polar representations as defined by J. Dadoc and V. Kac in [5]. In this case there exists a Cartan subspace $\mathfrak{h} \subset V$, a finite subgroup $W \subset \operatorname{GL}(\mathfrak{h})$ generated by complex reflections $\left(W \simeq N_{G}(\mathfrak{h}) / Z_{G}(\mathfrak{h})\right)$, such that the restriction map $\psi: \mathbb{C}[V]^{G} \rightarrow \mathbb{C}[\mathfrak{h}]^{W}, \psi(f)=f_{\mid \mathfrak{h}}$, is an isomorphism. Thus $\psi$ yields the isomorphism $V / / G \stackrel{\sim}{\sim} \mathfrak{h} / W \equiv \mathbb{C}^{\ell}$ and, consequently, an isomorphism $\mathcal{D}(V / / G) \stackrel{\sim}{\longrightarrow} \mathcal{D}(\mathfrak{h} / W) \equiv A_{\ell}(\mathbb{C})$. Recall that among the polar representations one finds two important classes:

- the representations with a one dimensional quotient, i.e. $\operatorname{dim} V / / G=1$;

- the class of "theta groups".

In the latter case there exists a semisimple Lie algebra $\mathfrak{s}$ and a $\mathbb{Z}_{m}$-grading $\mathfrak{s}=\bigoplus_{i=0}^{m-1} \mathfrak{s}_{i}$ such that $(G: V)$ identifies with the representation of the adjoint group of $\mathfrak{s}_{0}$ acting on $\mathfrak{s}_{1}$. This generalizes the case of symmetric pairs $(G: V)=(K: \mathfrak{p})$ where (with obvious notation) $\mathfrak{s}=\mathfrak{k} \oplus \mathfrak{p}$ is the decomposition associated to a complexified Cartan involution on $\mathfrak{s}$. Here $\mathfrak{h} \subset \mathfrak{p}$ is a usual Cartan subspace and $W$ is a Weyl group (cf. [15]).

Return to a general polar representation $(G: V)$. Combining the morphism $\mathcal{D}(V)^{G} \rightarrow \mathcal{D}(V / / G)$ with the isomorphism $\mathcal{D}(V / / G) \stackrel{\sim}{\longrightarrow} \mathcal{D}(\mathfrak{h} / W)$ we get the radial component map:

$$
\operatorname{rad}: \mathcal{D}(V)^{G} \longrightarrow \mathcal{D}(\mathfrak{h} / W), \quad \operatorname{rad}(D)(f)=\psi\left(D\left(\psi^{-1}(f)\right)\right), \quad f \in \mathbb{C}[\mathfrak{h}]^{W} .
$$

The morphism rad has proved to be useful in the representation theory of semisimple Lie algebras, or symmetric pairs $(\mathfrak{s}: \mathfrak{k})$ as above, see, e.g. [15, 54, 29, 30]. Two obvious questions arise: describe the algebra $R=\operatorname{Im}(\mathrm{rad}) \subset \mathcal{D}(\mathfrak{h} / W)$ and the ideal $J=\operatorname{Ker}(\mathrm{rad}) \subset \mathcal{D}(V)^{G}$. Some answers have been given in particular cases, see for example [30, 50], and it is expected that the algebra $\mathcal{D}(V)^{G} / J$ has a representation theory similar to that of factors of enveloping algebras of semisimple Lie algebras (cf. [51]).

It is known that in the case $(G: V)=(K: \mathfrak{p})$ of a symmetric pair, the subalgebra $\operatorname{rad}\left(S(\mathfrak{p})^{K}\right)$ of $R$ can be described via the introduction Dunkl operators $[7,14,6,53]$. It is therefore natural to use rational Cherednik algebras $[8,9,11]$ to describe $R$. Recall that to each complex reflection group $(W: \mathfrak{h})$ is associated an algebra $\mathcal{H}(k)$ where $k$ is a "multiplicity function" on the set of reflecting hyperplanes in $\mathfrak{h}$. Denoting by $\mathfrak{h}^{\text {reg }}$ the complement of these hyperplanes, $\mathcal{H}(k)$ is a subalgebra of the crossed product $\mathcal{D}\left(\mathfrak{h}^{\text {reg }}\right) \rtimes \mathbb{C} W$ generated by $\mathbb{C}[\mathfrak{h}], \mathbb{C} W$ and a 
subalgebra $\mathbb{C}\left[T_{1} \ldots, T_{\ell}\right] \cong S(\mathfrak{h})$ where each $T_{i}$ is a (generalized) Dunkl operator, see $\S 2.1$ for details. If $\mathrm{e}=\frac{1}{W} \sum_{w \in W} w \in \mathbb{C} W$ is the trivial idempotent, $\mathrm{e} \mathcal{H}(k) \mathrm{e}$ is called the spherical subalgebra. Then one can show that there exists an injective homomorphism

$$
\text { res : } \mathrm{e} \mathcal{H}(k) \mathrm{e} \longrightarrow \mathcal{D}(\mathfrak{h} / W)
$$

and we obtain in this way a family $U(k)=\operatorname{res}(\mathrm{e} \mathcal{H}(k) \mathrm{e})$ of subalgebras of $\mathcal{D}(\mathfrak{h} / W)$. One would like to obtain information on $R$ by answering the following question:

Does there exist a multiplicity function $k$ such that $R=U(k)$ ?

For instance, suppose that $(G: V)=(K: \mathfrak{p})$ as above. The reflecting hyperplanes are then parametrised by elements of the reduced root system $\mathrm{R}$ defined by $(\mathfrak{s}, \mathfrak{h})$ and one defines a multiplicity function by:

$$
k(\alpha)=\frac{1}{2}\left(\operatorname{dim} \mathfrak{s}^{\alpha}+\operatorname{dim} \mathfrak{s}^{2 \alpha}\right), \quad \alpha \in \mathrm{R},
$$

where $\mathfrak{s}^{\beta}$ is the root space associated to the $\operatorname{root} \beta$. For this choice of $k$ one can prove [31]:

Theorem (L-Stafford). One has $R=\operatorname{Im}(\operatorname{rad})=U(k) \cong \mathrm{e} \mathcal{H}(k) \mathrm{e}$.

Our aim in this work is to analyse a simpler case, $G$ semisimple and $\operatorname{dim} V / / G=$ $\operatorname{dim} \mathfrak{h}=1$ (hence $W \simeq \mathbb{Z} / n \mathbb{Z}$ ) and to give some applications of the radial component map in this situation. The function $k$ is then given by $n-1$ complex parameters $k_{1}, \ldots, k_{n-1}$, and $R, U(k)$ are subalgebras of the fist Weyl algebra $\mathbb{C}\left[z, \partial_{z}\right]$. The paper is organized as follows.

In $\$ 2$ we recall general facts about Cherednik algebras and their spherical subalgebras in the one dimensional case. We show (Proposition 2.8) that $U(k)=\widetilde{U} /(\Omega)$ where $\widetilde{U}$ is an algebra similar to $U(\mathfrak{s l}(2)$ ) (as defined in [52]) and $\Omega$ is a generator of the centre of $\widetilde{U}$. This says in particular that the representation of $U(k)$ is well understood (and already known).

In the third section we assume that $V$ is a representation of the reductive group $\tilde{G}, G$ is the derived group of $\tilde{G}$ and $\mathbb{C}[V]^{G}=\mathbb{C}[f]$ for a non constant $f$. Then it is known that: $\tilde{G}$ acts on $V$ with an open orbit, i.e. $(\tilde{G}: V)$ is a prehomogeneous vector space $(\mathrm{PHV}), S(V)^{G}=\mathbb{C}[\Delta], \Delta\left(f^{s+1}\right)=b(s) f^{s}$ where $b(s)=c(s+1)(s+$ $\left.\alpha_{1}\right) \cdots\left(s+\alpha_{n-1}\right)$ is the (Bernstein-)Sato polynomial of $f$. Choosing $k_{i}=\alpha_{i}-1+\frac{i}{n}$, $1 \leq i \leq n-1$, we prove that $R=U(k)$ (Theorem 3.9).

In section 4 we assume furthermore that the representation $(\tilde{G}: V)$ is multiplicity free (MF). By [18] this is equivalent to the fact that $\mathcal{D}(V)^{\tilde{G}}=\mathbb{C}\left[E_{0}, \ldots, E_{r}\right]$ is a commutative polynomial ring. If $\Theta$ is the Euler vector field on $V$ one can find polynomials $b_{E_{i}}(s)$ such that, if $\Omega_{i}=E_{i}-b_{E_{i}}(\Theta), J=\sum_{i=0}^{r} \mathcal{D}(V)^{G} \Omega_{i}$ (Theorem 4.11). We then give a duality (of Howe type) between representations of $G$ and lowest weight modules over the Lie algebra generated by $f$ and $\Delta$ (which is infinite dimensional when $\operatorname{deg} f \geq 3$ ). This duality recovers, and extends, results obtained by H. Rubenthaler [49] when $(\tilde{G}: V)$ is of commutative parabolic type.

In the last section we specialize further to the case where $(\tilde{G}: V)$ is of "Capelli type", i.e. $(\tilde{G}: V)$ is an irreducible MF representation such that $\mathcal{D}(V)^{\tilde{G}}$ is equal to the image of the centre of $U(\tilde{\mathfrak{g}})$ under the differential $\tau: \tilde{\mathfrak{g}} \rightarrow \mathcal{D}(V)$ of the $\tilde{G}$-action. These representations have been studied in [18], they fall into eight cases (see Appendix A). It is not difficult to see that $J=[\mathcal{D}(V) \tau(\mathfrak{g})]^{G}$ when $(\tilde{G}: V)$ is of Capelli type (Proposition 5.3) . We first apply this result to study $\mathcal{D}_{V}$-modules of the form $\mathcal{M}(g, k)=\mathcal{D}(V) /\left(\mathcal{D}(V) \tau(\mathfrak{g})+\mathcal{D}(V) q(\Theta) Q_{k}\right)$ where $q(s)$ is a polynomial and $Q_{k}=f^{k}$ or $\Delta^{k}$. We show in Theorem 5.9 that $\mathcal{M}(g, k)$ is holonomic if and only if $q(s) \neq 0$. This has the well known consequence that the 
space of hyperfunction solutions of $\mathcal{M}(g, k)$ is finite dimensional. These properties generalize results obtained by M. Muro [36, 37]. For the second application, recall first the classical fact [21] that if $(\tilde{G}: V)$ is MF, there is a finite number of $\tilde{G}$ orbits $\mathcal{O}_{i}, 1 \leq i \leq t$, in $V$. Let $\tilde{\mathcal{C}}=\bigcup_{i=1}^{t} \overline{T_{O_{i}}^{*} V}$ be the union of the conormal bundles to the orbits. P. Nang has shown that, when $(\tilde{G}: V)=\left(\operatorname{SO}(n) \times \mathbb{C}^{*}: \mathbb{C}^{n}\right)$, $\left(\mathrm{GL}(n) \times \mathrm{SL}(n): \mathrm{M}_{n}(\mathbb{C})\right)$ or $\left(\mathrm{GL}(2 n): \bigwedge^{2} \mathbb{C}^{2 n}\right)$, the category $\bmod _{\tilde{\mathcal{C}}}^{\mathrm{rh}}\left(\mathcal{D}_{V}\right)$ of regular holonomic $\mathcal{D}_{V}$-modules whose characteristic variety is contained in $\tilde{\mathcal{C}}$ is equivalent to the category $\bmod ^{\theta}(R)$ of finitely generated $R$-modules on which $\theta=z \partial_{z}$ acts locally finitely. These representations are of Capelli type. We conjecture (see Conjecture 5.17) that when $(\tilde{G}: V)$ is of Capelli type the category $\bmod ^{G \times \mathbb{C}^{*}}\left(\mathcal{D}_{V}\right)$ of $\left(G \times \mathbb{C}^{*}\right)$-equivariant $\mathcal{D}_{V}$-modules is equivalent to $\bmod ^{\theta}(R)$. If $G$ is simply connected, $\bmod ^{G \times \mathbb{C}^{*}}\left(\mathcal{D}_{V}\right)=\bmod _{\tilde{\mathcal{C}}}^{\text {rh }}\left(\mathcal{D}_{V}\right)$ and the conjecture covers Nang's results; since $\bmod ^{\theta}(R)$ can be easily described as a quiver category (i.e. finite diagrams of linear maps) its validity would give a simple classification of $\left(G \times \mathbb{C}^{*}\right)$-equivariant $\mathcal{D}_{V}$-modules. One can observe (Proposition 5.16) that, as in [39, 41, 43], the proof of the conjecture reduces to show that any $M \in \bmod ^{G \times \mathbb{C}^{*}}\left(\mathcal{D}_{V}\right)$ is generated by its $G$-fixed points.

\section{Rational Cherednik Algebras of Rank One}

2.1. The spherical subalgebra and its restriction. In this section we summarize some of the results we will need about rational Cherednik algebras. We begin with some general facts, see for example $[9,8,11,14]$.

Let $\mathfrak{h}$ be a complex vector space of dimension $\ell$ and $W \subset \mathrm{GL}(\mathfrak{h})$ be an arbitrary complex reflection group. Denote by $\mathcal{A}=\left\{H_{s}\right\}_{s \in \mathcal{S}}$ the collection of reflecting hyperplanes associated to $W$ (where $s \in \mathcal{S} \subset W$ is a complex reflection). Let $\alpha_{s} \in \mathfrak{h}^{*}$ such that $H_{s}=\alpha_{s}^{-1}(0)$ is the reflecting hyperplane associated to $s \in \mathcal{S}$. Fix $H=H_{s} \in \mathcal{A}$; recall that the isotropy group $W_{H}=\left\{w \in W: w_{\mid H}=\operatorname{id}_{H}\right\}$ is cyclic of order $n_{H}$ (this order only depends on the conjugacy class of $s$ ). Let $\mathrm{e}_{H, i} \in \mathbb{C} W_{H}, 0 \leq i \leq n_{H}-1$, be the primitive idempotents of $\mathbb{C} W_{H}$. Fix a family

$$
k_{H_{s}, i} \in \mathbb{C}, \quad H_{s} \in \mathcal{A}, \quad 0 \leq i \leq n_{H_{s}}-1, \quad k_{H_{s}, 0}=0,
$$

of complex numbers such that $k_{H_{s}, i}=k_{H_{t}, i}$ if $s, t \in \mathcal{S}$ are conjugate. Such a family $k=\left(k_{H, i}\right)_{H, i}$ is called a multiplicity function. Let $\mathfrak{h}^{\text {reg }}$ be the complement of $\bigcup_{s \in \mathcal{S}} H_{s}$ and set $\pi=\prod_{s \in \mathcal{S}} \alpha_{s}$. The group $W$ acts naturally on $\mathbb{C}[\mathfrak{h}]=S\left(\mathfrak{h}^{*}\right)$, $\mathbb{C}\left[\mathfrak{h}^{\text {reg }}\right]=\mathbb{C}[\mathfrak{h}]\left[\pi^{-1}\right]$, hence on End $\mathbb{C} \mathbb{C}[\mathfrak{h}]$ and End $\mathbb{C} \mathbb{C}\left[\mathfrak{h}^{\text {reg }}\right]$. These actions restrict to $\mathcal{D}(\mathfrak{h})$ and $\mathcal{D}\left(\mathfrak{h}^{\text {reg }}\right)=\mathcal{D}(\mathfrak{h})\left[\pi^{-1}\right]$. Denote by $\mathcal{D}\left(\mathfrak{h}^{\text {reg }}\right) \rtimes \mathbb{C} W$ the crossed product of the algebra $\mathcal{D}\left(\mathfrak{h}^{\text {reg }}\right)$ by the group $W$. Recall that in that algebra we have: $w f w^{-1}=w . f, w \partial(y) w^{-1}=\partial(w . y)$ if $f \in \mathbb{C}[\mathfrak{h}]$ and $\partial(y)$ is the vector field defined by $y \in V$.

Then [8] one can introduce a subalgebra

$$
\mathcal{H}=\mathcal{H}(W, k) \subset \mathcal{D}\left(\mathfrak{h}^{\text {reg }}\right) \rtimes \mathbb{C} W
$$

generated by three parts: $\mathbb{C}[\mathfrak{h}], W, \mathbb{C}[T(y): y \in \mathfrak{h}] \cong S(\mathfrak{h})$, where $T(y)$ is a Dunkl operator defined as follows. Set $a_{H_{s}}(k)=n_{H_{s}} \sum_{i=1}^{n_{H_{s}}-1} k_{H_{s}, i} \mathrm{e}_{H_{s}, i} \in \mathbb{C} W_{H_{s}}$ and

$$
T(y)=\partial(y)+\sum_{H_{s} \in \mathcal{A}} \frac{\left\langle\alpha_{s}, y\right\rangle}{\alpha_{s}} a_{H_{s}}(k) \in \mathcal{D}\left(\mathfrak{h}^{\mathrm{reg}}\right) \rtimes \mathbb{C} W .
$$

Denote by res : $\mathcal{D}\left(\mathfrak{h}^{\text {reg }}\right) \rtimes \mathbb{C} W \rightarrow$ End $_{\mathbb{C}} \mathbb{C}\left[\mathfrak{h}^{\text {reg }}\right]$ the representation given by the natural action of $W$ and $\mathcal{D}\left(\mathfrak{h}^{\text {reg }}\right)$ on $\mathbb{C}\left[\mathfrak{h}^{\text {reg }}\right]$. As observed in $[8, \S 2.5]$ (see also [9, Proposition 4.5]) $\operatorname{res}(\mathcal{H}) \subset \operatorname{End}_{\mathbb{C}} \mathbb{C}[\mathfrak{h}]$ and this gives a natural structure of faithful 
$\mathcal{H}$-module on $\mathbb{C}[\mathfrak{h}]$, i.e. we have an injective homomorphism:

$$
\text { res }: \mathcal{H} \longrightarrow \operatorname{End}_{\mathbb{C}} \mathbb{C}[\mathfrak{h}] \text {. }
$$

The group $W$ acts on $\mathcal{H}, \mathcal{D}\left(\mathfrak{h}^{\text {reg }}\right) \rtimes \mathbb{C} W$ and $\operatorname{End}_{\mathbb{C}} \mathbb{C}[\mathfrak{h}]$ by conjugation, i.e $w . u=$ $w u w^{-1}$. Denote by $\mathcal{H}^{W} \subset\left(\mathcal{D}\left(\mathfrak{h}^{\text {reg }}\right) \rtimes \mathbb{C} W\right)^{W}$ and $\left(\text { End }_{\mathbb{C}} \mathbb{C}[\mathfrak{h}]\right)^{W}$ the algebras of invariants under this action. Notice that if $u \in \mathcal{H}, w \in W$ and $f \in \mathbb{C}[\mathfrak{h}]$, we have: $\operatorname{res}(w \cdot u)(f)=\operatorname{res}\left(w u w^{-1}\right)(f)=\operatorname{res}(w u)\left(w^{-1} \cdot f\right)=w \cdot \operatorname{res}(u)\left(w^{-1} \cdot f\right)=$ $(w \cdot \operatorname{res}(u))(f)$. Thus the homomorphism res is $W$-equivariant and, in particular, res : $\mathcal{H}^{W} \rightarrow\left(\operatorname{End}_{\mathbb{C}} \mathbb{C}[\mathfrak{h}]\right)^{W}$. Therefore $w \cdot \operatorname{res}(u)(f)=w \cdot \operatorname{res}(u)\left(w^{-1} \cdot f\right)=$ $(w \cdot \operatorname{res}(u))(f)=\operatorname{res}(u)(f)$, for all $u \in \mathcal{H}^{W}, w \in W, f \in \mathbb{C}[\mathfrak{h}]^{W}$. We have obtained the following representation of $\mathcal{H}^{W}$ on $\mathbb{C}[\mathfrak{h}]^{W}$ :

$$
\text { res : } \mathcal{H}^{W} \longrightarrow \operatorname{End}_{\mathbb{C}} \mathbb{C}[\mathfrak{h}]^{W} \text {. }
$$

(This morphism is not injective when $W \neq\{1\}$.) Let

$$
\mathrm{e}=\frac{1}{|W|} \sum_{w \in W} w
$$

be the trivial idempotent and define the spherical subalgebra:

$$
\mathrm{e} \mathcal{H} \mathrm{e}=\mathrm{e}^{W} \subset \mathcal{H}^{W} .
$$

Observe that e $\mathcal{H}$ e is an algebra whose unit is equal to e. From the previous

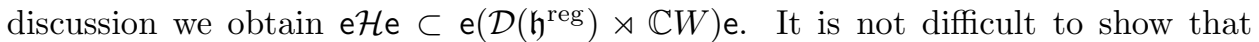
$\mathrm{e}\left(\mathcal{D}\left(\mathfrak{h}^{\text {reg }}\right) \rtimes \mathbb{C} W\right) \mathrm{e}=\mathrm{e} \mathcal{D}\left(\mathfrak{h}^{\text {reg }}\right)^{W} \cong \mathcal{D}\left(\mathfrak{h}^{\text {reg }}\right)^{W}$. It follows that $u \in \mathrm{e}^{W}$ can be written $u=$ ed for some $d \in \mathcal{D}\left(\mathfrak{h}^{\text {reg }}\right)^{W}$, hence $\operatorname{res}(u)(f)=d(f)$ for all $f \in \mathbb{C}[\mathfrak{h}]^{W}$. This implies that $\operatorname{res}(u) \in \operatorname{End}_{\mathbb{C}} \mathbb{C}[\mathfrak{h}]^{W}$ acts as the differential operator $d$ on $\mathbb{C}[\mathfrak{h}]^{W}$. Consequently, $\left.\operatorname{res}(u) \in \mathcal{D}(\mathfrak{h} / W)=\mathcal{D}\left(\mathbb{C}[\mathfrak{h}]^{W}\right) \subset \mathcal{D}\left(\mathfrak{h}^{\text {reg }} / W\right) \cong \mathcal{D}(\mathfrak{h})^{\text {reg }}\right)^{W}$. Furthermore it is easy to see that $d=0$ on $\mathbb{C}[\mathfrak{h}]^{W}$ implies $d=0$, hence $u=0$. In conclusion: one has the injective restriction morphism (see [14] in the case of a Weyl group):

$$
\text { res : e } \mathcal{H}^{W} \longrightarrow \mathcal{D}(\mathfrak{h} / W), \quad \forall f \in \mathbb{C}[\mathfrak{h}]^{W}, D \in \mathcal{H}^{W}, \operatorname{res}(\mathrm{e} D)(f)=\operatorname{res}(D)(f) \text {. }
$$

We set:

$$
U=U(W, k)=\operatorname{res}\left(\mathrm{e}^{W}\right) \subset \mathcal{D}(\mathfrak{h} / W)
$$

2.2. The one dimensional case. We now go the most simplest case of the previous construction: the case when $\ell=\operatorname{dim} \mathfrak{h}=1$.

Notation. Let $\mathfrak{h}=\mathbb{C} v$ be a one dimensional vector space and $W \subset \mathrm{GL}(\mathfrak{h})$ be a finite subgroup of order $n$. We adopt the following notation.

- $\mathbb{C}[\mathfrak{h}]=S\left(\mathfrak{h}^{*}\right)=\mathbb{C}[x],\langle x, v\rangle=1, \mathcal{D}(\mathfrak{h})=\mathbb{C}\left[x, \partial_{x}\right] ;$

- $W=\langle w\rangle \simeq \mathbb{Z} / n \mathbb{Z}, w \cdot x=\zeta x$ where $\zeta$ is a primitive $n$-th root of unity;

- $\mathbb{C}[\mathfrak{h}]^{W}=\mathbb{C}[z], z=x^{n}, \mathcal{D}(\mathfrak{h} / W)=\mathbb{C}\left[z, \partial_{z}\right], \theta=z \partial_{z} ;$

- $\mathrm{e}_{0}=\mathrm{e}, \mathrm{e}_{1}, \ldots, \mathrm{e}_{n-1} \in \mathbb{C} W$ are the primitive idempotents (hence $\mathrm{e}_{i}=$ $\left.\frac{1}{n} \sum_{j=0}^{n-1} \zeta^{i j} w^{j}\right)$

- $k_{0}=0, k_{1}, \ldots, k_{n-1} \in \mathbb{C}$;

- $T=T(v)=\partial_{x}+\frac{n}{x} \sum_{i=1}^{n-1} k_{i} \mathrm{e}_{i} \in \mathbb{C}\left[x^{ \pm 1}, \partial_{x}\right] \rtimes \mathbb{C} W$;

- if $p(s) \in \mathbb{C}[s]$ is a polynomial, set: $\tau p(s)=p(s+1)-p(s), \tau^{j+1} p(s)=$ $\tau\left(\tau^{j} p\right)(s), p^{*}(s)=p(s-1)$.

The following well known lemma will prove useful (see [26] for a more general statement). 
Lemma 2.1. Let $Q \in \mathbb{C}\left[z, \partial_{z}\right]$ satisfying:

$$
\exists p \in \mathbb{Z}, \quad \forall m \in \mathbb{N}, \quad Q\left(z^{m}\right) \in \mathbb{C} z^{m+p} .
$$

Then there exists a polynomial $\varphi(s) \in \mathbb{C}[s]$ of degree $d$ such that: $Q$ has order $d$ and can be written

$$
Q=z^{p} \varphi(\theta)=\sum_{j=0}^{d} q_{j}(z) \partial_{z}^{j}
$$

where

$$
q_{j}(z)=\frac{1}{j !}\left(\tau^{j} \varphi\right)(0) z^{j+p} \quad \text { and }\left(\tau^{j} \varphi\right)(0)=0 \text { if } p+j<0 .
$$

Remark 2.2. One can define the algebra $\mathbb{C}\left[z^{\alpha}: \alpha \in \mathbb{Q}\right]$ by adjoining roots of polynomials of the form $t^{p}-z, p \in \mathbb{N}$ prime. The derivation $\partial_{z}$ is naturally defined on this algebra by $\partial_{z}\left(z^{\alpha}\right)=\alpha z^{\alpha-1}$. Let $Q$ be as in Lemma 2.1; then $Q$ extends to $\mathbb{C}\left[z^{\alpha}: \alpha \in \mathbb{Q}\right]$ by $Q\left(z^{\alpha}\right)=\sum_{j} q_{j}(z) \partial_{z}\left(z^{\alpha}\right)=\varphi(\alpha) z^{\alpha+p}$.

The next lemma is straightforward by direct computation.

Lemma 2.3. The following formulas hold:

(a) $\left[\mathrm{e}_{i}, x\right]=x\left(\mathrm{e}_{i+1}-\mathrm{e}_{i}\right)$ (where $\left.\mathrm{e}_{n}=\mathrm{e}_{0}=\mathrm{e}\right)$;

(b) $[T, x]=1+n \sum_{i=1}^{n-1} k_{i}\left(\mathrm{e}_{i+1}-\mathrm{e}_{i}\right)=1+n \sum_{i=0}^{n-1}\left(k_{i}-k_{i+1}\right) \mathrm{e}_{i}$;

(c) $w T w^{-1}=\zeta^{-1} T$;

(d) let $p \in \mathbb{N}$ and define $q \in\{0, \ldots, n-1\}$ by $p+q \equiv 0(\bmod n)$, then $T\left(x^{p}\right)=$ $\left(n k_{q}+p\right) x^{p-1}$

(e) let $1 \leq j \leq n$ and $s \in \mathbb{N}$, then

$$
T^{j}\left(x^{s n}\right)=\prod_{i=1}^{j}\left(n k_{i-1}+s n-i+1\right) x^{s n-j},
$$

in particular $(T / n)^{n}\left(z^{s}\right)=\prod_{i=0}^{n-1}\left(s+k_{i}-i / n\right) z^{s-1}$.

We now introduce the rational Cherednik algebra, and its spherical subalgebra, in the rank one case.

Definition 2.4. The rational Cherednik algebra associated to $W$ with parameters $k_{i}, 0 \leq i \leq n-1$, is the subalgebra of $\mathbb{C}\left[x^{ \pm 1}, \partial_{x}\right] \rtimes \mathbb{C} W$ defined by:

$$
\mathcal{H}=\mathcal{H}\left(W, k_{0}, \ldots, k_{n-1}\right)=\mathbb{C}\langle x, T, w\rangle
$$

Its spherical subalgebra is e $\mathcal{H}$.

Observe that when $n=1$ (i.e. $W$ trivial) the algebra $\mathcal{H}=$ e $\mathcal{H}$ e is nothing but $\mathcal{D}(\mathfrak{h})=\mathbb{C}\left[x, \partial_{x}\right]$ and all the results we are going to obtain are in this case obvious. We therefore will only be interested in the case $n \geq 2$.

It is easily seen that:

- $\mathrm{e} \mathcal{H} \mathrm{e}=\mathrm{e} \mathcal{H}^{W}=\mathbb{C}\left\langle\mathrm{e}, \mathrm{e} x^{n}, \mathrm{e}(T / n)^{n}, \mathrm{e} x T / n\right\rangle$;

- the image $U=\operatorname{res}(\mathrm{e} \mathcal{H e})$ of the injective homomorphism, defined in (2.2),

$$
\text { res : e } \mathcal{H}^{W} \longrightarrow \mathcal{D}(\mathfrak{h} / W)=\mathbb{C}\left[z, \partial_{z}\right]
$$

is generated by $z, \operatorname{res}\left(\mathrm{e}(T / n)^{n}\right)$ and $\operatorname{res}(\mathrm{e} x T / n)$.

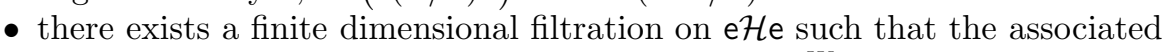
graded algebra $\operatorname{gr}(\mathrm{e} \mathcal{H} \mathrm{e})$ is isomorphic to $S\left(\mathfrak{h}^{*} \times \mathfrak{h}\right)^{W} \equiv \mathbb{C}[X, Y, S] /(X Y-$ $\left.S^{n}\right)$, cf. [9, p. 262] (one has $X \equiv \operatorname{gr}\left(\mathrm{e} x^{n}\right), Y \equiv \operatorname{gr}\left(\mathrm{e} T^{n}\right), S \equiv \operatorname{gr}(\mathrm{e} x T)$ ). 
Fix a constant $c \in \mathbb{C}^{*}$ and set:

$$
\begin{gathered}
\lambda_{i}=k_{i}-\frac{i}{n}, \quad b^{*}(s)=c \prod_{i=0}^{n-1}\left(s+\lambda_{i}\right), \quad b(s)=b^{*}(s+1)=c \prod_{i=0}^{n-1}\left(s+\lambda_{i}+1\right) \\
v(s)=-2 b(-s), \quad \psi(s)=\frac{1}{2}(\tau v)(s)=b(-s)-b(-s-1) .
\end{gathered}
$$

Proposition 2.5. Set $\delta=c \operatorname{res}\left(\mathrm{e}(T / n)^{n}\right)$. Then $U=\operatorname{res}(\mathrm{e} \mathcal{H e})=\mathbb{C}[z, \theta, \delta]$ and one has:

(1) $\delta=z^{-1} b^{*}(\theta)=\sum_{j=1}^{n} \frac{1}{j !}\left(\tau^{j} b^{*}\right)(0) z^{j-1} \partial_{z}^{j}$;

(2) $\operatorname{res}(\mathrm{e} x T / n)=\theta$;

(3) $[\delta, z]=\psi(-\theta)=b(\theta)-b(\theta-1)=\left(\tau b^{*}\right)(\theta)$;

(4) $[\theta, z]=z,[\theta, \delta]=-\delta$;

(5) $2 z \delta+v(-\theta+1)=2\left(z \delta-b^{*}(\theta)\right)=0$.

Proof. The equality $U=\mathbb{C}[z, \theta, \delta]$ is clear.

(1) From the definition of the map res, cf. (2.2), and Lemma 2.3(e) we deduce that $\delta\left(z^{s}\right)=c(T / n)^{n}\left(z^{s}\right)=b^{*}(s) z^{s-1}$. The claim therefore follows from Lemma 2.1 applied to $Q=\delta, \varphi=b^{*}$ and $p=-1$.

(2) By Lemma 2.3(e) again we get that $\operatorname{res}(x T)\left(z^{s}\right)=x T\left(x^{s n}\right)=s n x^{s n}=n s z^{s}$, i.e. $\operatorname{res}(\mathrm{e} x T / n)=\theta$.

(3) Using (1) we obtain that $[\delta, z]\left(z^{s}\right)=\left(\tau b^{*}\right)(s) z^{s}=\left(\tau b^{*}\right)(\theta)\left(z^{s}\right)$, hence $[\delta, z]=$ $\left(\tau b^{*}\right)(\theta)$.

The formulas in (4) and (5) are obvious.

2.3. Algebras similar to $U(\mathfrak{s l}(2))$. We recall here the definition, and some properties, of the algebras similar to $U(\mathfrak{s l}(2))$ introduced in [20] and [52].

Let $\psi(s) \in \mathbb{C}[s]$ be an arbitrary polynomial of degree $\geq 1$ and write $\psi=\frac{1}{2} \tau v$ for some $v \in \mathbb{C}[s]$ of degree $n \geq 2$. Define a $\mathbb{C}$-algebra $\widetilde{U}$ by generators and relations as follows (cf. [52]):

$\widetilde{U}=\widetilde{U}(\psi)=C\langle A, B, H\rangle, \quad[A, B]-\psi(H)=0, \quad[H, A]-A=0, \quad[H, B]+B=0$.

Note that when $\operatorname{deg} \psi=1$, i.e. $n=2$, one has $\widetilde{U}=U(\mathfrak{s l}(2))$. The algebra $\widetilde{U}$ has the following properties, see $[52,19,38]$.

- The centre of $\widetilde{U}$ is $Z(\widetilde{U})=\mathbb{C}[\Omega], \Omega=2 B A+v(H+1)=2 A B+v(H)$.

- For $\lambda \in \mathbb{C}$ one defines the "Verma module" $M(\lambda)=\widetilde{U} \otimes_{\mathbb{C}[H, A]} \mathbb{C}_{\lambda}$, where $\mathbb{C}_{\lambda}$ is the one dimensional module associated to $\lambda$ over the solvable Lie algebra $\mathbb{C} A+\mathbb{C} H$.

- Each $M(\lambda)$ has a unique simple quotient $L(\lambda)$ and any finite dimensional $\widetilde{U}$-module is of the form $L(\lambda)$ for some $\lambda$.

- The primitive ideals of $\widetilde{U}$ are the annihilators ann $L(\lambda)$; the minimal primitive ideals are the ann $M(\lambda)=(\Omega-v(\lambda+1))$, they are completely prime. If $I$ is an ideal strictly containing ann $M(\lambda)$, then $\operatorname{dim}_{\mathbb{C}} \widetilde{U} / I$ is finite.

- One can define in an obvious way a category $\mathcal{O}$ for $\widetilde{U}$ which decomposes as:

$$
\mathcal{O}=\bigsqcup_{\alpha} \mathcal{O}_{\alpha}, \quad \mathcal{O}_{\alpha}=\left\{M \in \mathcal{O}:(\Omega-\alpha)^{k} M=0 \text { for some } k\right\}
$$

Moreover $\mathcal{O}_{\alpha} \equiv \bmod A$ for a finite dimensional $\mathbb{C}$-algebra $A$.

The representation theory of the algebras $\widetilde{U} /(\Omega-v(\lambda+1))$ is therefore quite well understood.

We will be interested in the algebra $U=\widetilde{U} /(\Omega)=\mathbb{C}[a, b, h]$ where $a, b, h$ are the classes of $A, B, H$. We have in $U$ :

$$
[a, b]=\psi(h)=\frac{1}{2}(\tau v)(h), \quad[h, a]=a, \quad[h, b]=-b, \quad 2 a b=-v(h) .
$$


For simplicity we will assume that $v(1)=0$. Recall then that $M(0) \equiv \mathbb{C}[b]$ is a faithful $U$-module where $h \cdot b^{k}=-k b^{k}, b . b^{k}=b^{k+1}, a \cdot b^{k}=\left(\sum_{i=1}^{k-1} \psi(-i)\right) b^{k-1}$ for all $k \geq 0$. We want to study the Lie subalgebra $\mathcal{L}$ of $(U,[]$,$) generated by the$ elements $a, b$. Recall [52] that when $n \leq 2$ this algebra is finite dimensional. The algebra $\mathcal{L}$ acts on $\mathbb{C}[b] \equiv M(0)$ and for each $i \in \mathbb{Z}$ we set:

$$
\mathcal{L}_{i}=\left\{u \in \mathcal{L}: u . b^{k} \in \mathbb{C} b^{k+i} \text { for all } k \in \mathbb{N}\right\} .
$$

Clearly: $a \in \mathcal{L}_{-1}, h \in \mathcal{L}_{0}, b \in \mathcal{L}_{1}$.

Lemma 2.6. (1) The element $h$ is transcendental over $\mathbb{C}$.

(2) For $g(h) \in \mathbb{C}[h]$ we have $[b, a g(h)]=-\frac{1}{2} \tau\left(v g^{*}\right)(h)$.

(3) There exists a sequence $\left(g_{m}(h)\right)_{m \in \mathbb{N}} \subset \mathcal{L} \cap \mathbb{C}[h]$ such that $\operatorname{deg} g_{m}=(m+1) n-$ $(2 m+1)$. In particular, $\operatorname{deg} g_{m}<\operatorname{deg} g_{m+1}$ when $n \geq 3$.

Proof. (1) This follows, for example, from $g(h) \cdot b^{k}=g(-k) b^{k}$ in $M(0)$ for all $g(h) \in$ $\mathbb{C}[h] \subset U$.

(2) Recall [52, Appendix] that $[b, g(h)]=b(g(h)-g(h-1))$ and $[g(h), a]=a \tau(g)(h)$. Thus:

$$
\begin{aligned}
{[b, a g(h)] } & =[b, a] g(h)+a[b, g(h)]=-\frac{1}{2}(\tau v)(h) g(h)+a b(g(h)-g(h-1)) \\
& =-\frac{1}{2}(v(h+1)-v(h)) g(h)-\frac{1}{2} v(h)(g(h)-g(h-1)) \\
& =-\frac{1}{2}(v(h+1) g(h)-v(h) g(h-1)) \\
& =-\frac{1}{2}\left(\tau\left(v g^{*}\right)\right)(h),
\end{aligned}
$$

as desired.

(3) We start with $g_{0}(h)=\psi(h)=[a, b] \in \mathcal{L}$. Then, $\operatorname{deg} g_{0}=n-1$ and, by $(2)$, $\left[b, a\left(\tau g_{0}\right)(h)\right]=-\frac{1}{2} \tau\left(v\left(\tau g_{0}\right)^{*}\right)(h) \in \mathcal{L}$. We thus set $g_{1}(h)=-\frac{1}{2} \tau\left(v\left(\tau g_{0}\right)^{*}\right)(h) ;$ note that $\operatorname{deg} \tau\left(v\left(\tau g_{0}\right)^{*}\right)=\operatorname{deg} v+\operatorname{deg} \tau g_{0}-1=2 n-3$. Suppose that $g_{m}(h) \in \mathcal{L}$ has been obtained; then $\left[g_{m}(h), a\right]=a\left(\tau g_{m}\right)(h) \in \mathcal{L}$ and $\left[b,\left[g_{m}(h), a\right]\right]=-\frac{1}{2} \tau\left(v\left(\tau g_{m}\right)^{*}\right)(h) \in$ $\mathcal{L}$. Set $g_{m+1}(h)=\tau\left(v\left(\tau g_{m}\right)^{*}\right)(h)$. We have $\operatorname{deg} g_{m+1}=\operatorname{deg} v+\operatorname{deg} g_{m}-2=$ $(m+1) n-(2 m+1)+n-2=(m+2) n-(2(m+1)+1)$, hence the result.

Proposition 2.7. Assume that $n \geq 3$. Then $\operatorname{dim}_{\mathbb{C}} \mathcal{L}_{i}=\infty$ for all $i \in \mathbb{Z}$.

Proof. When $i=0$ the claim follows from Lemma 2.6(1). Suppose that $i>0$. Let $\left(g_{m}(h)\right)_{m} \subset \mathcal{L}_{0}$ be as in Lemma 2.6(3). We will now show that $a^{i}\left(\tau^{i} g_{m}\right)(h) \in \mathcal{L}_{-i}$ for all $m$. Since $\operatorname{deg} g_{m+1}>\operatorname{deg} g_{m}$ (because $n \geq 3$ ) the elements $a^{i}\left(\tau^{i} g_{m}\right)(h)$ are linearly independent in the domain $U$. We argue by induction on $i$. When $i=1$ the claim follows from $\left[a, g_{m}(h)\right]=a\left(\tau g_{m}\right)(h) \in \mathcal{L}_{-1}$ for all $m$. Assume that $a^{i}\left(\tau^{i} g_{m}\right)(h) \in \mathcal{L}_{-i}$ for all $m$, then: $\left[a, a^{i}\left(\tau^{i} g_{m}\right)(h)\right]=a^{i+1}\left(\tau^{i+1} g_{m}\right)(h) \in \mathcal{L}_{-(i+1)}$ for all $m$.

Observe that there exists an anti-automorphism of $\widetilde{U}$ given by $\varkappa(A)=B, \varkappa(B)=$ $A, \varkappa(H)=H$. It satisfies $\varkappa(\Omega)=\Omega$, therefore $\varkappa$ induces an anti-automorphism of $U$. Since $\varkappa\left(a^{i}\left(\tau^{i} g_{m}\right)(h)\right)=\left(\tau^{i} g_{m}\right)(h) b^{i} \in \mathcal{L}_{i}$, we get that $\operatorname{dim} \mathcal{L}_{i}=\infty$.

Remark. The fact that $\operatorname{dim}_{\mathbb{C}} \mathcal{L}=\infty$ when $n \geq 3$ can also be proved by using [48].

The next result shows that the spherical subalgebra e $\mathcal{H e}$ is isomorphic to a quotient $\widetilde{U} /(\Omega)$ for an an obvious choice of $\psi(s)$ :

Proposition 2.8. Let $b^{*}(s), v(s)=-2 b(-s), \psi(s)=\frac{1}{2}(\tau v)(s) \in \mathbb{C}[s]$ be as in $(2.5)$ and $(2.6)$, and denote by $U=\operatorname{res}(\mathrm{e} \mathcal{H e})=\mathbb{C}[z, \theta, \delta]$ the image of the spherical 
subalgebra under the restriction map. Let $\widetilde{U}=\mathbb{C}\langle A, B, H\rangle$ be the algebra similar to $U(\mathfrak{s l}(2))$ defined by $\psi$. Then the morphism

$$
\pi: \widetilde{U} \longrightarrow U, \quad \pi(A)=\delta, \pi(B)=z, \pi(H)=h=-\theta
$$

induces an isomorphism $\pi: \widetilde{U} /(\Omega) \stackrel{\sim}{\longrightarrow}$. In particular, there exists an isomorphism $\widetilde{U} /(\Omega) \stackrel{\sim}{\longrightarrow}$ eHe.

Proof. The existence of the surjective homomorphism $\pi$ clearly follows from (3) and (4) in Proposition 2.5. By Proposition 2.5(5), $\operatorname{Ker}(\pi)$ contains $(\Omega)$. Since $\pi(\widetilde{U})=U$ is not finite dimensional, it follows from one of the properties of $\widetilde{U}$ that $\operatorname{Ker}(\pi)=(\Omega)$.

From results of Smith [52], Musson-Van den Bergh [38], et al., one can for instance deduce the following properties of the the algebra $U \cong \mathrm{eHe}$

- The Verma modules over $U$ are the $M(\lambda)$ 's such that $v(\lambda+1)=-2 b^{*}(-\lambda)=0$, i.e. $\lambda=0, \lambda_{1}, \ldots, \lambda_{n-1}$, cf. (2.5).

$-\operatorname{dim} L(\lambda)<\infty \Longleftrightarrow b^{*}(-\lambda)=b^{*}(-\lambda+j)=0$ for some $j \in \mathbb{N}^{*}$.

- The global homological dimension of $U$ is:

$$
\begin{cases}\infty & \text { if } b^{*}(-s) \text { has multiple roots; } \\ 2 & \text { if } b^{*}(-s) \text { has no multiple root and two roots differing by some } j \in \mathbb{N}^{*} \\ 1 & \text { otherwise. }\end{cases}
$$

When a root $-\lambda$ of the polynomial $b^{*}(s)$ is a rational number one can use Remark 2.2 to realize the Verma module $M(\lambda)$ under the form $\mathbb{C}[z] z^{-\lambda}$, on which $z, \theta, \delta$ act as differential operators. This is for example the case for $\lambda=0$, where we have $M(0)=\mathbb{C}[z]$.

Examples. (1) Take $b(s)=(s+1)(s+3 / 2) \cdots(s+(n+1) / 2), \lambda_{i}=i / 2,0 \leq$ $i \leq(n-1) / 2$. Verma modules: $M\left(\lambda_{i}\right)=\mathbb{C}[z] z^{-i / 2}$ with the natural action of $z, \delta, \theta$. Irreducible finite dimensional modules: $L\left(\lambda_{i}\right)=M\left(\lambda_{i}\right) / M\left(\lambda_{i}-1\right), i \geq 2$, of dimension 1. The global dimension of $U$ is 2 .

(2) Take $b(s)=(s+1)(s+5)(s+9), \lambda_{i}=4 i, i=0,1,2$. Verma modules: $M(4 i)=$ $\mathbb{C}[z] z^{-4 i}, M(0) \subset M(4) \subset M(8)$ with quotients $L(4 i)=M(4 i) / M(4(i-1))$ simple of dimension 4 . The global dimension of $U$ is 2 .

(3) Take $b(s)=(s+1)(s+n / 2), n \geq 3, \lambda_{0}=0, \lambda_{1}=\frac{n-2}{2}$. Verma modules: $M(0)=\mathbb{C}[z], M\left(\frac{n-2}{2}\right)=\mathbb{C}[z] z^{-\frac{n-2}{2}}$. There are two cases:

- $n=2 k$, then $M(0)=\mathbb{C}[z] \subset M(k-1)=\mathbb{C}[z] z^{-(k-1)}$ with quotient $L(k-1)$ simple of dimension $k-1$; the global dimension of $U$ is 2 ;

- $n=2 k+1$, then $M(0)=\mathbb{C}[z]$ and $M\left(k-\frac{1}{2}\right)=\mathbb{C}[z] z^{-\left(k-\frac{1}{2}\right)}$ are simple; the global dimension of $U$ is 1 .

(4) Take

$$
\begin{aligned}
b(s)=(s+1)^{8} & {[(s+2 / 3)(s+4 / 3)(s+3 / 4)(s+5 / 4)(s+5 / 6)(s+7 / 6)]^{4} } \\
& \times[(s+7 / 10)(s+9 / 10)(s+11 / 10)(s+13 / 10)]^{2},
\end{aligned}
$$

$\lambda_{0}=0, \lambda_{1}=-1 / 3, \lambda_{2}=1 / 3, \lambda_{3}=-1 / 4, \lambda_{4}=1 / 4, \lambda_{5}=-1 / 6, \lambda_{6}=1 / 6, \lambda_{7}=$ $-3 / 10, \lambda_{8}=-1 / 10, \lambda_{9}=1 / 10, \lambda_{10}=3 / 10$. Verma modules: $M\left(\lambda_{i}\right)=\mathbb{C}[z] z^{-\lambda_{i}}$ which are simple $U$-modules. The global dimension of $U$ is $\infty$.

\section{REPRESENTATIONS WITH A ONE DIMENSIONAL QUOTIENT}


3.1. Prehomogeneous vector spaces. Let $\tilde{G}$ be a connected reductive complex algebraic group. We denote by $G=(\tilde{G}, \tilde{G})$ its derived subgroup, which is a connected semisimple group. Recall that $\tilde{G}=G C$ where $C=Z(\tilde{G})^{0}$, the connected component of the center of $\tilde{G}$, is a a torus.

Let $\tilde{\rho}: \tilde{G} \rightarrow \operatorname{GL}(V)$ be a finite dimensional representation of $\tilde{G}$. Recall that $f \in$ $\mathbb{C}[V]=S\left(V^{*}\right)$ is a relative invariant of $(\tilde{G}: V)$ if there exists a rational character $\chi \in \mathrm{X}(\tilde{G})$ such that $g . f=\chi(g) f$ for all $g \in \tilde{G}$.

One says, see $[25$, Chapter 2], that $(\tilde{G}: V)=(\tilde{G}, \tilde{\rho}, V)$ is a (reductive) prehomogeneous vector space (PHV) if $\tilde{G}$ has a dense orbit in $V$. We denote the complement of the dense orbit by $S$, it is called the singular set of $(\tilde{G}: V)$. Then it is known [25, Theorem 2.9] that the one-codimensional irreducible components of $V \backslash S$ are of the form $\left\{f_{i}=0\right\}, 1 \leq i \leq r$, for some relative invariants $f_{i}$. The $f_{i}$ are algebraically independent and are called the basic relative invariants of $(\tilde{G}: V)$; any relative invariant $f$ can be (up to a non-zero constant) written as $\prod_{i=1}^{r} f_{i}^{m_{i}}$. When the singular set is a hypersurface $(\tilde{G}: V)$ is called regular, cf. [25, Theorem 2.28].

Let $\tilde{\rho}^{*}: \tilde{G} \rightarrow \mathrm{GL}\left(V^{*}\right)$ be the contragredient representation. Then, see $[25$, Proposition 2.21], $\left(\tilde{G}: V^{*}\right)$ is a PHV. Recall that $S(V)=\mathbb{C}\left[V^{*}\right]$ can be identified with the algebra of constant coefficients differential operators on $V$. If $\varphi \in \mathbb{C}\left[V^{*}\right]$ we denote by $\varphi(\partial)$ the corresponding differential operator. If $f \in \mathbb{C}[V]$ is a relative invariant of degree $n$ and weight $\chi \in \mathrm{X}(\tilde{G})$, there exists a relative invariant $f^{*} \in \mathbb{C}\left[V^{*}\right]$ of degree $n$ and weight $\chi^{-1}$. The following result summarizes [25, Proposition 2.22] and [23].

Theorem 3.1 (Sato-Bernstein-Kashiwara). Under the above notation, set $\Delta=$ $f^{*}(\partial) \in S(V)$. There exists $b(s) \in \mathbb{R}[s]$ of degree $n$ such that:

(1) $b(s)=c \prod_{i=0}^{n-1}\left(s+\lambda_{i}+1\right), c>0$;

(2) $\Delta\left(f^{s+1}\right)=b(s) f^{s}$

(3) $\lambda_{i}+1 \in \mathbb{Q}_{+}^{*}, 0 \leq i \leq n-1, \lambda_{0}=0$.

The polynomial $b(s)$ is called a $b$-function of $f$. Since the form of the operator $\Delta=f^{*}(\partial)$ will be important in the proof of Theorem 3.9, we briefly indicate its expression in a particular coordinate system (see [25, p. 38]).

Denote by $\bar{a}$ the complex conjugate of $a \in \mathbb{C}$ and set $|a|^{2}=a \bar{a} \in \mathbb{R}_{+}$. Let $\tilde{K}$ be a maximal compact subgroup of $\tilde{G}$, so that $\tilde{G}=\tilde{K} \exp (i \tilde{\mathfrak{k}})$ is the complexification of $\tilde{K}$ (where $\tilde{\mathfrak{k}}=\operatorname{Lie}(\tilde{K}))$. Fix a $\tilde{K}$-invariant non-degenerate Hermitian form $\kappa$ on $V$ such that $\kappa(\lambda v, \mu w)=\bar{\lambda} \mu \kappa(v, w), \lambda, \mu \in \mathbb{C}, v, w \in V$. We choose a $\kappa$-orthonormal basis $\left\{e_{i}\right\}_{1 \leq i \leq N}$ on $V$, with dual basis $\left\{z_{i}=e_{i}^{*}\right\}_{i}$. Define

$$
\phi: V \longrightarrow V^{*}, \quad \phi(v)=v^{*}=\kappa(v,-) .
$$

In coordinates we have: $\phi\left(\sum_{i} v_{i} e_{i}\right)=\sum_{i} \bar{v}_{i} z_{i}$. Then $\phi$ is a bijective $\mathbb{C}$-antilinear map such that $\phi(h . v)=h . \phi(v)$ for all $h \in \tilde{K}$. The inverse of $\phi$, also denoted by $\phi$, is given by $\phi\left(v^{*}\right)=v$, i.e. $\phi\left(\sum_{i} a_{i} z_{i}\right)=\sum_{i} \overline{a_{i}} e_{i}$, and it also satisfies $\phi\left(h . v^{*}\right)=$ $h . \phi\left(v^{*}\right)$. We can extend $\phi$ to $S(V)=\mathbb{C}\left[V^{*}\right]$ and $S\left(V^{*}\right)=\mathbb{C}[V]$ by multilinearity. Thus we get a bijective $\tilde{K}$-equivariant $\mathbb{C}$-antilinear morphism:

$$
\phi: \mathbb{C}[V] \longrightarrow \mathbb{C}\left[V^{*}\right], \quad \phi(f)=f^{*} .
$$

Now, if $f$ is a relative invariant of $(\tilde{G}: V)$ associated to $\chi$, we obtain:

$$
h . f^{*}=h . \phi(f)=\phi(h . f)=\phi(\chi(h) f)=\overline{\chi(h)} \phi(f)=\chi(h)^{-1} f^{*}
$$

for all $h \in \tilde{K}$, which shows that $f^{*}$ is a relative invariant corresponding to $\chi^{-1}$.

The expression of $\phi(f)=f^{*}$ in the chosen basis is given as follows. If $i=$ $\left(i_{1}, \ldots, i_{N}\right) \in \mathbb{N}^{N}$ we set $|\mathbf{i}|=\sum_{j=1}^{N} i_{j}, \quad i !=\prod_{j=1}^{N} i_{j}, z^{i}=z_{1}^{i_{1}} \cdots z_{N}^{i_{N}}, e^{i}=$ 
$e_{1}^{i_{1}} \cdots e_{N}^{i_{N}}$ and $\partial^{i}=\partial_{z_{1}}^{i_{1}} \cdots \partial_{z_{N}}^{i_{N}}$. Then, if the polynomial $f$ is written

$$
f=\sum_{\left\{\mathrm{i} \in \mathbb{N}^{N}:|\mathrm{i}|=n\right\}} a_{\mathrm{i}} z^{\mathrm{i}}, \quad a_{\mathrm{i}} \in \mathbb{C},
$$

one has

$$
f^{*}=\sum_{\left\{\mathrm{i} \in \mathbb{N}^{N}:|\mathrm{i}|=n\right\}} \overline{a_{\mathrm{i}}} e^{\mathrm{i}}
$$

and therefore:

$$
\Delta=\sum_{\left\{\mathbf{i} \in \mathbb{N}^{N}:|\mathbf{i}|=n\right\}} \overline{a_{\mathrm{i}}} \partial^{\mathrm{i}}, \quad b(0)=\Delta(f)=c \prod_{i=0}^{n-1}\left(\lambda_{i}+1\right)=\sum_{\mathrm{i} \in \mathbb{N}^{N}} \mathrm{i} !\left|a_{\mathrm{i}}\right|^{2} .
$$

Remarks 3.2. 1) If $K$ is a maximal compact subgroup of the semisimple group $G$ we can embed $K$ in a maximal compact subgroup $\tilde{K}$ of $\tilde{G}$. Note that any relative invariant of $(\tilde{G}: V)$ is a $G$-invariant, and that $f$ is $G$-invariant if and only if it is $K$ invariant. The previous construction shows that there exist $\tilde{K}$-equivariant bijective $\mathbb{C}$-antilinear morphisms $\phi: S(V) \rightarrow S\left(V^{*}\right)$ and $\phi: S\left(V^{*}\right) \rightarrow S(V)$ such that $\phi \circ \phi=$ id. In particular we have $\phi\left(S\left(V^{*}\right)^{K}\right)=S(V)^{K}$, hence $\phi\left(S\left(V^{*}\right)^{G}\right)=S(V)^{G}$.

2) Observe that $\phi: V \rightarrow V^{*}$ is $K$-equivariant, but, in general, there is no $G$ module isomorphism of between $V$ and $V^{*}$. For example, in the case $(\tilde{G}: V)=$ $\left(\mathrm{GL}(n, \mathbb{C}), \bigwedge^{2} \mathbb{C}^{n}\right)$ one has $\left(\bigwedge^{2} \mathbb{C}^{n}\right)^{*} \cong \bigwedge^{n-2} \mathbb{C}^{n}$ as a $G$-module, which is not isomorphic to $\bigwedge^{2} \mathbb{C}^{n}$ when $n>2$.

3.2. PHV of rank one. Let $\tilde{G}=G C$ be as above and $(\tilde{G}: V)$ be a finite dimensional representation of $\tilde{G}$. In this subsection we make the following hypothesis:

Hypothesis A. There exists $f \in S^{n}\left(V^{*}\right)$ such that: $f \notin \mathbb{C}[V]^{\tilde{G}}$ and $\mathbb{C}[V]^{G}=\mathbb{C}[f]$.

Remarks. (1) Assume that $G$ is a semisimple group and $(G: V)$ is a finite dimensional representation of $G$ such that $\mathbb{C}[V]^{G}=\mathbb{C}[f], f \notin \mathbb{C}^{*}$. Let the group $\mathbb{C}^{*}$ act on $V$ by homotheties. Then $\left(G \times \mathbb{C}^{*}: V\right)$ satisfies the hypothesis A. Therefore one could assume without lost of generality that $C=\mathbb{C}^{*}$.

(2) Let $f$ be as in the previous hypothesis. Then, since $G$ is semisimple, the polynomial $f$ is irreducible. Let $g \in C$, then $g . f \in \mathbb{C}[V]^{G}=\mathbb{C}[f]$ and $\operatorname{deg}(g . f)=$ $\operatorname{deg}(f)$, hence $g . f=\chi(g) f$ for some $\chi(g) \in \mathbb{C}^{*}$. It follows that $\chi \in \mathrm{X}(\tilde{G})$, i.e. $f$ is a relative invariant of $(\tilde{G}: V)$; note that $\chi \neq 1$, since $f \notin \mathbb{C}[V]^{\tilde{G}}$. Assume that $f_{1}$ is another relative invariant of $(\tilde{G}: V)$. Then $f_{1} \in \mathbb{C}[V]^{G}$ is homogeneous and this implies that $f_{1}=\alpha f^{m}$ for some $\alpha \in \mathbb{C}, m \in N$.

Proposition 3.3. Let $f$ be as in hypothesis $A$.

(i) Let $\mathbb{C}(V)$ be the fraction field of $\mathbb{C}[V]$. Then one has:

$$
\mathbb{C}(V)^{G}=\mathbb{C}(f), \quad \mathbb{C}(V)^{\tilde{G}}=\mathbb{C} .
$$

In particular, $(\tilde{G}: V)$ is a $P H V$.

(ii) Let $f^{*}$ be the relative invariant obtained in $(3.3)$, then $\mathbb{C}\left[V^{*}\right]^{G}=\mathbb{C}\left[f^{*}\right]$.

(iii) The representation $(G: V)$ is polar.

Proof. (i) The equality $\mathbb{C}(V)^{G}=\mathbb{C}(f)$ follows from [46, Theorem 3.3]. By the remark (2) above, we know that $g . f=\chi(g) f$ for all $g \in \mathbb{C}$. Observe that $\mathbb{C}(V)^{\tilde{G}}=$ $\left(\mathbb{C}(V)^{G}\right)^{C}=\mathbb{C}(f)^{C}$ and let $\varphi=p(f) / q(f) \in \mathbb{C}(f)^{C}$ where $p(f), q(f) \in \mathbb{C}[f]$ are relatively prime polynomials in $f$. One easily sees that $p(f)$ and $q(f)$ are relative invariants, thus $p(f)=\alpha f^{k}$ and $q(f)=\beta f^{\ell}, \alpha, \beta \in \mathbb{C}$. It follows that $\chi^{k-\ell}=1$, hence $k=\ell$ and $\varphi \in \mathbb{C}$. From [46, Corollary, p. 156] one gets that $(\tilde{G}: V)$ is a $\mathrm{PHV}$. 
(ii) Adopt the notation of Remarks 3.2. The map $\phi: \mathbb{C}[V] \rightarrow \mathbb{C}\left[V^{*}\right]$ defined in (3.2) yields a bijective $\mathbb{C}$-antilinear morphism $\mathbb{C}[V]^{G}=\mathbb{C}[V]^{K} \rightarrow \mathbb{C}\left[V^{*}\right]^{K}=\mathbb{C}\left[V^{*}\right]^{G}$. Thus $\mathbb{C}\left[V^{*}\right]^{G}=\mathbb{C}\left[f^{*}\right]$.

(iii) Choose $v \in V$ regular semisimple, i.e. G.v closed and $\operatorname{dim} G . v \geq \operatorname{dim} G$. $v^{\prime}$ for all closed orbits $G \cdot v^{\prime}$. Set $\mathfrak{h}=\mathbb{C} v$ and $\mathfrak{g}=\operatorname{Lie}(G)$. Then, see [5], one easily deduces that $\mathfrak{h}=\mathfrak{h}_{v}=\{x \in V: \mathfrak{g} \cdot x \subset \mathfrak{g} \cdot v\}$ is a Cartan subspace for the $G$-action on $V$.

From now on, we assume that the hypothesis $A$ is satisfied and we fix a Cartan subspace $\mathfrak{h}=\mathbb{C} v$ for the $G$-action on $V$. We set

- $x=v^{*}$, hence $\mathbb{C}[\mathfrak{h}]=S\left(\mathfrak{h}^{*}\right)=\mathbb{C}[x]$;

- $W=N_{G}(\mathfrak{h}) / Z_{G}(\mathfrak{h})$.

By [5] we know that there exists an isomorphism $V / / G \cong \mathfrak{h} / W$ given by the restriction map $\psi: \mathbb{C}[V]^{G} \stackrel{\sim}{\longrightarrow}[\mathfrak{h}]^{W}, \psi(p(f))=p(f)_{\mid \mathfrak{h}}$. Since $f$ is homogeneous of degree $n, \psi(f) \in \mathbb{C}[x]$ is a scalar multiple of $z=x^{n}$. Therefore, multiplying $v$ by a non zero constant, we may assume that

$$
\psi(f)=x^{n}, \quad W \equiv\langle w\rangle \subset \mathrm{GL}(\mathfrak{h})
$$

where $w$ acts on $x$ by $w \cdot x=\zeta x, \zeta$ primitive $n$-th root of unity. We can therefore adopt the notation of $\S 2.2$. In particular, $\mathbb{C}[\mathfrak{h}]^{W}=\mathbb{C}[z]$ where $z=x^{n}=\psi(f)$.

Let $b(s)=c \prod_{i=0}^{n-1}\left(s+\lambda_{i}+1\right) \in \mathbb{C}[s]$ be a $b$-function of the relative invariant $f$ as in Theorem 3.1. We can then define the rational Cherednik algebra

$$
\mathcal{H}=\mathcal{H}\left(W, k_{0}, \ldots, k_{n-1}\right)=\mathbb{C}\langle x, T, w\rangle,
$$

where the parameters $k_{i}$ are given by $k_{i}=\lambda_{i}+\frac{i}{n}$, cf. (2.5). Recall that the image $U=\operatorname{res}(\mathrm{e} \mathcal{H} \mathrm{e}) \subset \mathbb{C}\left[z, \partial_{z}\right]$ of the spherical subalgebra is generated by $z, \delta, \theta$, where

$$
\theta=z \partial_{z}, \quad \delta=z^{-1} b^{*}(\theta)=z^{-1} c \prod_{j=0}^{n-1}\left(\theta+\lambda_{j}\right)
$$

see Proposition 2.5.

Recall from $\S 1$ that we have a radial component map:

$$
\operatorname{rad}: \mathcal{D}(V)^{G} \longrightarrow \mathcal{D}(\mathfrak{h} / W)=\mathbb{C}\left[z, \partial_{z}\right], \quad \operatorname{rad}(D)(p(z))=\psi(D(p(f))),
$$

for all $p(z) \in \mathbb{C}[\mathfrak{h}]^{W}$. The algebra of radial components is defined to be

$$
R=\operatorname{rad}\left(\mathcal{D}(V)^{G}\right) \subset \mathbb{C}\left[z, \partial_{z}\right] .
$$

The aim of this section is to prove that $R=U$, see Theorem 3.9.

Before entering the proof, let us give some standard examples. A complete list of pairs $(\tilde{G}: V)$ as above with $V$ irreducible can be found in [25]. Recall that $\Delta=f^{*}(\partial) \in S(V)$ is the differential operator constructed in (3.5).

Examples. (1) $\left(\tilde{G}=\mathrm{GL}(n): V=S^{2} \mathbb{C}^{n}\right), W=\mathbb{Z}_{n}, f=\operatorname{det}\left(x_{i j}\right), \Delta=\operatorname{det}\left(\partial_{x_{i j}}\right)$, $b(s)=\prod_{i=0}^{n-1}(s+i / 2+1)$.

(2) $\left(\tilde{G}=\mathrm{E}_{6} \times \mathbb{C}^{*}: V=\mathbb{C}^{27}\right), W=\mathbb{Z}_{3}, f=$ cubic form, $b(s)=(s+1)(s+5)(s+9)$.

(3) $\left(\tilde{G}=\operatorname{SO}(n) \times \mathbb{C}^{*}: V=\mathbb{C}^{n}\right), W=\mathbb{Z}_{2}, f=$ quadratic form, $\Delta=$ Laplacian, $b(s)=(s+1)(s+n / 2)$.

(4) $\left(\tilde{G}=\operatorname{SL}(5) \times \operatorname{GL}(4): V=\bigwedge^{2} \mathbb{C}^{5} \otimes \mathbb{C}^{4}\right), W=\mathbb{Z}_{40}, \operatorname{deg} f=40, b(s)$ is:

$$
\begin{gathered}
(s+1)^{8}[(s+2 / 3)(s+4 / 3)(s+3 / 4)(s+5 / 4)(s+5 / 6)(s+7 / 6)]^{4} \\
{[(s+7 / 10)(s+9 / 10)(s+11 / 10)(s+13 / 10)]^{2} .}
\end{gathered}
$$

(5) $\left(\tilde{G}=\mathrm{GL}(n) \times \mathrm{SL}(n): V=\mathrm{M}_{n}(\mathbb{C})\right), W=\mathbb{Z}_{n}, f=\operatorname{det}\left(x_{i j}\right), \Delta=\operatorname{det}\left(\partial_{x_{i j}}\right)$, $b(s)=\prod_{i=0}^{n-1}(s+i+1)$. 
(6) $\left(\tilde{G}=\operatorname{Sp}(n) \times \mathrm{SO}(3) \times \mathbb{C}^{*}: V=\mathrm{M}_{2 n, 3}(\mathbb{C})\right), W=\mathbb{Z}_{4}, \operatorname{deg} f=4, b(s)=$ $(s+1)(s+3 / 2)(s+n)(s+n+1 / 2)$.

Remark: The first five examples are regular irreducible PHV, but (6) gives is an example of an irreducible PHV which is not regular [25]. The description of the Verma modules on $U$ associated to examples (1) to (4) are given in $§ 2.3$.

Let $\Theta$ be the Euler vector field on $V$; thus $\Theta(p)=n p$ for all $p \in S^{n}\left(V^{*}\right)$. In particular $\Theta(f)=n f$, which implies that $\operatorname{rad}(\Theta)=n \theta$. Set

$$
\bar{\Theta}=\frac{1}{n} \Theta,
$$

so that $\operatorname{rad}(\bar{\Theta})=\theta$.

Lemma 3.4. One has:

$$
U \subset R, \quad U\left[z^{-1}\right]=R\left[z^{-1}\right]=\mathbb{C}\left[z^{ \pm 1}, \partial_{z}\right] .
$$

Proof. Let $\Delta=f^{*}(\partial) \in S(V)$ be as in in (3.5). By definition and Theorem 3.1 we obtain: $\operatorname{rad}(\Delta)\left(z^{s+1}\right)=\psi\left(\Delta\left(f^{s+1}\right)\right)=b(s) z^{s}$. Hence $\operatorname{rad}(\Delta)=\delta$, see Proposition 2.5. From $\operatorname{rad}(f)=z$ and $\operatorname{rad}(\bar{\Theta})=\theta$ it follows that $U=\mathbb{C}[z, \delta, \theta] \subset R \subset$ $\mathbb{C}\left[z, \partial_{z}\right]$. Observe that $U\left[z^{-1}\right]=\mathbb{C}\left[z^{ \pm 1}, \delta, z^{-1} \theta=\partial_{z},\right]=\mathbb{C}\left[z^{ \pm 1}, \partial_{z}\right] \subset R\left[z^{-1}\right] \subset$ $\mathbb{C}\left[z^{ \pm 1}, \partial_{z}\right]$, thus $U\left[z^{-1}\right]=R\left[z^{-1}\right]=\mathbb{C}\left[z^{ \pm 1}, \partial_{z}\right]$.

Recall that $\phi: \mathbb{C}[V] \rightarrow \mathbb{C}\left[V^{*}\right], \phi(p)=p^{*}$, is the bijective $\tilde{K}$-equivariant $\mathbb{C}$ antilinear morphism defined in (3.2).

Lemma 3.5. The map $\phi$ extends to a $\tilde{K}$-equivariant $\mathbb{C}$-antilinear anti-automorphism of $\mathcal{D}(V)$, given by $\phi(p)=\partial\left(p^{*}\right)$. One has:

$$
\phi\left(\mathcal{D}(V)^{G}\right)=\mathcal{D}(V)^{G}, \quad \phi^{2}=\mathrm{id}, \quad \phi(f)=\Delta=\partial\left(f^{*}\right), \quad \phi(\bar{\Theta})=\bar{\Theta} .
$$

Proof. In the coordinate system $\left\{z_{i}, \partial_{z_{i}}\right\}_{1 \leq i \leq N}$ we have: $\phi\left(z_{i}\right)=\partial_{z_{i}}, \phi\left(\partial_{z_{i}}\right)=z_{i}$, $\phi(a)=\bar{a}$ for $a \in \mathbb{C}$. Since $\mathcal{D}(V)=\mathbb{C}\left[z_{i}, \partial_{z_{j}}: 1 \leq i, j \leq N\right]$ with relations $\left[\partial_{z_{j}}, z_{i}\right]=\delta_{i j}$, it is clear that $\phi$ extends to a $\mathbb{C}$-antilinear anti-automorphism of $\mathcal{D}(V)$ such that $\phi^{2}=$ id. By construction $\phi$ is $\tilde{K}$-equivariant, in particular $K$-equivariant if $K \subset \tilde{K}$ is a maximal compact subgroup of $G$. From $G=K_{\mathbb{C}}=K \exp (i \mathfrak{k})$ it follows that $\mathcal{D}(V)^{K}=\mathcal{D}(V)^{G}$, hence $\phi\left(\mathcal{D}(V)^{G}\right)=\mathcal{D}(V)^{G}$. The equality $\phi(f)=\Delta$ is obvious and $\phi(\bar{\Theta})=\bar{\Theta}$ is consequence of $\Theta=\sum_{i} z_{i} \partial_{z_{i}}$.

Recall that rad : $\mathcal{D}(V)^{G} \rightarrow R$; we now want to check that the anti-automorphism $\phi$ induces an anti-automorphism on $R$ such that $\phi(z)=\delta$. Denote by $J$ the kernel of rad, thus:

$$
J=\left\{D \in \mathcal{D}(V)^{G}: D\left(f^{m}\right)=0 \text { for all } m \in \mathbb{N}\right\} .
$$

Set

$$
\mathbb{D}=\mathcal{D}(V)^{G} \supset \widetilde{\mathbb{D}}=\mathcal{D}(V)^{\tilde{G}} .
$$

Since $\Theta \in \mathcal{D}(V)^{G}$ we can decompose $\mathbb{D}$ under the adjoint action of $\Theta$ :

$$
\mathbb{D}=\bigoplus_{p \in \mathbb{Z}} \mathbb{D}[p], \quad \mathbb{D}[p]=\{D \in \mathbb{D}:[\Theta, D]=p D\} .
$$

Proposition 3.6. One has $\phi(J)=J$.

Proof. As $\phi^{2}=$ id we need to show that $\phi(J) \subset J$. Since $J$ is an ideal of $\mathcal{D}(V)^{G}$ it decomposes under the adjoint action of $\Theta: J=\oplus_{p \in \mathbb{Z}} J[p], J[p]=J \cap \mathbb{D}[p]$. Thus we only need to check that $\phi(J[p]) \subset J$. In the previous coordinate system $\left\{z_{i}, \partial_{z_{i}}\right\}_{1 \leq i \leq N}$ we have:

$$
\mathbb{D}[p]=\sum_{|\mathbf{i}|-|j|=p} \mathbb{C} z^{\mathrm{i}} \partial^{j}
$$


We can write $D \in \mathbb{D}[p]$ in a unique way under the form

$$
D=D_{0}+\cdots+D_{t}, \quad D_{k}=\sum_{|j|=k-p}\left(\sum_{|\mathbf{i}|=k} a_{\mathrm{i}, \mathrm{j}} z^{\mathrm{i}}\right) \partial^{\mathrm{j}},
$$

(thus $D_{k}=0$ when $k<p$ ). If $D_{t} \neq 0$, we set $t=\operatorname{deg}_{z} D$.

Let $D=\sum_{|\mathrm{i}|-|\mathrm{j}|=p} a_{\mathrm{i}, \mathrm{j}} z^{\mathrm{i}} \partial^{\mathrm{j}}$ be in $J[p] \backslash\{0\}$. As $G$ acts linearly on $V$ we have $G . D_{k} \subset S^{k}\left(V^{*}\right) S^{k-p}(V)$. Since $D$ is $G$-invariant it follows that each $D_{k}$ is $G$-invariant. We have:

$$
\phi(D)=\sum_{k} \phi\left(D_{k}\right), \quad \phi\left(D_{k}\right)=\sum_{\{|\mathrm{i}|=k,|j|=k-p\}} \overline{a_{\mathrm{i}, \mathrm{j}}} z^{\mathrm{j}} \partial^{\mathrm{i}} .
$$

From the previous expression we get that $\phi\left(D_{k}\right)(1)=0$ when $k>0$, hence

$$
\phi(D)(1)=\phi\left(D_{0}\right)(1)=\phi\left(D_{0}\right)=\sum_{|\mathrm{j}|=-p} \overline{a_{0, \mathrm{j}}} z^{\mathrm{j}} .
$$

Assume that $D_{0}=\sum_{|\mathrm{j}|=-p} a_{0, \mathrm{j}} \partial^{\mathrm{j}} \in S^{p}(V)^{G}$ is non zero. From Proposition 3.3(ii) we can deduce that $p=-\ell n, \ell \geq 0, D_{0}=\alpha \Delta^{\ell}, \alpha \neq 0$. By hypothesis $D\left(f^{\ell}\right)=$ $\sum_{k} D_{k}\left(f^{\ell}\right)=0$. Note that, since $|j|=k+n \ell>n \ell$ implies $\partial^{j}\left(f^{\ell}\right)=0$, we have $D_{k}\left(f^{\ell}\right)=\sum_{\{|\mathrm{i}|=k,|j|=k+n \ell\}} a_{\mathrm{i}, \mathrm{j}} z^{\mathrm{i}} \partial^{\mathrm{j}}\left(f^{\ell}\right)=0$ if $k>0$. Thus $D_{0}\left(f^{\ell}\right)=D\left(f^{\ell}\right)=0$. If $\ell=0$ we get $D_{0}=\alpha=D_{0}(1)=D_{0}\left(f^{\ell}\right)=0$, contradiction. Therefore $\ell \geq 1$ and

$$
0=D\left(f^{\ell}\right)=D_{0}\left(f^{\ell}\right)=\alpha \Delta^{\ell}\left(f^{\ell}\right)=\alpha b(\ell-1) \cdots b(0) .
$$

It is easily seen that $\Delta^{\ell}\left(f^{\ell}\right) \neq 0$, see [25, Proof of Proposition 2.22] (this is equivalent to $b(j) \neq 0$ for all $j \in \mathbb{N})$, hence a contradiction. Thus: $\phi(D)(1)=\phi\left(D_{0}\right)=$ $D_{0}=0$.

We show that $\phi(D) \in J$ by induction on $t=\operatorname{deg}_{z} D$. (In the case $t=0$ one has $D=D_{0}=0$.) Since $\Delta \in S(V)^{G}$ and $J$ is an ideal, one has $[D, \Delta] \in J$. Observe that

$$
[D, \Delta]=\sum_{k}\left[D_{k}, \Delta\right], \quad\left[D_{k}, \Delta\right]=\sum_{|j|=k-p}\left(\sum_{|\mathrm{i}|=k} a_{\mathrm{i}, \mathrm{j}}\left[z^{\mathrm{i}}, \Delta\right]\right) \partial^{\mathrm{j}} .
$$

But $\Delta \in S(V)^{G}$ implies that $\operatorname{deg}_{z}\left[z^{\mathrm{i}}, \Delta\right]<k=|\mathrm{i}|$, hence $\operatorname{deg}_{z}[D, \Delta]<t=\operatorname{deg}_{z} D$. Then, by induction, $\phi([D, \Delta])=[\phi(\Delta), \phi(D)]=[f, \phi(D)] \in J$. If $m \geq 0$ we then have: $0=[f, \phi(D)]\left(f^{m}\right)=f \phi(D)\left(f^{m}\right)-\phi(D)\left(f^{m+1}\right)$. This implies, by induction on $m, \phi(D)\left(f^{m+1}\right)=f \phi(D)\left(f^{m}\right)=f^{m+1} \phi(D)(1)$. It follows from the previous paragraph that $\phi(D)\left(f^{m+1}\right)=\phi(D)(1)=0$, i.e. $\phi(D) \in J$.

Corollary 3.7. (1) There exists a $\mathbb{C}$-antilinear anti-automorphism $\phi: R \rightarrow R$ such that:

$$
\phi^{2}=\mathrm{id}, \quad \phi(z)=\delta, \quad \phi(\theta)=\theta, \quad \phi(U)=U .
$$

(2) One has $U\left[\delta^{-1}\right]=R\left[\delta^{-1}\right]$.

Proof. (1) Let $\phi: \mathcal{D}(V)^{G} \rightarrow \mathcal{D}(V)^{G}$ be as in Lemma 3.5. By Proposition 3.6 we can define $\phi: R \rightarrow R$ by setting

$$
\phi(\operatorname{rad}(D))=\operatorname{rad}(\phi(D)) .
$$

Indeed: if $\operatorname{rad}(D)=\operatorname{rad}\left(D^{\prime}\right)$ we get $D-D^{\prime} \in J=\operatorname{Ker}(\operatorname{rad})$, hence $\phi(D)-\phi\left(D^{\prime}\right) \in J$ and $\operatorname{rad}(\phi(D))=\operatorname{rad}\left(\phi\left(D^{\prime}\right)\right)$. The equality $\phi^{2}=$ id is clear; by definition and Lemma 3.5:

$$
\phi(z)=\operatorname{rad}(\phi(f))=\operatorname{rad}(\Delta)=\delta, \quad \phi(\theta)=\operatorname{rad}(\phi(\bar{\Theta}))=\operatorname{rad}(\bar{\Theta})=\theta .
$$

From $U=\mathbb{C}[z, \delta, \theta]$ we then deduce $\phi(U)=U$.

(2) Observe that $\operatorname{ad}(\phi(u))^{m}(r)=(-1)^{m} \phi\left(\operatorname{ad}(u)^{m}(r)\right)$ for all $u, r \in R$. Since $\operatorname{ad}(z)$ is a locally nilpotent operator in $R$, it follows that $\operatorname{ad}(\phi(z))=\operatorname{ad}(\delta)$ has the same property. We can therefore construct the $\mathbb{C}$-algebras $U\left[\delta^{-1}\right] \subset R\left[\delta^{-1}\right]$. 
Let $Q=\operatorname{Frac}(U)$ be the fraction field of the Noetherian domain $U$. By Lemma 3.4 we know that $Q=\mathbb{C}\left(z, \partial_{z}\right)=\operatorname{Frac}(R)$. It is easy to see that $\phi$ extends to $Q$ by $\phi\left(s^{-1} a\right)=\phi(a) \phi(s)^{-1}$ for all $a \in r, 0 \neq s \in R$. This gives a $\mathbb{C}$-antilinear anti-automorphism of $Q$. Then $\left.\phi\left(R\left[z^{-1}\right]\right)\right)=\phi\left(U\left[z^{-1}\right]\right)=\phi(U)\left[\delta^{-1}\right]=U\left[\delta^{-1}\right]$, which yields $U\left[\delta^{-1}\right]=\phi\left(R\left[z^{-1}\right]\right)=\phi(R)\left[\delta^{-1}\right]=R\left[\delta^{-1}\right]$, as desired.

Let $M$ be a module over a $\mathbb{C}$-algebra $A$, then the Gelfand-Kirillov of $M$ is denoted by $\operatorname{GKdim}_{A} M$ or simply $\operatorname{GKdim} M$, see [32].

Lemma 3.8. Let $r \in R$. Then:

$$
\operatorname{GKdim}_{U}(U+U r) / U \leq \operatorname{GKdim}_{U} U-2=0 .
$$

Proof. From Corollary 3.7 we deduce that there exists $\nu \in \mathbb{N}$ such that $z^{\nu} r \in U$ and $\delta^{\nu} r \in U$. Therefore the $U$-module $(U+U r) / U$ is a factor of $U /\left(U z^{\nu}+U \delta^{\nu}\right)$. There exists on $U \cong \widetilde{U} /(\Omega)$ (cf. Proposition 2.8) a finite dimensional filtration such that $\operatorname{gr}(U)$ is isomorphic to the commutative algebra $\mathbb{C}[X, Y, S] /\left(X Y-S^{n}\right)$, see $\S 2.2$ or $[52,38]$, where $\operatorname{gr}(z)=X, \operatorname{gr}(\delta)=Y$. It follows that the associated graded module of $U /\left(U z^{\nu}+U \delta^{\nu}\right)$ is a factor of $\operatorname{gr}(U) /\left(\operatorname{gr}(U) X^{\nu}+\operatorname{gr}(U) Y^{\nu}\right)$, which is finite dimensional. Hence the result.

We now can prove the main result of this section.

Theorem 3.9. One has $U=R$.

Proof. Endow $U$ with a filtration such that $\operatorname{gr}(U) \cong \mathbb{C}[X, Y, S] /\left(X Y-S^{n}\right)$ as in the proof of the previous lemma. Observe that $\mathbb{C}[X, Y, S] /\left(X Y-S^{n}\right)$ is commutative Gorenstein normal domain. By [3, Theorem 3.9] $U$ is Auslander-Gorenstein and by [55] $U$ is a maximal order. Recall that $Q=\operatorname{Frac}(U)$ and consider the following family of finitely generated $U$-modules $M$ :

$$
\mathcal{F}=\left\{U \subset M \subset Q: \operatorname{GKdim}_{U} M / U \leq \operatorname{GKdim}_{U} U-2\right\} .
$$

From [3, Theorem 1.14] we know that $\mathcal{F}$ contains a unique maximal element $\tilde{M}$. By Lemma 3.8 we have $U+U r \subset \tilde{M}$ for all $r \in R$; hence $R \subset \tilde{M}$. It follows that $R$ is finitely generated over $U$ with $Q=\operatorname{Frac}(U)=\operatorname{Frac}(R)$. Thus $U=R$, since $U$ is a maximal order.

Remark 3.10. Let $(G: V)$ be a representation of the connected reductive group $G$ such that $\operatorname{dim} V / / G=1$. If $\mathbb{C}[V]^{G}=\mathbb{C}[f]$ one can define $\Delta \in S(V)^{G}$ and the polynomial $b(s)=c \prod_{i=0}^{n-1}\left(s+\lambda_{i}+1\right)$ as in Theorem 3.1. Then, the proof of Theorem 3.9 can be repeated to show that $R=\operatorname{Im}(\mathrm{rad})=U(k)$ (where $k_{i}=\lambda_{i}+\frac{i}{n}$, $0 \leq i \leq n-1)$.

\section{Multiplicity FReE REPRESEntations}

4.1. Generalities. Let $(\tilde{G}: V)$ be a connected reductive group. Write $\tilde{G}=G C$, $C \cong\left(\mathbb{C}^{*}\right)^{c}$, as in $\S 3.1$. We adopt the following notation:

- the Lie algebra of an algebraic group is denoted by the corresponding gothic character;

- $T U$ is a Borel subgroup of $G, T$ being a maximal torus of $G$, hence $\tilde{T} U$ is a Borel subgroup of $\tilde{G}, \tilde{T}=T C$;

- $\mathrm{R}$ is the root system of $(\mathfrak{g}, \mathfrak{t}), \mathrm{B}=\left\{\alpha_{1}, \ldots, \alpha_{\ell}\right\}$ is a basis of $\mathrm{R}$ and $\mathrm{R}^{+}$is the set of associated positive roots;

- $\Lambda$ is the weight lattice of $(\mathfrak{g}, \mathfrak{t})$, thus $\Lambda=\mathbb{Z} \varpi_{1} \oplus \cdots \oplus \mathbb{Z} \varpi_{\ell}$ where $\left\langle\varpi_{i}, \alpha_{j}\right\rangle=$ $\delta_{i j} ; \Lambda^{+}=\mathbb{N} \varpi_{1} \oplus \cdots \oplus \mathbb{N} \varpi_{\ell}$ denotes the dominant weights;

- $\tilde{\Lambda}=\Lambda \oplus \mathrm{X}(C)$, with $\mathrm{X}(C) \cong \mathbb{Z}^{c} ; \tilde{\Lambda}^{+}=\Lambda^{+} \oplus \mathrm{X}(C)$; 
- if $\tilde{\lambda} \in \tilde{\Lambda}^{+}$, resp. $\lambda \in \Lambda^{+}$, we denote by $E(\tilde{\lambda})$, resp. $E(\lambda)$, an irreducible $\tilde{\mathfrak{g}}$-module, resp. $\mathfrak{g}$-module, with highest weight $\tilde{\lambda}$, resp. $\lambda$; the dual of $E(\tilde{\lambda})$ is isomorphic to $E\left(\tilde{\lambda}^{*}\right), \tilde{\lambda}^{*}=-w_{0}(\tilde{\lambda})$ where $w_{0}$ is the longest element of the Weyl group of $\mathrm{R}$ ( similarly for $\left.E(\lambda)^{*}\right)$.

We fix a finite dimensional representation $(\tilde{G}: V)$ of the reductive group $\tilde{G}$. Then the rational $\tilde{G}$-module $\mathbb{C}[V]=S\left(V^{*}\right)$ decomposes as

$$
\mathbb{C}[V] \cong \bigoplus_{\tilde{\lambda} \in \tilde{\Lambda}^{+}} E(\tilde{\lambda})^{m(\tilde{\lambda})}
$$

where $m(\tilde{\lambda}) \in \mathbb{N} \cup\{\infty\}$.

Definition 4.1. The representation $(\tilde{G}: V)$ is called multiplicity free (MF for short) if $m(\tilde{\lambda}) \leq 1$ for all $\tilde{\lambda}$. In this case

$$
\mathbb{C}[V]=\bigoplus_{\tilde{\lambda} \in \tilde{\Lambda}^{+}} V(\tilde{\lambda})^{m(\tilde{\lambda})}, \quad m(\tilde{\lambda})=0,1
$$

where $V(\tilde{\lambda}) \subset S^{d(\tilde{\lambda})}\left(V^{*}\right)$ is isomorphic to $E(\tilde{\lambda})$; if $m(\tilde{\lambda})=1, d(\tilde{\lambda})$ is called the degree of $\tilde{\lambda}$ in $\mathbb{C}[V]$.

Remark. The MF representations are classified [21, 1, 27]. We give in Appendix A the list of $(\tilde{G}: V)$ with $V$ irreducible (see [21]). For instance, the examples (1), (2), (3), (5) given in $§ 3.2$ are $\mathrm{MF}$.

From now on, let $(\tilde{G}: V)$ be a $M F$ representation. The following results can be found, for example, in $[1,18,21,26]$.

- Set $\tilde{\Gamma}=\{\tilde{\lambda}: m(\tilde{\lambda})=1\}$, then $\tilde{\Gamma}=\bigoplus_{i=0}^{r} \mathbb{N} \tilde{\lambda}_{i}$ where the $\tilde{\lambda}_{i}$ are linearly independent over $\mathbb{Q}$.

- The algebra of $U$-invariants $\mathbb{C}[V]^{U}=\mathbb{C}\left[h_{0}, \ldots, h_{r}\right]$ is a polynomial ring. If $\tilde{\gamma}=\sum_{i} m_{j} \tilde{\lambda}_{j} \in \tilde{\Gamma}$, one has $V(\tilde{\gamma})=U(\tilde{\mathfrak{g}}) \cdot h^{\tilde{\gamma}}$ where $h^{\tilde{\gamma}}=h_{0}^{m_{0}} \cdots h_{r}^{m_{r}}$ is a highest weight vector of $V(\tilde{\gamma})$. In particular: $h_{j}=h^{\tilde{\lambda}_{j}}, d(\tilde{\gamma})=\sum_{j} m_{j} d\left(\tilde{\lambda}_{j}\right)$.

- The representation $(\tilde{G}: V)$ is a prehomogeneous vector space. Let $f_{0}, \ldots, f_{m}$ be the basic relative invariants of this $\mathrm{PHV}$ and let $\chi_{j} \in \mathrm{X}(\tilde{G})=\mathrm{X}(C), 0 \leq j \leq m$, be their weights. After identification of $\mathrm{X}(C)$ with a subgroup of $\tilde{\Lambda}$ as above, one can number the $\tilde{\lambda}_{j}$ so that

$$
\tilde{\lambda}_{0} \equiv \chi_{0}, \ldots, \tilde{\lambda}_{m} \equiv \chi_{m}, \quad h_{0}=f_{0}, \ldots, h_{m}=f_{m},
$$

thus $V\left(\tilde{\lambda}_{j}\right)=V\left(\chi_{j}\right)$ is the one dimensional $\tilde{G}$-module $\mathbb{C} f_{j}$.

Let $p: \tilde{\Lambda}^{+}=\Lambda^{+} \oplus \mathrm{X}(C) \rightarrow \Lambda^{+}$be the natural projection. Set

$$
\tilde{\Gamma}=\Gamma_{0} \bigoplus \Gamma, \quad \Gamma_{0}=\bigoplus_{j=0}^{m} \mathbb{N} \tilde{\lambda}_{j}=\bigoplus_{j=0}^{m} \mathbb{N} \chi_{j}, \quad \Gamma=\bigoplus_{j=m+1}^{r} \mathbb{N} \tilde{\lambda}_{j} .
$$

Using the results above, the next lemma is easy to prove.

Lemma 4.2. One has:

(a) $\Gamma_{0}=\mathrm{X}(C) \cap \tilde{\Gamma}=\{\tilde{\gamma} \in \tilde{\Gamma}: \tilde{\gamma}(\mathfrak{t})=0\} ; p$ induces a bijection $\Gamma \stackrel{\sim}{\longrightarrow} p(\Gamma)$;

(b) let $\tilde{\gamma} \in \tilde{\Gamma}$, then the $G$-module $V(\tilde{\gamma})$ is isomorphic to $E(p(\tilde{\gamma}))$;

(c) let $\gamma, \gamma^{\prime} \in \Gamma$, then the following are equivalent:

(i) $\gamma=\gamma^{\prime}$

(ii) $p(\gamma)=p\left(\gamma^{\prime}\right)$

(iii) $V(\gamma) \cong V\left(\gamma^{\prime}\right)$ as $G$-modules; 
(d) the algebra $\mathbb{C}[V]^{G}$ of $G$-invariants is polynomial ring, more precisely:

$$
\mathbb{C}[V]^{G}=\mathbb{C}\left[f_{0}, \ldots, f_{m}\right]=\bigoplus_{\gamma \in \Gamma_{0}} \mathbb{C} h^{\gamma} .
$$

Set:

$$
H\left(V^{*}\right)=\bigoplus_{\gamma \in \Gamma} V(\gamma)
$$

Lemma 4.3. The multiplication map:

$$
\mathrm{m}: H\left(V^{*}\right) \otimes_{\mathbb{C}} S\left(V^{*}\right)^{G} \longrightarrow S\left(V^{*}\right)=\mathbb{C}[V]
$$

is an isomorphism of $G$-modules.

Proof. Let $\tilde{\gamma}=\gamma+\gamma_{0}, \gamma \in \Gamma, \gamma_{0} \in \Gamma_{0}$. Observe that $C$ acts by scalars on the simple $\tilde{G}$-module $V(\tilde{\gamma})$; thus, since $\tilde{\mathfrak{g}}=\mathfrak{g} \oplus \mathfrak{c}$, we have: $V(\tilde{\gamma})=U(\tilde{\mathfrak{g}}) \cdot h^{\tilde{\gamma}}=U(\mathfrak{g}) U(\mathfrak{c}) \cdot h^{\tilde{\gamma}}=$ $U(\mathfrak{g}) \cdot h^{\tilde{\gamma}}=U(\mathfrak{g}) \cdot h^{\gamma} h^{\gamma_{0}}=\left(U(\tilde{\mathfrak{g}}) \cdot h^{\gamma}\right) h^{\gamma_{0}}=V(\gamma) h^{\gamma_{0}}$. Therefore $V(\tilde{\gamma})=\mathrm{m}(V(\gamma) \otimes$ $\left.\mathbb{C} h^{\gamma_{0}}\right)$ with $V(\gamma) \subset H\left(V^{*}\right), h^{\gamma_{0}} \in S\left(V^{*}\right)^{G}$. Suppose that $\mathrm{m}\left(\sum_{i} v_{i} \otimes h^{\mu_{i}}\right)=0$ with $\mu_{i} \in \Gamma_{0}, v_{i} \in V\left(\lambda_{i}\right)$, the $\lambda_{i} \in \Gamma$ being pairwise distinct. Observe that $\lambda_{i}+\mu_{i}=$ $\lambda_{j}+\mu_{j}$ forces $\lambda_{i}-\lambda_{j}=\mu_{j}-\mu_{i} \in \Gamma \cap \Gamma_{0}=(0)$. Therefore $v_{i} h^{\mu_{i}} \in V\left(\lambda_{i}+\mu_{i}\right)$ and $\sum_{i} v_{i} h^{\mu_{i}}=0$ yield $v_{i} h^{\mu_{i}}=0$, hence $v_{i}=0$ for all $i$.

Recall that we identify $S(V)$ with the algebra of differential operators with constant coefficients. Consider the non-degenerate pairing

$$
S(V) \otimes S\left(V^{*}\right) \longrightarrow \mathbb{C}, \quad u \otimes \varphi \mapsto\langle u \mid \varphi\rangle=u(\varphi)(0),
$$

which extends the duality pairing $V \otimes V^{*} \rightarrow \mathbb{C}$. It is easily shown that:

- $\left\langle u \mid S^{j}\left(V^{*}\right)\right\rangle=0$ if $u \in S^{i}(V)$ and $i \neq j$;

- $\langle\mid\rangle$ is $\tilde{G}$-equivariant.

Therefore $u \mapsto\langle u \mid\rangle$ gives a $\tilde{G}$-isomorphism from $S^{i}(V)$ onto $S^{i}\left(V^{*}\right)^{*}$. In particular, the representation $\left(\tilde{G}: V^{*}\right)$ is $\mathrm{MF}$ and we can write:

$$
S^{i}(V)=\bigoplus_{\{\tilde{\gamma} \in \tilde{\Gamma}, d(\tilde{\gamma})=i\}} Y(\tilde{\gamma}), \quad Y(\tilde{\gamma}) \cong V(\tilde{\gamma})^{*} \cong E\left(\tilde{\gamma}^{*}\right) .
$$

Hence, $Y(\tilde{\gamma})=U(\tilde{\mathfrak{g}}) \cdot \Delta^{\tilde{\gamma}}$ where $\Delta^{\tilde{\gamma}}$ is a lowest weight vector (of weight $-\tilde{\gamma}$ ). When $\tilde{\gamma}=\tilde{\lambda}_{j}$ we set $\Delta^{\tilde{\lambda}_{j}}=\Delta_{j}$. Note that $\Delta^{\tilde{\gamma}}=\prod_{i=0}^{r} \Delta_{i}^{m_{i}}$ if $\tilde{\gamma}=\sum_{i} m_{i} \tilde{\lambda}_{i}$.

If $0 \leq i \leq m$ we have $Y\left(\tilde{\lambda}_{i}\right)=\mathbb{C} \Delta_{i}$ where $\Delta_{i}$ has weight $-\lambda_{i} \equiv \chi_{i}^{-1}$. Clearly, we may take $\Delta_{i}=\partial\left(f_{i}^{*}\right)$ where $f_{i}^{*}$ is the relative invariant constructed as in $\S 3.1$. We then have

$$
S(V)^{G}=\mathbb{C}\left[\Delta_{0}, \ldots, \Delta_{m}\right]=\bigoplus_{\gamma \in \Gamma_{0}} \mathbb{C} \Delta^{\gamma}
$$

(which is a polynomial ring).

If $\mu=\sum_{i} m_{i} \tilde{\lambda}_{i}$ and $\nu=\sum_{i} n_{i} \tilde{\lambda}_{i}$ are elements of $\tilde{\Gamma}$, we say that $\mu \leq \nu$ if $m_{i} \leq n_{i}$ for all $i$. Let $k: \tilde{\Gamma} \rightarrow \Gamma_{0}$ be the projection associated to the decomposition defined in (4.1); thus each $\tilde{\lambda} \in \tilde{\Lambda}$ writes uniquely $\gamma+k(\tilde{\lambda}), \gamma \in \Gamma, k(\tilde{\lambda}) \in \Gamma_{0}$.

Lemma 4.4. Let $\tilde{\lambda} \in \Gamma_{0}$ and $\tilde{\gamma} \in \tilde{\Gamma}$. Then:

(a) $\Delta^{\tilde{\gamma}}\left(h^{\tilde{\gamma}}\right) \neq 0$;

(b) $\Delta^{\tilde{\lambda}}\left(h^{\tilde{\gamma}}\right) \neq 0 \Longleftrightarrow \tilde{\lambda} \leq k(\tilde{\gamma})$, and in this case $\Delta^{\tilde{\lambda}}$ gives an isomorphism of $G$-modules, $\varphi \mapsto \Delta^{\tilde{\lambda}}(\varphi)$, from $V(\tilde{\gamma})$ onto $V(\tilde{\gamma}-\tilde{\lambda})$.

Proof. Set $\tilde{\lambda}=\sum_{i=0}^{m} p_{i} \tilde{\lambda}_{i}, \tilde{\gamma}=\sum_{i=0}^{r} q_{i} \tilde{\lambda}_{i}$.

(a) Recall that we have an isomorphism of $\tilde{G}$-modules, $\beta: Y(\tilde{\gamma}) \stackrel{\sim}{\longrightarrow} V(\tilde{\gamma})^{*}$, $\beta(u)=\langle u \mid\rangle$. Thus $\beta\left(\Delta^{\tilde{\gamma}}\right)$ is a lowest vector in $V(\tilde{\gamma})^{*}$, which implies $\beta\left(\Delta^{\tilde{\gamma}}\right)\left(h^{\tilde{\gamma}}\right)=$ 
$\Delta^{\tilde{\gamma}}\left(h^{\tilde{\gamma}}\right)(0) \neq 0$. But $\Delta^{\tilde{\gamma}} \in S^{d(\tilde{\gamma})}(V)$ where $d(\tilde{\gamma})$ is the degree of $\tilde{\gamma}$, therefore $\Delta^{\tilde{\gamma}}\left(h^{\tilde{\gamma}}\right) \in \mathbb{C}$. Thus $\Delta^{\tilde{\gamma}}\left(h^{\tilde{\gamma}}\right)=\Delta^{\tilde{\gamma}}\left(h^{\tilde{\gamma}}\right)(0) \neq 0$.

(b) Since $\Delta^{\tilde{\lambda}} \in S(V)^{G}$ we have $\Delta^{\tilde{\lambda}}(V(\tilde{\gamma}))=\Delta^{\tilde{\lambda}}\left(U(\mathfrak{g}) h^{\tilde{\gamma}}\right)=U(\mathfrak{g}) \Delta^{\tilde{\lambda}}\left(h^{\tilde{\gamma}}\right)$. By Lemma 4.2 we know that $V(\tilde{\gamma})$ is a simple $G$-module, it follows that the map $\Delta^{\tilde{\lambda}}: V(\tilde{\gamma}) \rightarrow \Delta^{\tilde{\lambda}}(V(\tilde{\gamma}))$ is either 0 or an isomorphism of $G$-modules.

Notice that $\Delta^{\tilde{\lambda}}\left(h^{\tilde{\gamma}}\right) \in \mathbb{C}[V]^{U}$ has weight $\tilde{\gamma}-\tilde{\lambda}=k(\tilde{\gamma})-\tilde{\lambda}+\sum_{i=m+1}^{r} q_{i} \tilde{\lambda}_{i}$ where $k(\tilde{\gamma})-\tilde{\lambda}=\sum_{i=0}^{m}\left(q_{i}-p_{i}\right) \tilde{\lambda}_{i}$. Therefore if $q_{i}<p_{i}$ for some $i=0, \ldots, m$ we must have $\Delta^{\tilde{\lambda}}\left(h^{\tilde{\gamma}}\right)=0$, i.e. $\Delta^{\tilde{\lambda}}\left(h^{\tilde{\gamma}}\right) \neq 0$ implies $\tilde{\lambda} \leq k(\tilde{\gamma})$. Conversely, suppose that $\tilde{\lambda} \leq k(\tilde{\gamma})$; then, by (a),

$$
0 \neq \Delta^{\tilde{\gamma}}\left(h^{\tilde{\gamma}}\right)=\Delta^{\tilde{\gamma}-\tilde{\lambda}} \Delta^{\tilde{\lambda}}\left(h^{\tilde{\gamma}}\right)
$$

which forces $\Delta^{\tilde{\lambda}}\left(h^{\tilde{\gamma}}\right) \neq 0$.

Now assume $\tilde{\lambda} \leq k(\tilde{\gamma})$. Then $0 \neq \Delta^{\tilde{\lambda}}\left(h^{\tilde{\gamma}}\right) \in \mathbb{C}[V]^{U}$ implies that $\Delta^{\tilde{\lambda}}\left(h^{\tilde{\gamma}}\right)$ is a highest weight vector in $V(\tilde{\gamma}-\tilde{\lambda})$, hence $\Delta^{\tilde{\lambda}}: V(\tilde{\gamma}) \stackrel{\sim}{\longrightarrow} \Delta^{\tilde{\lambda}}(V(\tilde{\gamma}))=V(\tilde{\gamma}-\tilde{\lambda})$.

Recall the definition of $H\left(V^{*}\right)$ given in (4.2) and set $S_{+}(V)=\bigoplus_{i>0} S^{i}(V)$. The next proposition identifies $H\left(V^{*}\right)$ with harmonic elements.

Proposition 4.5. We have:

$$
\begin{aligned}
H\left(V^{*}\right) & =\left\{\varphi \in \mathbb{C}[V]: \Delta_{0}(\varphi)=\cdots=\Delta_{m}(\varphi)=0\right\} \\
& =\left\{\varphi \in \mathbb{C}[V]: D(\varphi)=0 \text { for all } D \in S_{+}(V)^{G}\right\} .
\end{aligned}
$$

Proof. From (4.3) we know that $S_{+}(V)^{G}=\bigoplus_{0 \neq \tilde{\lambda} \in \Gamma_{0}} \mathbb{C} \Delta^{\tilde{\lambda}}$. Let $\varphi \in V(\tilde{\gamma})$ for some $\tilde{\gamma} \in \Gamma$ and let $0 \neq \tilde{\lambda} \in \Gamma_{0}$. We have $k(\tilde{\gamma})=0$, thus $\Delta^{\tilde{\lambda}}(\varphi)=0$ by Lemma $4.4(\mathrm{~b})$. This shows that $H\left(V^{*}\right) \subset\left\{\varphi \in \mathbb{C}[V]: D(\varphi)=0\right.$ for all $\left.D \in S_{+}(V)^{G}\right\}$.

Conversely assume that $\varphi=\sum_{\tilde{\gamma} \in \tilde{\Gamma}} \varphi_{\tilde{\gamma}}, \varphi_{\tilde{\gamma}} \in V(\tilde{\gamma})$, satisfies $\Delta_{i}(\varphi)=0$ for all $i=0, \ldots, m$. Fix $i \in\{0, \ldots, m\}$. By Lemma $4.4(\mathrm{~b})$ we get that $\Delta_{i}(V(\tilde{\gamma}))=$ 0 if $\tilde{\lambda}_{i} \not \leq k(\tilde{\gamma})$ and $\Delta_{i}: V(\tilde{\gamma}) \stackrel{\sim}{\longrightarrow} V\left(\tilde{\gamma}-\tilde{\lambda}_{i}\right)$ if $\tilde{\lambda}_{i} \leq k(\tilde{\gamma})$. Therefore $\Delta_{i}(\varphi)=$ $\sum_{\tilde{\gamma} \in \tilde{\Gamma}} \Delta_{i}\left(\varphi_{\tilde{\gamma}}\right)$ belongs to $\bigoplus_{\left\{\tilde{\gamma} \in \tilde{\Gamma}, \tilde{\lambda}_{i} \leq k(\tilde{\gamma})\right\}} V\left(\tilde{\gamma}-\tilde{\lambda}_{i}\right)$. Since $\Delta_{i}(\varphi)=0$ we can deduce that $\Delta_{i}\left(\varphi_{\tilde{\gamma}}\right)=0$ for all $\tilde{\gamma}$ such that $\tilde{\lambda}_{i} \leq k(\tilde{\gamma})$. By the previous remark this implies $\varphi_{\tilde{\gamma}}=0$ when $\tilde{\lambda}_{i} \leq k(\tilde{\gamma})$, thus $\varphi=\sum_{\left\{\tilde{\gamma} \in \tilde{\Gamma}, \tilde{\lambda}_{i} \not k(\tilde{\gamma})\right\}} \varphi_{\tilde{\gamma}}$. Observe that $\tilde{\lambda}_{i} \not \leq k(\tilde{\gamma})$ means that the weight $\tilde{\lambda}_{i}$ does not appear in $\tilde{\gamma}$. Since this holds for all $i=0, \ldots, m$ we deduce that $\varphi=\sum_{\tilde{\gamma} \in \Gamma} \varphi_{\tilde{\gamma}}$. Hence the result.

Remark 4.6. If we set $H(V)=\bigoplus_{\gamma \in \Gamma} Y(\gamma)$ we obtain that $S(V) \cong H(V) \otimes S(V)^{G}$ as $G$-modules, with an analogous characterization of $H(V)$.

We now recall some facts about invariant differential operators on MF representations, cf. $[1,18,26]$. Recall that the $\mathbb{C}[V]$-module $\mathcal{D}(V)$ identifies with $S\left(V^{*}\right) \otimes S(V)$ through the multiplication map

$$
\mathrm{m}: S\left(V^{*}\right) \otimes S(V) \stackrel{\sim}{\longrightarrow} \mathcal{D}(V), \quad \varphi \otimes f \mapsto \varphi f(\partial) .
$$

The isomorphism $\mathrm{m}$ is also $\tilde{G}$-equivariant, hence $\mathcal{D}(V)^{\tilde{G}} \cong \bigoplus_{\tilde{\gamma} \in \tilde{\Gamma}}[V(\tilde{\gamma}) \otimes Y(\tilde{\gamma})]^{\tilde{G}}$. But, since $Y(\tilde{\gamma}) \cong V(\tilde{\gamma})^{*},[V(\tilde{\gamma}) \otimes Y(\tilde{\gamma})]^{\tilde{G}}=\mathbb{C} E_{\tilde{\gamma}}$ is one dimensional. Let

$$
E_{\tilde{\gamma}}\left(x, \partial_{x}\right)=\frac{1}{\operatorname{dim} V(\tilde{\gamma})} \mathrm{m}\left(E_{\tilde{\gamma}}\right) \in \mathcal{D}(V)^{\tilde{G}}
$$

be the operator corresponding to $E_{\tilde{\gamma}}$. The $E_{\tilde{\gamma}}\left(x, \partial_{x}\right)$ are called the normalized Capelli operators. Set

$$
E_{j}=E_{\tilde{\lambda}_{j}}\left(x, \partial_{x}\right), \quad 0 \leq j \leq r .
$$


It is known [18, Proposition 7.1] that $(\tilde{G}: V)$ multiplicity free is equivalent to $\mathcal{D}(V)^{\tilde{G}}$ commutative. The operators $E_{j}$ give a set of generators for this algebra, cf. [18, Theorem 9.1] or [1, Corollary 7.4.4]:

Theorem 4.7 (Howe-Umeda). $\widetilde{\mathbb{D}}=\mathcal{D}(V)^{\tilde{G}}=\mathbb{C}\left[E_{0}, \ldots, E_{r}\right]=\bigoplus_{\tilde{\gamma} \in \tilde{\Gamma}} \mathbb{C} E_{\tilde{\gamma}}\left(x, \partial_{x}\right)$ is a commutative polynomial ring.

Notice for further use the following property of the Capelli operators, see [26, Corollary 4.4] or [1, Proposition 8.3.2]:

Proposition 4.8. Set $\mathfrak{a}^{*}=\mathbb{C} \otimes_{\mathbb{Z}} \mathbb{Z} \tilde{\Gamma}=\bigoplus_{i=0}^{r} \mathbb{C} \tilde{\lambda}_{i}$, a $=\bigoplus_{i=0}^{r} \mathbb{C} a_{i}$ where $\left\{a_{i}\right\}_{i}$ is the dual basis of $\left\{\tilde{\lambda}_{i}\right\}_{i}$. For each $\tilde{\gamma} \in \tilde{\Gamma}$ there exists a polynomial function $b_{\tilde{\gamma}}=$ $b_{\tilde{\gamma}}\left(a_{0}, \ldots, a_{r}\right) \in \mathbb{C}\left[\mathfrak{a}^{*}\right]=S(\mathfrak{a})=\mathbb{C}\left[a_{0}, \ldots, a_{r}\right]$ such that

$$
E_{\tilde{\gamma}}\left(x, \partial_{x}\right)\left(h^{\tilde{\lambda}}\right)=b_{\tilde{\gamma}}(\tilde{\lambda}) h^{\tilde{\lambda}} \text { for all } \tilde{\lambda} \in \mathfrak{a}^{*} .
$$

Remarks 4.9. (1) Suppose that $(\tilde{G}: V)$ is irreducible. Then we can assume that $V=V\left(\tilde{\gamma}_{r}\right)$. If $\operatorname{dim} V=N$ we have $E_{r}=E_{r}\left(x, \partial_{x}\right)=\bar{\Theta}=\frac{1}{N} \Theta$ where $\Theta$ is the Euler vector field.

(2) If $j \in\{0, \ldots, m\}$ we may take $E_{j}=f_{j} \Delta_{j}$. Recall that $f_{j}=h^{\tilde{\lambda}_{j}}$ and $\Delta_{j}=$ $\partial\left(f_{j}^{*}\right)$. By Theorem 3.1 there exists $b_{j}(s) \in \mathbb{C}[s]$ such that $\Delta_{j}\left(f_{j}^{m}\right)=b_{j}^{*}(m) f_{j}^{m-1}$; thus $E_{j}\left(f_{j}^{m}\right)=b_{j}^{*}(m) f_{j}^{m}$. This shows that $b_{\tilde{\lambda}_{j}}(s, 0, \ldots, 0)=b_{j}^{*}(s)$.

(3) Let $D \in \widetilde{\mathbb{D}}$; then $D(V(\tilde{\lambda}))=U(\tilde{\mathfrak{g}}) \cdot D\left(h^{\tilde{\lambda}}\right)$ is either $(0)$ or equal to $V(\tilde{\lambda})$. Indeed, the $\tilde{G}$-invariance of $D$ implies that $g \cdot D\left(h^{\tilde{\lambda}}\right)=D\left(g \cdot h^{\tilde{\lambda}}\right)=\tilde{\lambda}(g) D\left(h^{\tilde{\lambda}}\right)$ for all $g \in \tilde{T} U$, where we have considered here $\tilde{\lambda}$ as a character of the Borel subgroup $\tilde{T} U$ of $\tilde{G}$; thus $D\left(h^{\tilde{\lambda}}\right) \in \mathbb{C} h^{\tilde{\lambda}}$ is either 0 or a highest weight vector of $V(\tilde{\lambda})$.

4.2. MF representations with a one dimensional quotient. In this subsection we will work under the following hypothesis:

Hypothesis B. $(\tilde{G}: V)$ is a multiplicity free representation which satisfies $H y$ pothesis $A$.

In the notation of $\S 4.1$, this condition means that $m=0$, i.e. $\Gamma_{0}=\mathbb{N} \tilde{\lambda}_{0}$. Set $f=f_{0}, n=d\left(\tilde{\lambda}_{0}\right), \Delta=\Delta_{0}=\partial\left(f^{*}\right)$, then we have $f \in S^{n}\left(V^{*}\right), V\left(\tilde{\lambda}_{0}\right)=\mathbb{C} f$, $Y\left(\tilde{\lambda}_{0}\right)=\mathbb{C} \Delta$ and:

$$
\mathbb{C}[V]^{G}=\mathbb{C}[f], \quad S(V)^{G}=\mathbb{C}[\Delta]
$$

(see Lemma 4.2 and (4.3)). By Remark 4.9(2) we have $E_{0}=f \Delta, b_{\tilde{\lambda}_{j}}(s, 0, \ldots, 0)=$ $b^{*}(s)=b(s-1)$ where $b(s)$ is the $b$-function of $f$. Recall from (3.8) the following notation:

$$
\mathbb{D}=\mathcal{D}(V)^{G} \supset \mathcal{D}(V)^{\tilde{G}}=\widetilde{\mathbb{D}} .
$$

Recall also that $(G: V)$ is polar and that we have studied in $\S 3.2$ the image of radial component map $\operatorname{rad}: \mathcal{D}(V)^{G} \rightarrow \mathcal{D}(\mathfrak{h} / W)=\mathbb{C}\left[z, \partial_{z}\right]$. We now want to describe $J=\operatorname{Ker}(\operatorname{rad})$.

Lemma 4.10. Let $P \in \widetilde{\mathbb{D}}$. Then there exists a polynomial $b_{P}(s) \in \mathbb{C}[s]$ such that

$$
P\left(f^{m}\right)=b_{P}(m) f^{m}, \quad \operatorname{rad}(P)=b_{P}(\theta), \quad P-b_{P}(\bar{\Theta}) \in J=\operatorname{Ker}(\operatorname{rad}) .
$$

Proof. Write $P=\sum_{\gamma \in \tilde{\Gamma}} p_{\gamma} E_{\tilde{\gamma}}\left(x, \partial_{x}\right)$, cf. Theorem 4.7, and define a polynomial function by $b_{P}(s)=\sum_{\tilde{\gamma} \in \tilde{\Gamma}} p_{\tilde{\gamma}} b_{\tilde{\gamma}}(s, 0, \ldots, 0)$, where $b_{\tilde{\gamma}} \in S(\mathfrak{a})$ is as in Proposition 4.8. Since $f^{m}=h^{m \tilde{\lambda}_{0}}$ we obtain that $P\left(f^{m}\right)=b_{P}(m) f^{m}$. It follows that $\operatorname{rad}(P)\left(z^{m}\right)=$ $b_{P}(m) z^{m}$ for all $m \in \mathbb{N}$ and Lemma 2.1 yields $\operatorname{rad}(P)=b_{P}(\theta)$. Since $\operatorname{rad}(\bar{\Theta})=\theta$ we have $\operatorname{rad}\left(P-b_{P}(\bar{\Theta})\right)=0$. 
Notice that $\bar{\Theta} \in \widetilde{\mathbb{D}}$; for $j \in\{0, \ldots, r\}$ we set

$$
\Omega_{j}=E_{j}-b_{E_{j}}(\bar{\Theta}) \in J \cap \widetilde{\mathbb{D}} .
$$

Thus we have:

$$
\widetilde{\mathbb{D}}=\mathbb{C}\left[E_{0}, \ldots, E_{r}\right]=\mathbb{C}\left[\Omega_{0}, \Omega_{1}, \ldots, \Omega_{r}, \bar{\Theta}\right] .
$$

Recall that for $j=0$ one has $E_{0}=f \Delta$, hence $b_{E_{0}}(s)=b^{*}(s)$ where $b(s)$ is the $b$-function of $f$. Thus $\Omega_{0}=f \Delta-b^{*}(\bar{\Theta})$; observe that we have already shown in $\S 3.2$ that $\operatorname{rad}\left(f \Delta-b^{*}(\bar{\Theta})\right)=z \delta-b^{*}(\theta)=0$.

When $V$ is irreducible we adopt the notation of Remark 4.9(1) and we obtain $E_{r}=\bar{\Theta}, b_{E_{r}}(s)=s$, thus $\Omega_{r}=0$. Therefore in this case one has

$$
\widetilde{\mathbb{D}}=\mathbb{C}\left[\bar{\Theta}, \Omega_{0}, \ldots, \Omega_{r-1}\right] .
$$

The next result gives a description of $\operatorname{Ker}(\mathrm{rad})$ and another proof of Theorem 3.9 in the case of MF representations. When $(\tilde{G}: V)=\left(\operatorname{GL}(n): S^{2} \mathbb{C}^{n}\right)$, part (i) of Theorem 4.11 is proved in [36, Proposition 2.1].

Theorem 4.11. The following properties hold.

(i) $\mathbb{D}=\widetilde{\mathbb{D}}[f, \Delta]=\mathbb{C}\left[E_{1}, \ldots, E_{r}\right][f, \Delta]=\mathbb{C}\left[\Omega_{1}, \ldots, \Omega_{r}\right][f, \Delta, \bar{\Theta}]$.

(ii) $\mathbb{D}=\left(\bigoplus_{p \in \mathbb{N}} \widetilde{\mathbb{D}} f^{p}\right) \oplus\left(\bigoplus_{p \in \mathbb{N}^{*}} \widetilde{\mathbb{D}} \Delta^{p}\right)$.

(iii) For $k \in \mathbb{Z}$, set

$$
\mathbb{D}[k]= \begin{cases}\widetilde{\mathbb{D}} f^{k} & \text { if } k \geq 0, \\ \widetilde{\mathbb{D}} \Delta^{-k} & \text { if } k<0 ;\end{cases}
$$

then $\mathbb{D}[k]=f^{k} \widetilde{\mathbb{D}}$, ik $k \geq 0$, or $\Delta^{-k} \widetilde{\mathbb{D}}$, if $k<0$.

(iv) $R=\operatorname{rad}(\mathbb{D})=U=\mathbb{C}[z, \delta, \theta]$.

(v) $J=\operatorname{Ker}(\mathrm{rad})=\sum_{i=0}^{r} \mathbb{D} \Omega_{i}=\sum_{i=0}^{r} \Omega_{i} \mathbb{D}$.

Proof. Endow $\mathcal{D}(V), \mathbb{D}$ and $\widetilde{\mathbb{D}}$ with the "Bernstein filtration", i.e.:

$$
\mathcal{F}_{p} \mathcal{D}(V)=\sum_{i+j \leq p} S^{i}\left(V^{*}\right) S^{j}(V), \quad \mathcal{F}_{p} \mathbb{D}=\mathcal{F}_{p} \mathcal{D}(V) \cap \mathbb{D} \supset \mathcal{F}_{p} \widetilde{\mathbb{D}}=\mathcal{F}_{p} \mathcal{D}(V) \cap \widetilde{\mathbb{D}}
$$

Then, since $\tilde{G}$ and $G$ are reductive,

$$
\widetilde{\mathbb{S}}=\left[\operatorname{gr}_{\mathcal{F}} \mathcal{D}(V)\right]^{\tilde{G}}=\left[S\left(V^{*}\right) \otimes S(V)\right]^{\tilde{G}}, \quad \mathbb{S}=\left[\operatorname{gr}_{\mathcal{F}} \mathcal{D}(V)\right]^{G}=\left[S\left(V^{*}\right) \otimes S(V)\right]^{G} .
$$

Denote by $\sigma_{j}=\operatorname{gr}_{\mathcal{F}}\left(E_{j}\right) \in\left[V\left(\tilde{\lambda}_{j}\right) \otimes Y\left(\tilde{\lambda}_{j}\right)\right]^{\tilde{G}}$ the principal symbol of $E_{j}$ for $\mathcal{F}$. Then $\widetilde{\mathbb{S}}=\mathbb{C}\left[\sigma_{0}, \ldots, \sigma_{r}\right]$, see for example [1]. Recall that $E_{0}=f \Delta$, hence $\sigma_{0}=f f^{*}$. By Lemma 4.3 and Remark 4.6 we know that $S\left(V^{*}\right)=H\left(V^{*}\right) \otimes \mathbb{C}[f], S(V)=H(V) \otimes$ $\mathbb{C}\left[f^{*}\right]$, hence $\mathbb{S}=\left[H\left(V^{*}\right) \otimes H(V)\right]^{G} \otimes \mathbb{C}\left[f, f^{*}\right]$ (as vector spaces). Let $\gamma, \lambda \in \Gamma$; recall that the $G$-module $V(\gamma)$ is isomorphic to $E(p(\gamma))$ and that $p(\gamma)=p(\lambda)$ if and only if $\gamma=\lambda$, cf. Lemma 4.2. It follows that $[V(\gamma) \otimes Y(\gamma)]^{G}=[V(\gamma) \otimes Y(\gamma)]^{\tilde{G}}=\mathbb{C} E_{\gamma}$ and

$$
\left[H\left(V^{*}\right) \otimes H(V)\right]^{G}=\bigoplus_{\gamma \in \Gamma} \mathbb{C} E_{\gamma} \subset \widetilde{\mathbb{S}}=\mathbb{C}\left[\sigma_{0}, \ldots, \sigma_{r}\right]
$$

Thus:

$$
\widetilde{\mathbb{S}}\left[f, f^{*}\right] \subset \mathbb{S}=\left[H\left(V^{*}\right) \otimes H(V)\right]^{G} \otimes \mathbb{C}\left[f, f^{*}\right] \subset \widetilde{\mathbb{S}}\left[f, f^{*}\right] .
$$

Since the centre $C$ of $\tilde{G}$ acts trivially on $\widetilde{\mathbb{S}}$ and via $\chi^{j}$, resp. $\chi^{-j}$, on $f^{j}$, resp. $\left(f^{*}\right)^{j}$, we obtain:

$$
\mathbb{S}=\widetilde{\mathbb{S}}\left[f, f^{*}\right]=\left(\bigoplus_{j \geq 0} \widetilde{\mathbb{S}} f^{i}\right) \oplus\left(\bigoplus_{i>0} \widetilde{\mathbb{S}}\left(f^{*}\right)^{i}\right)=\mathbb{C}\left[\sigma_{1}, \ldots, \sigma_{r}\right] \otimes \mathbb{C}\left[f, f^{*}\right]
$$


Then, by a filtration argument, one deduces that $\mathbb{D}=\sum_{p} \tilde{\mathbb{D}} f^{p}+\sum_{p} \widetilde{\mathbb{D}} \Delta^{p}=$ $\widetilde{\mathbb{D}}[f, \Delta]=\mathbb{C}\left[E_{1}, \ldots, E_{r}\right][f, \Delta]=\mathbb{C}\left[\Omega_{1}, \ldots, \Omega_{r}\right][f, \Delta, \bar{\Theta}]$ (recall that $\Omega_{j}=E_{j}-$ $\left.b_{E_{j}}(\bar{\Theta})\right)$. This proves (i).

Observe that $\widetilde{\mathbb{D}} f^{p}$ and $f^{p} \widetilde{\mathbb{D}}$, resp. $\widetilde{\mathbb{D}} \Delta^{p}$ and $\Delta^{p} \widetilde{\mathbb{D}}$, are contained in the $\chi^{p}$-weight space, resp. $\chi^{-p}$-weight space, for the action of $C$ on $\mathbb{D}$. This implies easily, as in $(\star)$, that these one dimensional subspaces are equal to the corresponding weight spaces. This proves (ii) and (iii).

Since $\Omega_{i} \in J=\operatorname{Ker}(\operatorname{rad})$, we obtain $\operatorname{rad}(\mathbb{D})=\mathbb{C}[\operatorname{rad}(f), \operatorname{rad}(\Delta), \operatorname{rad}(\bar{\Theta})]=$ $\mathbb{C}[z, \delta, \theta]$, hence (iv).

(v) Let $P \in \mathbb{D}$ and write $P=\sum_{k} P_{k}, P_{k} \in \mathbb{D}[k]$ with $P_{k}=Q_{k} f^{k}$ or $Q_{k}^{\prime} \Delta^{-k}$ and $Q_{k}, Q_{k}^{\prime} \in \widetilde{\mathbb{D}}=\mathbb{C}\left[\Omega_{0}, \ldots, \Omega_{r}, \bar{\Theta}\right]$. Set:

$$
Q_{p}=\sum_{i \geq 0} Q_{p, i} \bar{\Theta}^{i}, \quad Q_{p}=\sum_{i \geq 0} Q_{p, i}^{\prime} \bar{\Theta}^{i}
$$

where $Q_{p, i}, Q_{p, i}^{\prime} \in \mathbb{C}\left[\Omega_{0}, \ldots, \Omega_{r}\right]$. Since $Q_{p, i} \in Q_{p, i}(0)+\sum_{j} \Omega_{j} \widetilde{\mathbb{D}}, Q_{p, i}^{\prime} \in Q_{p, i}^{\prime}(0)+$ $\sum_{j} \Omega_{j} \widetilde{\mathbb{D}}, Q_{p, i}(0), Q_{p, i}^{\prime}(0) \in \mathbb{C}$, we obtain by applying rad:

$$
\operatorname{rad}(P)=\sum_{k \geq 0}\left(\sum_{i \geq 0} Q_{k, i}(0) \theta^{i}\right) z^{k}+\sum_{p>0}\left(\sum_{i \geq 0} Q_{p, i}^{\prime}(0) \theta^{i}\right) \delta^{p}
$$

Recall from $\S 2.2$ that there exists a filtration on $R$ such that $\operatorname{gr}(R)$ is isomorphic to $\mathbb{C}[X, Y, S] /\left(X Y-S^{n}\right)$ where $\operatorname{gr}(z) \equiv X, \operatorname{gr}(\delta) \equiv Y, \operatorname{gr}(\theta) \equiv S$ (up to some scalar). This implies easily that $R=\left(\bigoplus_{k>0} \mathbb{C}[\theta] z^{k}\right) \oplus\left(\bigoplus_{p>0} \mathbb{C}[\theta] \delta^{p}\right)$. Now suppose that $P \in J$, then $\operatorname{rad}(P)=0=\sum_{k>0}\left(\sum_{i>0} Q_{k, i}(0) \theta^{i}\right) z^{k}+\sum_{p>0}\left(\sum_{i>0} Q_{p, i}^{\prime}(0) \theta^{i}\right) \delta^{p}$ forces $\sum_{i \geq 0} Q_{k, i}(0) \theta^{i}=\sum_{i \geq 0} Q_{p, i}^{\prime}(0) \theta^{i}=0$, hence $Q_{k, i}(0)=Q_{p, i}^{\prime}(0)=0$ for all $k, p, i$. This shows that $Q_{k}, Q_{p}^{\prime} \in \sum_{i=0}^{r} \Omega_{0} \widetilde{\mathbb{D}}+\cdots+\Omega_{r} \widetilde{\mathbb{D}}$ and therefore $P \in$ $\sum_{i=0}^{r} \Omega_{i} \mathbb{D}$. Writing $P_{k} \in f^{k} \widetilde{\mathbb{D}}$ or $\Delta^{-k} \widetilde{\mathbb{D}}$ yields $P \in \sum_{i=0}^{r} \widetilde{\mathbb{D}} \Omega_{0}+\cdots+\widetilde{\mathbb{D}} \Omega_{r}$.

Remark 4.12. (1) In the case where $(\tilde{G}: V)$ is irreducible we have noticed that $\Omega_{r}=0$, see (4.6), thus

$$
J=\sum_{i=0}^{r-1} \mathbb{D} \Omega_{i}=\sum_{i=0}^{r-1} \Omega_{i} \mathbb{D} .
$$

(2) From $\left[\bar{\Theta}, f^{k}\right]=k f^{k}$ and $\left[\bar{\Theta}, \Delta^{k}\right]=-k \Delta^{k}$ we can deduce that

$$
\mathbb{D}[k]=\{D \in \mathbb{D}:[\bar{\Theta}, D]=k D\} .
$$

4.3. A Howe duality. The Howe duality for the Weil representation gives a bijection between irreducible finite dimensional representations of $\mathrm{SO}(n)$ and irreducible lowest weight $U(\mathfrak{s l}(2))$-modules. Algebraically, this result corresponds to the case $\left(\tilde{G}=\operatorname{SO}(n) \times \mathbb{C}^{*}: V=\mathbb{C}^{n}\right)$ : here the Lie subalgebra of $\mathcal{D}(V)$ generated by $f$ (quadratic form) and $\Delta$ (Laplacian) is isomorphic to $\mathfrak{s l}(2)$. More precisely we have the following result. Let $\mathcal{A} \cong \mathfrak{s l}(2)$ be this Lie algebra, denote by $H_{d} \subset H\left(V^{*}\right)$ the space of harmonic polynomials of degree $d$, i.e.:

$$
H_{d}=\left\{q \in S^{d}\left(V^{*}\right): \Delta(q)=0\right\} .
$$

Each $H_{d}$ is an irreducible $\mathrm{SO}(n)$-module and the $\mathcal{A} \times \mathrm{SO}(n)$-module $\mathbb{C}[V]$ decomposes as

$$
\mathbb{C}[V]=\bigoplus_{d} X(d+n / 2) \otimes H_{d}
$$

where $X(d+n / 2)$ is the irreducible lowest $\mathfrak{s l}(2)$-module of lowest weight $d+n / 2$.

This kind of duality has been extended by H. Rubenthaler [49] to a class of PHV, the so-called commutative parabolic PHV. They are associated to short gradings $\mathfrak{s}=\mathfrak{s}_{-1} \oplus \mathfrak{s}_{0} \oplus \mathfrak{s}_{1}$ on simple Lie algebras. The commutative parabolic PHV are 
irreducible, $\mathrm{MF}$ and satisfy $\operatorname{dim} V / / G=1$, thus Hypothesis B holds. We want to generalize the Howe duality to the more general class of representations $(\tilde{G}: V)$ satisfying only Hypothesis B. We therefore fix a representation $(\tilde{G}: V)$ satisfying this hypothesis, see $\$ 4.2$. We have indicated in the last column of the table of Appendix A the irreducible MF representations $(\tilde{G}: V)$ which are of commutative parabolic type.

Let

$$
\mathcal{A}=\operatorname{Lie}\langle f, \Delta\rangle \subset(\mathcal{D}(V),[,])
$$

be the Lie subalgebra generated by $f, \Delta$. Notice that $\mathcal{A} \subset \mathbb{D}$. Let $\tilde{\gamma}=\sum_{j=0}^{r} a_{j} \tilde{\lambda}_{j}$. Recall that $V(\tilde{\gamma})=U(\mathfrak{g}) \cdot h^{\tilde{\gamma}}$; we then set:

$$
\mathrm{a}=\left(a_{0}, \ldots, a_{r}\right), \quad h^{\tilde{\gamma}}=h^{\mathrm{a}}=f^{a_{0}} h_{1}^{a_{1}} \ldots h_{r}^{a_{r}}, \quad V(\tilde{\gamma})=V(\mathrm{a})=V\left(a_{0}, \ldots, a_{r}\right) .
$$

Let $b \in \mathbb{N}$, by Lemma $4.4(\mathrm{~b})$, the operator $\Delta^{b}$ acts as follows: $\Delta^{b}(V(\mathrm{a}))=0$ if $a_{0}<b, \Delta^{b}: V\left(a_{0}, \ldots, a_{r}\right) \stackrel{\sim}{\longrightarrow} V\left(a_{0}-b, a_{1}, \ldots, a_{r}\right)$ if $b \leq a_{0}$, and in the latter case $\Delta^{b}\left(h^{a}\right) \in \mathbb{C}^{*} f^{a_{0}-b} h_{1}^{a_{1}} \cdots h_{r}^{a_{r}}$ is a highest weight vector in $V\left(a_{0}-b, \ldots, a_{r}\right)$. Obviously, the multiplication by $f^{b}$ gives an isomorphism $f^{b}: V\left(a_{0}, \ldots, a_{r}\right) \stackrel{\sim}{\longrightarrow} V\left(a_{0}+\right.$ $\left.b, a_{1}, \ldots, a_{r}\right)$ of $\tilde{G}$-modules. Define:

$$
\mathcal{A}_{j}=\left\{D \in \mathcal{A}: D\left(V\left(a_{0}, \ldots, a_{r}\right)\right) \subset V\left(a_{0}+j, a_{1}, \ldots, a_{r}\right) \text { for all } \mathrm{a} \in \mathbb{N}^{r+1}\right\} .
$$

It is clear that $\bigoplus_{j \in \mathbb{Z}} \mathcal{A}_{j} \subset \mathcal{A}$ and, by the previous remarks, $f^{b} \in \mathcal{A}_{b}, \Delta^{b} \in \mathcal{A}_{-b}$.

Remarks. 1) It is difficult to compute in the Lie algebra $\mathcal{A}$ because $[\Delta, f]=\psi(-\bar{\Theta})+$ $Q$ for some $Q \in \operatorname{Ker}(\mathrm{rad})$ which is not easy to calculate (recall that here $\psi(s)=$ $b(-s)-b(-s-1)$, cf. $(2.6))$. For example when $(\tilde{G}: V)=\left(\operatorname{GL}(n) \times \operatorname{SL}(n): \mathrm{M}_{n}(\mathbb{C})\right)$ P. Nang [41] has shown that (up to some scalar): $Q=\operatorname{trace}\left(\mathbf{x}^{\#} \boldsymbol{\partial}^{\#}\right)$ where $\mathbf{x}^{\#}$, resp. $\boldsymbol{\partial}^{\#}$, is the adjoint matrix of $\mathbf{x}=\left[x_{i j}\right]_{i j}$, resp. $\boldsymbol{\partial}=\left[\partial_{x_{i j}}\right]_{i j}$. (See also $[43$, Proposition 7] for the case $\left(\mathrm{GL}(2 m): \bigwedge^{2} \mathbb{C}^{2 m}\right)$.)

2) When $n=\operatorname{deg} f=2$ one has $\mathcal{A} \cong \mathfrak{s l}(2)$, thus $\operatorname{dim}_{\mathbb{C}} \mathcal{A}=3$.

The assertion (2) of the next proposition should be compared with [49, Théorème 3.1].

Proposition 4.13. (1) One has $\mathcal{A}_{k}=\mathcal{A} \cap \mathbb{D}[k], \mathcal{A}=\bigoplus_{k \in \mathbb{Z}} \mathcal{A}_{k}$. The Lie subalgebra $\mathcal{A}_{0}$ is abelian.

(2) Suppose $n \geq 3$, then $\operatorname{dim}_{\mathbb{C}} \mathcal{A}_{k}=\infty$ for all $k \in \mathbb{Z}$.

Proof. (1) Since $f, \Delta \in \mathcal{A}$ the relations $[\bar{\Theta}, f]=f$ and $[\bar{\Theta}, \Delta]=-\Delta$ show that $\operatorname{ad}(\bar{\Theta}): \mathbb{D} \rightarrow \mathbb{D}$ induces an endomorphism of $\mathcal{A}$. From $\mathcal{A} \subset \mathbb{D}$ we deduce that $\mathcal{A}=\bigoplus_{k \in \mathbb{Z}} \mathcal{A} \cap \mathbb{D}[k]$. let $P \in \mathbb{D}[k], P=f^{k} D$ or $\Delta^{-k} D$ be an element of $\mathcal{A} \cap \mathbb{D}[k]$. Then, using Remark $4.9(3)$, we see that $P \in \mathcal{A}_{k}$. The desired equalities follow easily. Since $\mathcal{A}_{0} \subset \widetilde{\mathbb{D}}$ and $\widetilde{\mathbb{D}}$ is a commutative algebra, cf. Theorem $4.7, \mathcal{A}_{0}$ is abelian.

(2) We claim that $\operatorname{rad}\left(\mathcal{A}_{j}\right)=\mathcal{L}_{j}$, where $\mathcal{L}_{j}$ is defined as in $\S 2.3$, i.e.: $\mathcal{L}=$ $\operatorname{Lie}\langle f, \Delta\rangle, \mathcal{L}_{i}=\left\{u \in \mathcal{L}: u\left(z^{m}\right) \in \mathbb{C} z^{m+j}\right.$ for all $\left.m \in \mathbb{N}\right\}$ (with the convention that $\mathbb{C} z^{m+j}=(0)$ when $\left.m+j<0\right)$. Note first that $\operatorname{rad}(\mathcal{A})=\operatorname{Lie}\langle\operatorname{rad}(f), \operatorname{rad}(\Delta)\rangle=\mathcal{L}$. Let $D \in \mathcal{A}_{j}$, then $\operatorname{rad}(D)\left(z^{m}\right)=\psi\left(D\left(f^{m}\right)\right)$. Observe that $f^{m} \in V(m, 0, \ldots, 0)$, hence $D\left(f^{m}\right) \in V(m+j, 0, \ldots, 0)$, which is $(0)$ is $m+j<0$ and $\mathbb{C} f^{m+j}$ if $m+j \geq 0$. Thus $\operatorname{rad}(D) \in \mathbb{C} z^{m+j}$ and $\operatorname{rad}\left(\mathcal{A}_{j}\right) \subset \mathcal{L}_{j}$. It follows that $\operatorname{rad}(\mathcal{A})=\sum_{j} \operatorname{rad}\left(\mathcal{A}_{j}\right) \subset$ $\oplus_{j} \mathcal{L}_{j} \subset \mathcal{L}=\operatorname{rad}(\mathcal{A})$. Therefore $\operatorname{rad}\left(\mathcal{A}_{j}\right)=\mathcal{L}_{j}$ for all $j$ (and $\left.\mathcal{L}=\bigoplus_{j} \mathcal{L}_{j}\right)$. Now, Proposition 2.5 yields the desired assertion.

Set $\mathcal{A}_{+}=\bigoplus_{k>0} \mathcal{A}_{k}, \mathcal{A}_{-}=\bigoplus_{k<0} \mathcal{A}_{k}$. We then have a triangular decomposition $\mathcal{A}=\mathcal{A}_{-} \oplus \mathcal{A}_{0} \oplus \mathcal{A}_{+}$which enables us to introduce the notion of a lowest weight $\mathcal{A}$-module, see [49]. As usual in this situation the weights will be elements of the linear dual space $\mathcal{A}_{0}^{*}$ of the abelian Lie algebra $\mathcal{A}_{0}$. 
Definition 4.14. The $\mathcal{A}$-module $X$ is a lowest weight module if there exist $x \in X$ and $\varphi \in \mathcal{A}_{0}^{*}$ such that: $X=U(\mathcal{A}) . x, \mathcal{A}_{-} . x=0, a . x=\varphi(a) x$ for all $a \in \mathcal{A}_{0}$.

The next theorem generalizes [49, Proposition 4.2]; it gives a Howe duality for MF representations with a one dimensional quotient (see also [10, Corollary 4.5.17]). Recall that $H\left(V^{*}\right)=\bigoplus_{\gamma \in \Gamma} V(\gamma)$, where $V(\gamma) \cong E(p(\gamma))$, is equal to the space of harmonic elements, i.e. $\left\{\varphi \in \mathbb{C}[V]: \Delta_{0}(\varphi)=\cdots=\Delta_{m}(\varphi)=0\right\}$, cf. (4.2) and Proposition 4.5.

Theorem 4.15. Let $(\tilde{G}: V)$ satisfying Hypothesis $B$. The $\mathcal{A} \times \mathfrak{g}$-module $\mathbb{C}[V]$ decomposes as $\mathbb{C}[V] \cong \bigoplus_{\gamma \in \Gamma} X(\gamma) \otimes E(p(\gamma))$, where $X(\gamma)=U(\mathcal{A}) \cdot h^{\gamma}$ is an irreducible lowest weight $\mathcal{A}$-module. Moreover: $X(\gamma) \cong X\left(\gamma^{\prime}\right) \Longleftrightarrow \gamma=\gamma^{\prime}$.

Proof. Recall that $\tilde{\Gamma}=\mathbb{N} \tilde{\lambda}_{0} \oplus \Gamma, \Gamma=\bigoplus_{i=1}^{r} \mathbb{N} \tilde{\lambda}_{i}$. Let $\gamma=\sum_{i=1}^{r} a_{i} \tilde{\lambda}_{i} \in \Gamma$; set $P_{\gamma}=$ $\gamma+\mathbb{N} \tilde{\lambda}_{0}$. Then $h^{\gamma}=h_{1}^{a_{1}} \cdots h_{r}^{a_{r}}$ and $V(\gamma)=U(\mathfrak{g}) \cdot h^{\gamma} \cong E(p(\gamma))$, see Lemma 4.2. From $f^{b} h^{\gamma}=h^{\left(b, a_{1}, \ldots, a_{r}\right)}$ and $\Delta^{b}\left(f^{a_{0}} h_{1}^{a_{1}} \cdots h_{r}^{a_{r}}\right) \in \mathbb{C}^{*} f^{a_{0}-b} h_{1}^{a_{1}} \cdots h_{r}^{a_{r}}$ when $a_{0} \geq b$, and 0 when $a_{0}<b$, we get that $X(\gamma)=U(\mathcal{A}) \cdot h^{\gamma}=\bigoplus_{\mu \in P_{\gamma}} \mathbb{C} h^{\mu}$ is an irreducible $U(\mathcal{A})$-module. Let $D \in \mathcal{A}_{-j}=\mathcal{A} \cap \mathbb{D}[-j], j>0$, and write $D=D^{\prime} \Delta^{j}, D^{\prime} \in \widetilde{\mathbb{D}}$. Then $D\left(h^{\gamma}\right)=D^{\prime} \Delta^{j}\left(h^{\gamma}\right)$ and we have noticed that $\Delta^{j}\left(h^{\gamma}\right)=0$, thus $\mathcal{A}_{-} \cdot h^{\gamma}=0$. When $D \in \mathcal{A}_{0}=\mathcal{A} \cap \widetilde{\mathbb{D}}$, Remark 4.9(3) gives that $D\left(h^{\gamma}\right)=\varphi(D) h^{\gamma} \in \mathbb{C} h^{\gamma}$. Since it is obvious that $\varphi \in \mathcal{A}_{0}^{*}, X(\gamma)$ is an irreducible lowest weight module.

By $[10$, Theorem 4.5.16] we know that the $\mathbb{D} \times \mathfrak{g}$-module $\mathbb{C}[V]$ has the following decomposition:

$$
\mathbb{C}[V]=\bigoplus_{\lambda \in \Lambda^{+}} \operatorname{Hom}_{\mathfrak{g}}(E(\lambda), \mathbb{C}[V]) \otimes_{\mathbb{C}} E(\lambda)
$$

where $\operatorname{Hom}_{\mathfrak{g}}(E(\lambda), \mathbb{C}[V])$ is either $(0)$ or a simple $\mathbb{D}$-module (the action being given by $D(\phi)(x)=D(\phi(x))$ for all $\left.\phi \in \operatorname{Hom}_{\mathfrak{g}}(E(\lambda), \mathbb{C}[V]), x \in E(\lambda)\right)$. Since the $G$ module $V(\tilde{\gamma}), \tilde{\gamma} \in \tilde{\Gamma}$, is isomorphic to $E(p(\tilde{\gamma}))$, we obtain that $\operatorname{Hom}_{\mathfrak{g}}(E(\lambda), \mathbb{C}[V])=$ (0) if $\lambda \notin p(\Gamma)=p(\tilde{\Gamma})$. If $\lambda \in p(\Gamma)$ the non zero elements of this $\mathbb{D}$-module identify with the $\mathfrak{g}$-highest weight vectors of weight $\lambda$ in $\mathbb{C}[V]$ through the map $\phi \mapsto \phi\left(v_{\lambda}\right)$, where $v_{\lambda}$ is a highest weight vector in $E(\lambda)$. It is easily seen that the $\mathfrak{g}$-highest weight vectors of weight $\lambda$ in $\mathbb{C}[V]$ are the $h^{\tilde{\gamma}}$ with $\tilde{\gamma}=k \tilde{\lambda}_{0}+\gamma^{\prime}, p\left(\gamma^{\prime}\right)=\lambda$. Recall that for $\gamma, \gamma^{\prime} \in \Gamma, p(\gamma)=p\left(\gamma^{\prime}\right) \Longleftrightarrow \gamma=\gamma^{\prime}$; therefore these $\mathfrak{g}$-highest weight vectors are the $h^{\tilde{\gamma}}$ with $\tilde{\gamma} \in P_{\gamma}=\gamma+\mathbb{N} \tilde{\lambda}_{0}$, where $\gamma \in \Gamma$ is such that $p(\gamma)=\lambda$. From the previous paragraph we then obtained that $\operatorname{Hom}_{\mathfrak{g}}(E(\lambda), \mathbb{C}[V]) \cong X(\gamma)$ as $\mathcal{A}$-module. The last assertion follows from [10, Theorem 4.5.12].

\section{D-MOdules On SOME PHV}

In this section we continue with a representation $(\tilde{G}: V)$ of the connected reductive group $\tilde{G}$ as in $\$ 3.1$.

\subsection{Representations of Capelli type. Let}

$$
\tau: \tilde{\mathfrak{g}}=\operatorname{Lie}(\tilde{G}) \longrightarrow \mathcal{D}(V)
$$

be the differential of the $\tilde{G}$-action. The elements $\tau(\xi)$ are linear derivations on $\mathbb{C}[V]$ given by:

$$
\tau(\xi)(\varphi)(v)=\frac{d}{d t}_{\mid t=0}\left(\mathrm{e}^{t \xi} \cdot \varphi\right)(v)=\frac{d}{d t}_{\mid t=0} \varphi\left(\mathrm{e}^{-t \xi} \cdot v\right),
$$

for all $\varphi \in \mathbb{C}[V], v \in V$. They are homogeneous of degree zero in the sense that $[\Theta, \tau(\xi)]=0$. The map $\tau$ yields a homomorphism $\tau: U(\tilde{\mathfrak{g}}) \rightarrow \mathcal{D}(V)$.

Recall that the group $\tilde{G}$ acts naturally on $\mathcal{D}(V)$; the differential of this action is given by $D \mapsto[\tau(\xi), D], \xi \in \tilde{\mathfrak{g}}, D \in \mathcal{D}(V)$. Therefore, a subspace $I \subset \mathcal{D}(V)$ is stable under $\tilde{G}$, resp. $G$, if and only if $[\tau(\tilde{\mathfrak{g}}, I] \subset I$, resp. $[\tau(\mathfrak{g}, I] \subset I$. It is then 
clear that $\widetilde{\mathbb{D}}=\mathcal{D}(V)^{\tilde{G}}=\{D \in \mathcal{D}(V):[\tau(\tilde{\mathfrak{g}}), D]=0\} \subset \mathbb{D}=\mathcal{D}(V)^{G}=\{D \in$ $\mathcal{D}(V):[\tau(\mathfrak{g}), D]=0\}$. In particular, if $Z(\tilde{\mathfrak{g}})=U(\tilde{\mathfrak{g}})^{\tilde{G}}$ is the centre of $U(\tilde{\mathfrak{g}})$, then $\tau(Z(\tilde{\mathfrak{g}})) \subset \widetilde{\mathbb{D}}$.

Following $[18,(10.3)]$ we make the following definition:

Definition 5.1. We say that $(\tilde{G}: V)$ is of Capelli type if:

- $(\tilde{G}: V)$ is irreducible and multiplicity free;

- $\tau(Z(\tilde{\mathfrak{g}}))=\widetilde{\mathbb{D}}$.

Remarks 5.2. (1) By Howe and Umeda [18], in the list of $(\tilde{G}: V)$ which are irreducible and MF we have: three among the thirteen cases are not of Capelli type; two among the ten cases such that $\operatorname{dim} V / / G=1$ are not of Capelli type. (Thus we are interested in eight cases of the table in Appendix A.)

(2) This definition originates in the case $\left(\tilde{G}=\mathrm{GL}(n) \times \mathrm{SL}(n): V=\mathrm{M}_{n}(\mathbb{C})\right)$ where the writing of $E_{0}=f \Delta=\operatorname{det}\left(x_{i j}\right) \operatorname{det}\left(\partial_{i j}\right)$ as an element of $\tau(Z(\tilde{\mathfrak{g}}))$ is the "classical" Capelli identity.

Recall the morphism $\operatorname{rad}: \mathcal{D}(V)^{G} \rightarrow \mathcal{D}(V / / G)$. By definition $\tau(\mathfrak{g})\left(\mathbb{C}[V]^{G}\right)=0$, thus one always has:

$$
[\mathcal{D}(V) \tau(\mathfrak{g})]^{G} \subset J=\operatorname{Ker}(\mathrm{rad}) .
$$

When $(\tilde{G}: V)$ satisfies Hypothesis B, we have computed in Theorem 4.11 the ideal $J \subset \mathbb{D}:$

$$
J=\sum_{i=0}^{r} \mathbb{D} \Omega_{i}=\sum_{i=0}^{r} \Omega_{i} \mathbb{D}
$$

where the $\Omega_{i}$ 's are defined in (4.5). When $(\tilde{G}: V)$ is irreducible we observed in Remark 4.12(1) that we can number these operators so that $\Omega_{r}=0$, hence $J=$ $\sum_{i=0}^{r-1} \mathbb{D} \Omega_{i}$; the next proposition gives a more useful description if, moreover, $(\tilde{G}: V)$ is of Capelli type, i.e. one of the eight cases mentioned in Remark 5.2(1).

Proposition 5.3. Let $(\tilde{G}: V)$ be of Capelli type and such that $\operatorname{dim} V / / G=1$. Then:

$$
\operatorname{Ker}(\mathrm{rad})=[\mathcal{D}(V) \tau(\mathfrak{g})]^{G} .
$$

Proof. In the irreducible case the centre $C$ of $\tilde{G}$ acts by scalars on $V$ and we may assume that: $\tilde{G}=G C$ with $C=\mathbb{C}^{*}$. Write $\tilde{\mathfrak{g}}=\mathfrak{g} \oplus \mathfrak{c}, \mathfrak{c}=\operatorname{Lie}(C)=\mathbb{C} \zeta$. Since $\mathbb{C}[V]^{G}=\mathbb{C}[f]$ and $f$ is not $\tilde{G}$-invariant one can also suppose that $\tau(\zeta)=\bar{\Theta}=\frac{1}{n} \Theta$.

Write $Z(\tilde{\mathfrak{g}})=Z(\mathfrak{g})[\zeta]$ and $Z(\mathfrak{g})=\mathbb{C} \oplus Z_{+}(\mathfrak{g})$ where $Z_{+}(\mathfrak{g})=[U(\mathfrak{g}) \mathfrak{g}]^{G}$. The previous paragraph implies that $\tau(Z(\tilde{\mathfrak{g}}))=\mathbb{C}[\bar{\Theta}]+\tau\left(Z_{+}(\mathfrak{g})\right)[\bar{\Theta}]$ with $\tau\left(Z_{+}(\mathfrak{g})\right)=$ $[U(\tau(\mathfrak{g})) \tau(\mathfrak{g})]^{G} \subset[\mathcal{D}(V) \tau(\mathfrak{g})]^{G}$. As recalled above we already know that $J=$ $\sum_{i=0}^{r-1} \mathbb{D} \Omega_{i}, \Omega_{i} \in \widetilde{\mathbb{D}}, 0 \leq i \leq r-1$. By hypothesis $\tau(Z(\tilde{\mathfrak{g}}))=\widetilde{\mathbb{D}}$, thus we can write each $\Omega_{j}$ as follows:

$$
\Omega_{j}=\sum_{k \geq 0} \bar{\Theta}^{k} P_{j, k}, \quad P_{j, k}=p_{j, k}+P_{j, k}^{\prime}, p_{j, k} \in \mathbb{C}, P_{j, k}^{\prime} \in \tau\left(Z_{+}(\mathfrak{g})\right) .
$$

Recall that $[\mathcal{D}(V) \tau(\mathfrak{g})]^{G} \subset J$; thus we have $\operatorname{rad}\left(P_{j, k}^{\prime}\right)=0$ and we obtain: $\operatorname{rad}\left(\Omega_{j}\right)=$ $\sum_{k \geq 0} \theta^{k} p_{j, k}=0$ in $R=\mathbb{C}[z, \delta, \theta]$. Therefore $p_{j, k}=0$ for all $k \geq 0$, which gives $\Omega_{j}=\sum_{k \geq 0} \bar{\Theta}^{k} P_{j, k}^{\prime} \in[\mathcal{D}(V) \tau(\mathfrak{g})]^{G}$ and $J \subset[\mathcal{D}(V) \tau(\mathfrak{g})]^{G}$.

5.2. Application to $D$-modules. In this subsection we assume that $(\tilde{G}: V)$ satisfies Hypothesis $B$, hence $(\tilde{G}: V)$ is $M F, \mathbb{C}[V]^{G}=\mathbb{C}[f], f \notin \mathbb{C}[V]^{\tilde{G}}$.

Recall from Theorem 4.11 that $\mathbb{D}=\bigoplus_{k \in Z} \mathbb{D}[k]$. We have seen (Lemma 4.10) that if $D \in \widetilde{\mathbb{D}}$ there exists a polynomial $b_{D}(s)$ such that $D=b_{D}(\bar{\Theta})+D_{1}, D_{1} \in$ $J=\operatorname{Ker}(\mathrm{rad})$. 
Fix $P \in \mathbb{D}[k]$ and write $P=D Q_{k}, Q_{k}=f^{k}$ if $k \geq 0, Q_{k}=\Delta^{-k}$ if $k<0, D \in \widetilde{\mathbb{D}}$. Then:

$$
P=b_{D}(\bar{\Theta}) Q_{k}+P_{1}, \quad P_{1}=D_{1} Q_{k} \in J .
$$

Observe that, since $\Delta\left(f^{m}\right)=b^{*}(m) f^{m-1}$,

$$
P\left(f^{m}\right)= \begin{cases}b_{D}(m+k) f^{m+k} & \text { if } k \geq 0 ; \\ b^{*}(m) b^{*}(m-1) \cdots b^{*}(m+k+1) b_{D}(m+k) f^{m+k} & \text { if } k<0 .\end{cases}
$$

Therefore if we set $a_{P}(s)=b_{D}(s+k)$ if $k \geq 0$, or $a_{P}(s)=b_{D}(s+k) \prod_{j=0}^{k+1} b^{*}(s+j)$ if $k<0$, then $\operatorname{deg} a_{P}=\operatorname{deg} b_{P}$ or $\operatorname{deg} b_{P}+n k$, and $P\left(f^{m}\right)=a_{P}(m) f^{m+k}$. Notice that $a_{Q_{k}}(s)=1$ if $k \geq 0, a_{Q_{k}}(s)=\prod_{j=0}^{k+1} b^{*}(s+j)$ if $k<0$, thus $a_{P}(s)=b_{D}(s+k) a_{Q_{k}}(s)$.

When $(\tilde{G}: V)=\left(\mathrm{GL}(n): S^{2} \mathbb{C}^{n}\right)$, similar results were obtained by Masakazu Muro in [36, Proposition 3.8], where our polynomial $a_{P}(s)$ is denoted by $b_{P}(s)$.

Definition 5.4. Let $P \in \mathbb{D}[k]$ be as above and define the $D$-module associated to $P$ by:

$$
\mathcal{M}_{P}=\mathcal{D}(V) /(\mathcal{D}(V) \tau(\mathfrak{g})+\mathcal{D}(V) P)=\mathcal{D}(V) / I_{P},
$$

where $I_{P}=\mathcal{D}(V) \tau(\mathfrak{g})+\mathcal{D}(V) P$.

Remark. Let $I \subset \mathcal{D}(V)$ be a left ideal containing $\mathcal{D}(V) \tau(\mathfrak{g})$. Since the condition $[\tau(\mathfrak{g}), I] \subset I$ is satisfied, the group $G$ acts naturally on $I$ and therefore on $M=\mathcal{D}(V) / I$. Furthermore, the differential of this action is given by the left multiplication on $M$ by $\tau(\xi), \xi \in \mathfrak{g}$. It follows, see [17, §II.2, Theorem], that $M$ is a $G$-equivariant $D$-module on $V$ (cf. [17] for the definition). This is in particular true for $\mathcal{M}_{P}$.

Following $[33,34,35,36]$ we want to study the solutions of the differential system associated to $\mathcal{M}_{P}$. We first need to study the characteristic variety on $\mathcal{M}_{P}$.

Recall that $\mathcal{D}(V)$ is filtered by the order of differential operators, see [4, 16], and that the associated graded ring of $\mathcal{D}(V)$ identifies with $\mathbb{C}\left[T^{*} V\right]$, where $T^{*} V=$ $V \times V^{*}$ is the cotangent bundle of $V$. If $u \in \mathcal{D}(V)$ we denote its order by ord $u$ and its principal symbol by $\sigma(u) \in \mathbb{C}\left[T^{*} V\right]=S\left(V^{*}\right) \otimes S(V)$. Let $M$ be a finitely generated $\mathcal{D}(V)$-module, then one can endow $M$ with a good filtration and one defines the characteristic variety of $M$, denoted by $\mathrm{Ch} M$, as being the support in $T^{*} M$ of the associated graded module (see, e.g., [16, §I.3]). When $M=\mathcal{D}(V) / I, \operatorname{Ch} M \subset T^{*} M$ is the variety of zeroes of the symbols $\sigma(u), u \in I$. Recall that if $\operatorname{dim} \operatorname{Ch} M \leq$ $\operatorname{dim} V$ the $D$-module $M$ is called holonomic (one always have $\operatorname{dim} \operatorname{Ch} M \geq \operatorname{dim} \bar{V}$ if $M \neq(0))$.

Remark. Let $v \in V, v^{*} \in V^{*}, \xi \in \tilde{\mathfrak{g}}$. One has:

$$
\sigma(\Theta)\left(v, v^{*}\right)=\left\langle v^{*}, v\right\rangle, \quad \sigma(\tau(\xi))\left(v, v^{*}\right)=-\left\langle\xi . v^{*}, v\right\rangle=\left\langle v^{*}, \xi . v\right\rangle .
$$

Lemma 5.5. Let $k \in \mathbb{Z}$ and $P=D Q_{k}$ be as above.

(a) There exists $Q_{k}^{\prime} \in J$ and $q_{k}(s) \in \mathbb{C}[s]$ such that $Q_{k} Q_{-k}=q_{k}(\bar{\Theta})+Q_{k}^{\prime}$, $\operatorname{ord}\left(Q_{k} Q_{-k}\right)=\operatorname{ord}\left(Q_{-k} Q_{k}\right)=\operatorname{deg} q_{k}=|k| n$.

(b) Write $P=b_{D}(\bar{\Theta}) Q_{k}+P_{1}$ as in (5.1) and set $P_{0}=b_{D}(\bar{\Theta}) Q_{k}$. Then $P_{0} Q_{-k}=$ $b_{D}(\bar{\Theta}) q_{k}(\bar{\Theta})+P_{2}$ with $P_{2} \in J$ and ord $P_{2} \leq \operatorname{ord}\left(P_{0} Q_{-k}\right)=\operatorname{deg}\left(b_{D} q_{k}\right)$.

Proof. (a) Let $m \in \mathbb{N}$. Then:

$$
Q_{k} Q_{-k}\left(f^{m}\right)=a_{Q_{-k}}(m) Q_{k}\left(f^{m-k}\right)=a_{Q_{-k}}(m) a_{Q_{k}}(m-k) f^{m} .
$$

Set $q_{k}(s)=a_{Q_{-k}}(s) a_{Q_{k}}(s-k) \in \mathbb{C}[s]$. The previous computation yields $Q_{k} Q_{-k}=$ $q_{k}(\bar{\Theta})+Q_{k}^{\prime}$ with $Q_{k}^{\prime} \in J$. Since $\operatorname{deg} a_{Q_{k}}=1$ or $-n k$ (if $k \geq 0$ or $<0$ ) we get that $\operatorname{deg} q_{k}=|k| n$. On the other hand, $Q_{k} Q_{-k}=f^{k} \Delta^{-k}$ or $\Delta^{k} f^{-k}$ has order ord $\Delta^{|k|}$, i.e. $|k| n=\operatorname{deg} q_{k}$. This implies in particular that ord $Q_{k}^{\prime} \leq \operatorname{ord}\left(Q_{k} Q_{-k}\right)$. 
(b) From (a) we obtain $P_{0} Q_{-k}=b_{D}(\bar{\Theta}) q_{k}(\bar{\Theta})+P_{2}, P_{2}=b_{D}(\bar{\Theta}) Q_{k}^{\prime} \in J$. One has: $\operatorname{ord}\left(P_{0} Q_{-k}\right)=\operatorname{ord} P_{0}+\operatorname{ord} Q_{-k}=\operatorname{deg} b_{D}+\operatorname{ord} Q_{k}+\operatorname{ord} Q_{-k}=\operatorname{deg} b_{D}+$ $\operatorname{ord}\left(Q_{k} Q_{-k}\right)$. Therefore, ord $P_{2}=\operatorname{deg} b_{D}+\operatorname{ord} Q_{k}^{\prime} \leq \operatorname{deg} b_{D}+\operatorname{ord}\left(Q_{k} Q_{-k}\right)=$ $\operatorname{ord}\left(P_{0} Q_{-k}\right)$, as desired.

As in [45, Section 3] define the commuting varieties of $(\tilde{G}: V)$ and $(G: V)$ by: $\tilde{\mathcal{C}}(V)=\left\{\left(v, v^{*}\right) \in T^{*} V:\left\langle v^{*}, \tilde{\mathfrak{g}} \cdot v\right\rangle=0\right\}, \quad \mathcal{C}(V)=\left\{\left(v, v^{*}\right) \in T^{*} V:\left\langle v^{*}, \mathfrak{g} \cdot x\right\rangle=0\right\}$.

Recall that $(\tilde{G}: V)$ is MF; this implies [21] that $\tilde{G}$ has finitely many orbits in $V$, denoted by $O_{1}, \ldots, O_{t}$. Set $T_{O_{i}}^{*} V=\left\{\left(v, v^{*}\right) \in T^{*} V: v \in O_{i},\left\langle v^{*}, \tilde{\mathfrak{g}} \cdot v\right\rangle=0\right\}$. By [47], see also [45, Theorem 3.2], we have the following result:

Theorem 5.6. The irreducible components of $\tilde{\mathcal{C}}(V)$ are the closures of the conormal bundles of the orbits, i.e. the $\mathcal{C}_{i}=\overline{T_{O_{i}}^{*} V}$. In particular, $\tilde{\mathcal{C}}(V)$ is equidimensional of dimension $\operatorname{dim} V$.

Remark 5.7. Set $\mathcal{C}(V)^{\prime}=\left\{\left(v, v^{*}\right) \in T^{*} V: \sigma(u)\left(v, v^{*}\right)=0\right.$ for all $\left.u \in \mathcal{D}(V) \tau(\mathfrak{g})\right\}$. Thus $\mathcal{C}(V)^{\prime}$ is the characteristic variety of the $\mathcal{D}(V)$-module

$$
\mathcal{N}=\mathcal{D}(V) / \mathcal{D}(V) \tau(\mathfrak{g})
$$

Let $P \in \mathbb{D}[k]$. Then we clearly have:

$$
\tilde{\mathcal{C}}(V) \subset \mathcal{C}(V), \quad \operatorname{Ch} \mathcal{M}_{P} \subset \mathcal{C}(V)^{\prime} \subset \mathcal{C}(V) .
$$

We will now assume that $(\tilde{G}: V)$ is irreducible. This means that $(\tilde{G}: V)$ is one of the cases (1) to (10) in the table of Appendix A. We may assume here that $\tilde{G}=G C, C \cong \mathbb{C}^{*}$. We then write $\tilde{\mathfrak{g}}=\mathfrak{g} \oplus \mathbb{C} \zeta, \mathfrak{c}=\operatorname{Lie}(C)=\mathbb{C} \zeta$ where $\zeta$ is chosen such that $\tau(\zeta)=\bar{\Theta}$ (i.e. $\zeta$ acts as $\frac{1}{n} \operatorname{id}_{V}$ on $V$ ).

Corollary 5.8. Under the previous hypothesis:

$-\operatorname{dim} \mathcal{C}(V)=\operatorname{dim} V+1$

- the module $\mathcal{N}$ is not holonomic, i.e. $\operatorname{dim} \mathcal{C}(V)^{\prime}=\operatorname{dim} V+1$.

Proof. By [21, Theorem 1] the representation $(G: V)$ is visible, i.e. $\{v \in V: f(v)=$ $0\}$ contains a finite number of $G$-orbits. Then, since $\mathbb{C}(V)^{G}=\mathbb{C}(f)$ and $f$ is non constant, [44, Theorems $2.3 \& 3.1$, Corollary 2.5] yield $\operatorname{dim} \mathcal{C}(V)=\operatorname{dim} V+1$.

Recall that if $M$ is any $\mathcal{D}(V)$-module one can identify $\operatorname{Hom}_{\mathcal{D}(V)}(\mathcal{N}, M)$ with the space $\{x \in M: \tau(\mathfrak{g}) \cdot x=0\}$. In particular, $\operatorname{Hom}_{\mathcal{D}(V)}(\mathcal{N}, \mathbb{C}[V]) \equiv \mathbb{C}[V]^{G}=$ $\mathbb{C}[f]$. If $\mathcal{N}$ were holonomic we would have $\operatorname{dim}_{\mathbb{C}} \operatorname{Hom}_{\mathcal{D}(V)}(\mathcal{N}, \mathbb{C}[V])<\infty$, cf. [32, Theorem 9.5.5], which is absurd.

Assume that $(\tilde{G}: V)$ is of Capelli type and let $P=b_{D}(\bar{\Theta}) Q_{k}+P_{1}, P_{1} \in J$, as in (5.1). By Proposition 5.3, $I_{P}=\mathcal{D}(V) \tau(\mathfrak{g})+\mathcal{D}(V) b_{D}(\bar{\Theta}) Q_{k}$. Therefore:

$$
\mathcal{M}\left(b_{D}, k\right)=\mathcal{M}_{P}=\mathcal{D}(V) /\left(\mathcal{D}(V) \tau(\mathfrak{g})+\mathcal{D}(V) b_{D}(\bar{\Theta}) Q_{k}\right)
$$

depends only on the polynomial $b_{D}(s)$ and the integer $k$. We need to know in which case $\mathcal{M}\left(b_{D}, k\right)$ is holonomic.

Theorem 5.9. Assume that $(\tilde{G}: V)$ is of Capelli type and let $P=b_{D}(\bar{\Theta}) Q_{k}+P_{1}$ with $P_{1} \in \operatorname{Ker}(\mathrm{rad})$. The following are equivalent:

$$
\text { (i) } b_{D}(s) \neq 0 ; \text { (ii) } \mathcal{M}\left(b_{D}, k\right)=\mathcal{M}_{P} \text { is holonomic. }
$$

In this case $\mathrm{Ch} \mathcal{M}_{P} \subset \tilde{\mathcal{C}}(V)$ is a union of $\mathcal{C}_{i}$ 's.

Proof. Suppose that $b_{D}=0$, then $\mathcal{M}\left(b_{D}, k\right)=\mathcal{N}$ is not holonomic. Conversely, suppose $b_{D} \neq 0$. We are going to show that $\mathcal{M}_{P} \subset \tilde{\mathcal{C}}(V)$, then Theorem 5.6 will give the result. 
Set $\alpha=\sigma(\bar{\Theta})=\sigma(\tau(\zeta)) \in \mathbb{C}\left[T^{*} V\right]$, hence $\alpha\left(v, v^{*}\right)=\frac{1}{n}\left\langle v^{*}, v\right\rangle$. Since $\tilde{\mathfrak{g}}=\mathfrak{g} \oplus \mathbb{C} \zeta$, we have $\tilde{\mathcal{C}}(V)=\mathcal{C}(V) \cap \alpha^{-1}(0)$. Using the notation of Lemma 5.5 we set $P_{0}=$ $b_{D}(\bar{\Theta}) Q_{k}, P_{0} Q_{-k}=b_{D}(\bar{\Theta}) q_{k}(\bar{\Theta})+P_{2}$ with $P_{2} \in J$. Notice that $\mathcal{M}_{P}=\mathcal{M}_{P_{0}}$. Since $b_{D} \neq 0$, we can write $h(s)=b_{D}(s) q_{k}(s)=h_{d} s^{d}+h_{d-1} s^{d-1}+\cdots, d=\operatorname{deg} h(s)=$ $\operatorname{deg} b_{D}(s)+\operatorname{deg} q_{k}(s)=\operatorname{deg} b_{D}(s)+|k| n \geq 0$. Recall that ord $P_{2} \leq \operatorname{ord} P_{0} Q_{k}=d$, therefore $\sigma\left(P_{0} Q_{k}\right)=\sigma(h(\bar{\Theta}))$ or $\sigma(h(\bar{\Theta}))+\sigma\left(P_{2}\right)$ (depending on ord $P_{2}<d$ or ord $\left.P_{2}=d\right)$.

If $d=0$, we get $k=0, b_{D} \in \mathbb{C}^{*}$, thus $\mathcal{M}_{P}=(0)$ and the claim is obvious. Suppose $d \geq 1$ and let $\left(v, v^{*}\right) \in \operatorname{Ch} \mathcal{M}_{P} \subset \operatorname{Ch} \mathcal{N}=\mathcal{C}(V)^{\prime}$. From $P_{2} \in J=$ $[\mathcal{D}(V) \tau(\mathfrak{g})]^{G} \subset \mathcal{D}(V) \tau(\mathfrak{g})$ we know that $\sigma\left(P_{2}\right)\left(v, v^{*}\right)=0$. Therefore, $\sigma\left(P_{0}\right)\left(v, v^{*}\right)=$ 0 implies

$$
\begin{aligned}
0 & =\sigma\left(P_{0}\right)\left(v, v^{*}\right) \sigma\left(Q_{-k}\right)\left(v, v^{*}\right)=\sigma\left(P_{0} Q_{-k}\right)\left(v, v^{*}\right)=\sigma(h(\bar{\Theta}))\left(v, v^{*}\right) \\
& =h_{d} \sigma(\bar{\Theta})^{d}\left(v, v^{*}\right)=h_{d} \alpha\left(v, v^{*}\right)^{d} .
\end{aligned}
$$

Hence $\alpha\left(v, v^{*}\right)=0$ and this proves $\left(v, v^{*}\right) \in \mathcal{C}(V)^{\prime} \cap \alpha^{-1}(0) \subset \tilde{\mathcal{C}}(V)$, as desired.

Remark 5.10. The previous result generalizes the main step in the proof of [36, Theorem 4.1] (see also [36, Theorem 6.1 and Remark 6.1]). Indeed the homogeneous elements of degree $k n$ in [36] are the elements of $\mathbb{D}[k]$ and [36, Lemma 4.1] shows, when $(\tilde{G}: V)=\left(\mathrm{GL}(n): S^{2} \mathbb{C}^{n}\right)$, that $\mathcal{M}_{P}$ is holonomic when $P \neq 0$ and $\operatorname{deg} a_{P}=$ ord $P$. Since $a_{P}(s)=b_{D}(s+k) a_{Q_{k}}(s) \neq 0 \Longleftrightarrow b_{D}(s) \neq 0$, Theorem 5.9 ensures that a more general result holds for representations of Capelli type; notice that the example given [36, Remark 4.1] is $P=\Omega_{0}=f \Delta-b^{*}(\bar{\Theta}) \in J$, hence $\mathcal{M}_{P}=\mathcal{N}$ is not holonomic. Observe also that Theorem 5.9 is proved in [33, Proposition 2.1] in the case $\left(b_{D}=1, k \geq 0\right)$.

5.3. Solutions of invariant differential equations. We continue with a representation $(\tilde{G}: V)$ of Capelli type. Let $\left(\tilde{G}_{\mathbb{R}}: V_{\mathbb{R}}\right)$ be a real form of $(\tilde{G}: V)$ in the sense of $[25, \S 4.1$, Proposition 4.1], cf. also [33, §1.2] and [13, §4.2\& \&4.3]. Such a form always exists. Let $M$ be a finitely generated $\mathcal{D}(V)$-module of the form $\mathcal{D}(V) / I$. Denote by $\mathcal{B}_{V_{\mathbb{R}}}$ the space of hyperfunctions on $V_{\mathbb{R}}$ (see, e.g., [13, §4.1]). Then $\mathcal{B}_{V_{\mathbb{R}}}$ is a $\mathcal{D}(V)$-module and the "hyperfunction solutions space" of $M$ is:

$$
\operatorname{Sol}\left(M, \mathcal{B}_{V_{\mathbb{R}}}\right)=\left\{T \in \mathcal{B}_{V_{\mathbb{R}}}: D . T=0 \text { for all } D \in I\right\} \equiv \operatorname{Hom}_{\mathcal{D}_{V}}\left(M, \mathcal{B}_{V_{\mathbb{R}}}\right) .
$$

Notice that since any distribution on $V_{\mathbb{R}}$ can be viewed as a hyperfunction, the "distribution solutions space" of $M$ is contained in $\operatorname{Sol}\left(M, \mathcal{B}_{V_{\mathbb{R}}}\right)$. An element $T$ of $\mathcal{B}_{V_{\mathbb{R}}}$ such that $\tau(\mathfrak{g}) \cdot T=0$ is said to be $\mathfrak{g}$-invariant; it is called quasi-homogeneous if there exist $t \in \mathbb{N}, \mu \in \mathbb{C}$ such that $(\Theta-\mu)^{t} . T=0$. Assume that $I=\mathcal{D}(V) \tau(\mathfrak{g})+$ $\mathcal{D}(V) P$ for some $P \in \mathcal{D}(V)$, then $\operatorname{Sol}\left(M, \mathcal{B}_{V_{\mathbb{R}}}\right)$ identifies with the space of $\mathfrak{g}$-invariant hyperfunctions $T$ which are solutions of the equation P.T $=0$.

The next corollary has been proved by M. Muro [36, Theorem 4.1] for the real form $\left(\tilde{G}_{\mathbb{R}}=\operatorname{GL}(n, \mathbb{R}), V_{\mathbb{R}}=\operatorname{Sym}_{n}(\mathbb{R})\right)$ of $(\tilde{G}: V)=\left(\operatorname{GL}(n): S^{2} \mathbb{C}^{n}\right)$ when $P \neq 0$ and $\operatorname{deg} a_{P}=\operatorname{ord} P$. (See Remark 5.10 for more details.)

Corollary 5.11. Let $(\tilde{G}: V)$ be of Capelli type and let $\left(\tilde{G}_{\mathbb{R}}: V_{\mathbb{R}}\right)$ be a real form of $(\tilde{G}: V)$. Let $P \in \mathbb{D}[k]$ and write $P=b_{D}(\bar{\Theta}) Q_{k}+P_{1}, P_{1} \in \operatorname{Ker}(\mathrm{rad})$, as in (5.1). Assume that $b_{D} \neq 0$. Then, $\operatorname{Sol}\left(P, \mathcal{B}_{V_{\mathbb{R}}}\right)=\operatorname{Sol}\left(\mathcal{M}_{P}, \mathcal{B}_{V_{\mathbb{R}}}\right)=\left\{T \in \mathcal{B}_{V_{\mathbb{R}}}\right.$ : $T \mathfrak{g}$-invariant, P.T $=0\}$ is finite dimensional and has a basis of quasi-homogeneous elements; it depends only on the polynomial $b_{D}(s)$ and the integer $k$.

Proof. We merely repeat the proof of M. Muro (loc. cit.). A well-known result of M. Kashiwara (see [23, Théorème 5.1.6]) says that if $M$ holonomic $\operatorname{Sol}\left(M, \mathcal{B}_{V_{\mathbb{R}}}\right.$ ) is a finite dimensional $\mathbb{C}$-vector space. Therefore by combining the remarks above and Theorem 5.9 we obtain that $\mathcal{S}=\operatorname{Sol}\left(P, \mathcal{B}_{V_{\mathbb{R}}}\right)$ is finite dimensional. Now observe 
that $[\Theta, P]=k P$ and $[\Theta, \tau(\mathfrak{g})]=0$ imply that $\mathcal{S}$ is stable under the action of $\Theta$. Therefore $\mathcal{S}$ decomposes as a direct sum of spaces of the form $\operatorname{Ker}\left(\Theta-\mu \mathrm{id}_{\mathcal{S}}\right)^{t}$. We have noticed after (5.3) that $\mathcal{M}_{P}$ depends only on $b_{D}(s)$ and $k$, therefore it is also the case for $\mathcal{S}=\operatorname{Hom}_{\mathcal{D}_{V}}\left(\mathcal{M}_{P}, \mathcal{B}_{V_{\mathbb{R}}}\right)$.

5.4. Regular holonomic modules. Assume that $(\tilde{G}: V)$ is of Capelli type and $\operatorname{dim} V / / G=1$.

We filter $\mathcal{D}(V)$ by order of differential operators and we set $\mathcal{D}(V)_{j}=\{D \in$ $\mathcal{D}(V)$ : ord $D \leq j\}$. For sake of completeness we now recall some known (or easy) results.

Definition 5.12. Let $M$ be a finitely generated $\mathcal{D}(V)$-module.

$-M$ is monodromic if $\operatorname{dim}_{\mathbb{C}} \mathbb{C}[\Theta] . x<\infty$ for all $x \in M$.

- $M$ is homogeneous if there exists a $\Theta$-stable good filtration on $M$, i.e. a good filtration $F M=\left(F_{p} M\right)_{p \in \mathbb{N}}$ such that $\Theta . F_{p} M \subset F_{p} M$ for all $p$.

$-x \in M$ is quasi-homogeneous (of weight $\lambda$ ) if there exists $j \in \mathbb{N}$ and $\lambda \in \mathbb{C}$ such that $(\Theta-\lambda)^{j} \cdot x=0$; we set $M_{\lambda}=\bigcup_{j \in \mathbb{N}} \operatorname{Ker}_{M}(\Theta-\lambda)^{j}$.

Recall the following result proved in [39, Theorem 1.3] (which holds in the analytic case).

Theorem 5.13. Let $M$ be a homogeneous $\mathcal{D}(V)$-module. Then:

(1) $M=\mathcal{D}(V) . E$, E finite dimensional and generated by quasi-homogeneous elements;

(2) if $F M=\left(F_{p} M\right)_{p \in \mathbb{N}}$ is a $\Theta$-stable good filtration on $M$, the space $F_{p} M \cap M_{\lambda}$ is finite dimensional for all $p \in \mathbb{N}, \lambda \in \mathbb{C}$.

Using this result it is not difficult to obtain the next corollary.

Corollary 5.14. Let $M$ be a finitely generated $\mathcal{D}(V)$-module. The following assertions are equivalent:

(i) $M$ is monodromic;

(ii) $M$ is homogeneous;

(iii) $M=\mathcal{D}(V) . E, E$ finite dimensional such that $\Theta . E \subset E$.

Recall that a holonomic $\mathcal{D}(V)$-module is regular if there exists a good filtration $F M$ on $M$ such that the ideal $\operatorname{ann}_{\mathbb{C}\left[T^{*} V\right]} \operatorname{gr}^{F}(M)$ is radical, see [24, Corollary 5.1.11]. Denote by:

$-\bmod _{\tilde{\mathcal{C}}}^{\mathrm{rh}}\left(\mathcal{D}_{V}\right)$ the category of regular holonomic whose characteristic variety is contained in $\tilde{\mathcal{C}}(V)$;

$-\bmod _{\tilde{\mathcal{C}}}^{\Theta}\left(\mathcal{D}_{V}\right)$ the category of monodromic modules with characteristic variety contained in $\tilde{\mathcal{C}}(V)$.

Let $G_{1}$ be the simply connected cover of $G$ and set $\tilde{G}_{1}=G_{1} \times C$ (recall that $C \cong \mathbb{C}^{*}$ is the connected component of the centre of $\left.\tilde{G}\right)$. One has: $\operatorname{Lie}\left(\tilde{G}_{1}\right)=$ $\tilde{\mathfrak{g}}=\mathfrak{g} \oplus \mathfrak{c}, \mathfrak{c}=\mathbb{C} \zeta$, where $\tau(\zeta)=\bar{\Theta}$ as above. The group $\tilde{G}_{1}$ maps onto $G \times C$, and therefore onto $\tilde{G}=G C$. It follows that $\tilde{G}_{1}$ and $G \times C$ act on $V$; the orbits in $V$ then coincide with the $\tilde{G}$-orbits $O_{1}, \ldots, O_{t}$. The category of $\tilde{G}_{1}$-equivariant $\mathcal{D}(V)$-modules is denoted by

Observe that if

$$
\bmod ^{\tilde{G}_{1}}\left(\mathcal{D}_{V}\right)
$$

$$
\bmod ^{G \times C}\left(\mathcal{D}_{V}\right)
$$

is the category of $(G \times C)$-equivariant $\mathcal{D}(V)$-modules, any object in $\bmod ^{G \times C}\left(\mathcal{D}_{V}\right)$ can be naturally considered as an object of $\bmod ^{\tilde{G}_{1}}\left(\mathcal{D}_{V}\right)$. When $G$ is simply connected, e.g. $G=\mathrm{SL}(n), \mathrm{SL}(n) \times \mathrm{SL}(n), \mathrm{SL}(n) \times \mathrm{Sp}(m)$ or $\mathrm{G}_{2}$, we have $\tilde{G}_{1}=G \times C$ and these two categories are the same. 
Lemma 5.15. Let $M$ be a finitely generated $\mathcal{D}(V)$-module. Then:

(i) $M \in \bmod ^{\tilde{G}_{1}}\left(\mathcal{D}_{V}\right) \Longleftrightarrow$ (ii) $M \in \bmod _{\tilde{\mathcal{C}}}^{\mathrm{rh}}\left(\mathcal{D}_{V}\right) \Longrightarrow($ iii $) M \in \bmod _{\tilde{\mathcal{C}}}^{\Theta}\left(\mathcal{D}_{V}\right)$.

In particular, $\bmod ^{G \times C}\left(\mathcal{D}_{V}\right)=\bmod _{\tilde{\mathcal{C}}}^{\mathrm{rh}}\left(\mathcal{D}_{V}\right)$ when $G$ is simply connected.

Proof. (i) $\Rightarrow$ (ii): By [4, Theorem 12.11], or [17, §5], $M$ is regular holonomic. Its characteristic variety $\mathrm{Ch} M$ is therefore a $\tilde{G}$-stable subvariety of $T^{*} V$. Let $X$ be an irreducible component of Ch $M$; then $X$ is a Lagrangian conical closed $\tilde{G}$-stable subvariety of $T^{*} V$ and, if $\pi: T^{*} V \rightarrow V$ is the natural projection, [22, §5, Lemme 1], implies that $X=\overline{T_{\pi(X) \text { reg }}^{*} V}$. But it is easy to see that $\pi(X)^{\text {reg }}$ (the smooth locus of $\pi(X))$ is equal to $O_{j}$ for some $1 \leq j \leq t$. Hence $X=\mathcal{C}_{j}$ and $\mathrm{Ch} M \subset \tilde{\mathcal{C}}(V)$.

(ii) $\Rightarrow$ (iii) and (i): (We mimic the proof of [39, Proposition 1.6].) Choose a good filtration such that $I(M)=\operatorname{ann}_{\mathbb{C}\left[T^{*} V\right]} \operatorname{gr}^{F}(M)$ is radical. Since $\operatorname{Ch} \subset \subset \tilde{\mathcal{C}}(V)$, the principal symbols $\alpha=\sigma(\bar{\Theta})$ and $\sigma(\tau(\xi)), \xi \in \mathfrak{g}$, belong to $I(M)$, that is to say $\sigma(\tau(\xi)) \operatorname{gr}_{j}^{F}(M)=\alpha \operatorname{gr}_{j}^{F}(M)=(0) \subset \operatorname{gr}_{j+1}^{F}(M)$; in other words: $\bar{\Theta} \cdot F_{j} M=$ $\Theta . F_{j} M \subset F_{j} M$ and $\tau(\xi) . F_{j} M \subset F_{j} M$. In particular, $M$ is homogeneous, i.e. monodromic. Let $x \in M$. Since $\operatorname{dim} \mathbb{C}[\Theta] . x<\infty$ there exist $j \in M$ and $\lambda_{1}, \ldots, \lambda_{l}$ such that $x \in \sum_{i=1}^{l} F_{j} M \cap M_{\lambda_{i}}$. From $[\tau(\mathfrak{g}), \Theta]=0$ and $\tau(\mathfrak{g}) \cdot F_{j} M \subset F_{j} M$ it follows that $\tau(\mathfrak{g}) . F_{j} M \cap M_{\lambda_{i}} \subset F_{j} M \cap M_{\lambda_{i}}$; hence $U(\mathfrak{g}) . x$ is contained in the finite dimensional space $\sum_{i=1}^{l} F_{j} M \cap M_{\lambda_{i}}$, cf. Theorem 5.13. This shows that the action of $\tilde{\mathfrak{g}}$ on $M$ given by the $\tau(\xi), \xi \in \tilde{\mathfrak{g}}$, is locally finite. The formula

$$
\mathrm{e}^{t \xi} \cdot x=\exp (t \tau(\xi)) \cdot x=\sum_{k \geq 0} \frac{t^{k}}{k !} \tau(\xi)^{k} \cdot x
$$

then yields a rational action of $\tilde{G}_{1}$ on $M$ whose differential is given by multiplication by the elements $\tau(\xi)$. It remains to check that this action is compatible with the action of $\tilde{G}$ on $\mathcal{D}(V)$, which is an easy exercise.

Following $[39,40,41,42,43]$ we want to describe the category $\bmod _{\tilde{\mathcal{C}}}^{\mathrm{rh}}\left(\mathcal{D}_{V}\right)$ in terms of a category of modules over $U=R=\mathbb{C}[z, \delta, \theta]$. A finitely generated $U$-module $N$ is called monodromic if, for all $v \in N, \operatorname{dim}_{\mathbb{C}} \mathbb{C}[\theta] . v<\infty$. Denote by

$$
\bmod ^{\theta}(U)
$$

the category of monodromic modules. Observe that $N \in \bmod ^{\theta}(U)$ decomposes as

$$
N=\bigoplus_{\lambda \in \mathbb{C}} N_{\lambda}, \quad N_{\lambda}=\bigcup_{j \geq 0} \operatorname{Ker}_{N}(\theta-\lambda)^{j} .
$$

Recall that $[\theta, z]=z,[\theta, \delta]=-\delta, z \delta=b^{*}(\theta), \delta z=b(\theta)$ and that the roots of $b(-s)$ are $\lambda_{0}+1=1, \lambda_{1}+1, \ldots, \lambda_{n-1}+1$, cf. Theorem 3.1. We then obtain:

- $z \cdot N_{\lambda} \subset N_{\lambda+1}, \delta . N_{\lambda} \subset N_{\lambda-1}$;

- $\operatorname{dim} N_{\lambda}<\infty$ ( $N$ is finitely generated);

- $z \delta$, resp. $\delta z$, is bijective on $N_{\lambda}$ if and only if $\lambda \neq-\lambda_{j}$, resp. $\lambda \neq-\left(\lambda_{j}+1\right)$, therefore $z: N_{\lambda} \stackrel{\sim}{\longrightarrow} N_{\lambda+1}, \delta: N_{\lambda+1} \stackrel{\sim}{\longrightarrow} N_{\lambda}$ if $\lambda \neq-\left(\lambda_{j}+1\right)$.

From these properties it is easy to give a description of the category $\bmod ^{\theta}(U)$ in terms of "finite diagrams of linear maps" as in [39, 40, 41, 43].

Let $M \in \bmod ^{G \times C}\left(\mathcal{D}_{V}\right)$. Since the differential of the $G$-action on $M$ is given by $x \mapsto \xi . x, \xi \in \mathfrak{g}, x \in M$, one has:

$$
\Phi M=M^{G}=\{x \in M: \tau(\mathfrak{g}) \cdot x=0\} .
$$

Therefore $[\mathcal{D}(V) \tau(\mathfrak{g})]^{G} \cdot M=0$ and, via $\operatorname{rad}: \mathcal{D}(V)^{G} /[\mathcal{D}(V) \tau(\mathfrak{g})]^{G} \stackrel{\sim}{\longrightarrow}$ (Theorem 3.9), $\Phi M$ can be considered as a $U$-module by: $u . x=D . x$ if $u=\operatorname{rad}(D)$, $x \in M^{G}$. Observe also that the isomorphism rad yields a natural structure of 
right $U$-module on the module $\mathcal{N}=\mathcal{D}(V) / \mathcal{D}(V) \tau(\mathfrak{g})$ by: $\bar{a} \cdot u=\overline{a D}$ if $\bar{a} \in \mathcal{N}$ and $u=\operatorname{rad}(D) \in U$. For $N \in \bmod ^{\theta}(U)$ we set:

$$
\Psi N=\mathcal{N} \otimes_{U} N
$$

With these notation we have:

Proposition 5.16. (1) Let $M \in \bmod ^{G \times C}\left(\mathcal{D}_{V}\right)$ and $N \in \bmod ^{\theta}(U)$, then:

$$
\Phi M \in \bmod ^{\theta}(U), \quad \Psi N \in \bmod ^{G \times C}\left(\mathcal{D}_{V}\right), \quad \Phi \Psi N=N .
$$

(2) Suppose that any $M \in \bmod ^{G \times C}\left(\mathcal{D}_{V}\right)$ is generated by $M^{G}$ as a $\mathcal{D}(V)$-module. Then the categories $\bmod ^{G \times C}\left(\mathcal{D}_{V}\right)$ and $\bmod ^{\theta}(U)$ are equivalent via the functors $\Phi$ and $\Psi$. If furthermore $G$ is simply connected, we obtain: $\bmod _{\tilde{\mathcal{C}}}^{\mathrm{rh}}\left(\mathcal{D}_{V}\right) \equiv \bmod ^{\theta}(U)$.

Proof. (1) From $G$ reductive and $M$ finitely generated, one deduces that the $\mathcal{D}(V)^{G_{-}}$ module $M^{G}$ is finitely generated. Recall that $M$ is monodromic (Lemma 5.15); since $\theta . x=\operatorname{rad}(\bar{\Theta}) . x=\Theta . x$ it follows that $\Phi M$ is monodromic. Thus $\Phi N \in \bmod ^{\theta}(U)$.

It is clear that $\Psi N$ is finitely generated over $\mathcal{D}(V)$. The group $G$ acts naturally on $\mathcal{D}(V)$ and this action passes to $\mathcal{N}$ (note that $\mathcal{D}(V) \tau(\mathfrak{g})$ is $G$-stable). One easily checks that one can endow $\mathcal{N} \otimes_{U} N$ with a rational $G$-module structure by setting: $g .\left(\bar{a} \otimes_{U} x\right)=\overline{g . a} \otimes_{U} x$ for $\bar{a} \in \mathcal{N}, g \in G, x \in N$. Notice that since $N$ is monodromic the group $C=\exp (\mathbb{C} \zeta)$ acts on $N$ by $\mathrm{e}^{t \zeta} \cdot x=\exp (t \theta) \cdot x$. One can then verify that $C$ acts on $\mathcal{N} \otimes_{U} N$ by: $\mathrm{e}^{t \zeta} \cdot\left(\bar{a} \otimes_{U} x\right)=\mathrm{e}^{t \zeta} \cdot \bar{a} \otimes_{U} \exp (t \theta) \cdot x, t \in \mathbb{C}$. One shows without difficulty that this $G \times C$-action is compatible with the $\tilde{G}$-action on $\mathcal{D}(V)$. Moreover, with the previous notation we get that:

$$
\begin{aligned}
\frac{d}{d t}_{\mid t=0} \mathrm{e}^{t \xi} \cdot\left(\bar{a} \otimes_{U} x\right) & =\overline{[\tau(\xi), a]} \otimes_{U} x=\overline{\tau(\xi) a} \otimes_{U} x=\tau(\xi) \cdot\left(\bar{a} \otimes_{U} x\right), \\
\frac{d}{d t}_{\mid t=0} \mathrm{e}^{t \zeta} \cdot\left(\bar{a} \otimes_{U} x\right) & =\overline{[\bar{\Theta}, a]} \otimes_{U} x+\bar{a} \otimes_{U} \theta \cdot x=\overline{\bar{\Theta} a} \otimes_{U} x-\overline{a \bar{\Theta}} \otimes_{u} x+\bar{a} \otimes_{U} \theta . x \\
& =\bar{\Theta} \cdot\left(\bar{a} \otimes_{U} x\right)-\bar{a} \otimes_{U} \theta \cdot x+\bar{a} \otimes_{U} \theta \cdot x \\
& =\bar{\Theta} \cdot\left(\bar{a} \otimes_{U} x\right)=\tau(\zeta) \cdot\left(\bar{a} \otimes_{U} x\right) .
\end{aligned}
$$

This shows that $\Psi N \in \bmod ^{G \times C}\left(\mathcal{D}_{V}\right)$. The equality $(\Psi N)^{G}=\overline{1} \otimes_{U} N$ follows easily from the definition of the $G$-action on $\Psi N$, hence $\Phi \Psi N=N$.

(2) Note that there is a surjective $(G \times C)$-equivariant morphism of $\mathcal{D}(V)$-modules $\mathrm{m}: \Psi \Phi M=\mathcal{N} \otimes_{U} M^{G} \rightarrow M$ given by $\mathrm{m}\left(\bar{a} \otimes_{U} x\right)=a . x$. Set $L=$ Ker $\mathrm{m}$, hence $\mathrm{m}:(\Psi \Phi M) / L \stackrel{\sim}{\longrightarrow} M$. Then $L \in \bmod ^{G \times C}\left(\mathcal{D}_{V}\right)$ is generated by $L^{G}$, by hypothesis, and we obtain ( $G$ is reductive):

$$
M^{G} \cong(\Psi \Phi M / L)^{G}=(\Psi \Phi M)^{G} / L^{G}=(\Phi \Psi \Phi M) / L^{G}=M^{G} / L^{G} .
$$

This implies $L^{G}=(0)$, thus $L=0$ and $\Psi \Phi M \equiv M$. The last statement follows from Lemma 5.15

The previous proposition and work of P. Nang lead to the following:

Conjecture 5.17. Let $(\tilde{G}: V)$ be of Capelli type with $\operatorname{dim} V / / G=1$. Then the categories $\bmod ^{G \times C}\left(\mathcal{D}_{V}\right)$ and $\bmod ^{\theta}(U)$ are equivalent via the functors $\Phi$ and $\Psi$.

By Proposition 5.16, this conjecture is equivalent to showing that $M=\mathcal{D}(V) M^{G}$ for all $M \in \bmod ^{G \times C}\left(\mathcal{D}_{V}\right)$.

P. Nang [39, 41, 43] has proved Conjecture 5.17 in the cases: $\left(\mathrm{SO}(n) \times \mathbb{C}^{*}\right.$ : $\left.\mathbb{C}^{n}\right),\left(\mathrm{GL}(n) \times \mathrm{SL}(n): \mathrm{M}_{n}(\mathbb{C})\right),\left(\mathrm{GL}(2 m): \bigwedge^{2} \mathbb{C}^{2 m}\right)$. It would be interesting to obtain a uniform proof in the eight cases where $(\tilde{G}: V)$ is of Capelli type and $\operatorname{dim} V / / G=1$ (see Appendix A). As observed above the category $\bmod ^{\theta}(U)$ has a nice combinatorial description, which would give, when $G$ is simply connected, a 
classification of the regular holonomic modules on $V$ whose characteristic variety is contained in $\tilde{\mathcal{C}}(V)$. 


\begin{tabular}{|c|c|c|c|c|c|}
\hline & $(\tilde{G}: V)$ & $\operatorname{deg} f$ & $b(s)$ & Capelli & com. parabolic \\
\hline 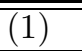 & 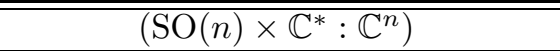 & $\overline{22}$ & $\overline{(s+1)(s+n / 2)}$ & yes & yes \\
\hline$(2)$ & $\left(\mathrm{GL}(n): S^{2} \mathbb{C}^{n}\right)$ & $n$ & $\mathrm{~T}_{i=1}^{n}(s+(i+1) / 2)$ & yes & yes \\
\hline$(3)$ & $\left(\mathrm{GL}(n): \bigwedge^{2} \mathbb{C}^{n}\right), n$ even & $n / 2$ & $\prod_{i=1}^{n / 2}(s+2 i-1)$ & yes & yes \\
\hline (4) & $\left(\mathrm{GL}(n) \times \mathrm{SL}(n): \mathrm{M}_{n}(\mathbb{C})\right)$ & $n$ & $\prod_{i=1}^{n}(s+i)$ & yes & yes \\
\hline$(5)$ & $\left(\mathrm{Sp}(n) \times \mathrm{GL}(2): \mathrm{M}_{2 n, 2}(\mathbb{C})\right)$ & 2 & $(s+1)(s+2 n)$ & yes & no \\
\hline$(6)$ & $\left(\mathrm{SO}(7) \times \mathbb{C}^{*}: \operatorname{spin}=\mathbb{C}^{8}\right)$ & 2 & $(s+1)(s+4)$ & yes & no \\
\hline (7) & $\left(\mathrm{SO}(9) \times \mathbb{C}^{*}: \operatorname{spin}=\mathbb{C}^{16}\right)$ & 2 & $(s+1)(s+8)$ & no & no \\
\hline$(8)$ & $\left(\mathrm{G}_{2} \times \mathbb{C}^{*}: \mathbb{C}^{7}\right)$ & 2 & $(s+1)(s+7 / 2)$ & yes & no \\
\hline$(9)$ & $\left(\mathrm{E}_{6} \times \mathbb{C}^{*}: \mathbb{C}^{27}\right)$ & 3 & $(s+1)(s+5)(s+9)$ & no & yes \\
\hline$(10)$ & $\left(\mathrm{GL}(4) \times \mathrm{Sp}(2): \mathrm{M}_{4}(\mathbb{C})\right)$ & 4 & $(s+1)(s+2)(s+3)(s+4)$ & yes & yes \\
\hline$\left(3^{\prime}\right)$ & $\left(\mathrm{GL}(n): \bigwedge^{2} \mathbb{C}^{n}\right), n$ odd & - & - & yes & no \\
\hline$\left(4^{\prime}\right)$ & $\left(\mathrm{GL}(n) \times \mathrm{SL}(m): \mathrm{M}_{n, m}(\mathbb{C})\right), n \neq m$ & - & - & yes & no \\
\hline$(11)$ & $\left(\mathrm{Sp}(n) \times \mathrm{GL}(1): \mathbb{C}^{2 n}\right)$ & - & - & yes & no \\
\hline$(12)$ & $\left(\mathrm{Sp}(n) \times \mathrm{GL}(3): \mathrm{M}_{2 n, 3}(\mathbb{C})\right)$ & - & - & no & no \\
\hline$\left(10^{\prime}\right)$ & $\left(\mathrm{GL}(n) \times \mathrm{Sp}(2): \mathrm{M}_{n, 4}(\mathbb{C})\right), n \neq 4$ & - & - & yes & no \\
\hline$(13)$ & $\left(\mathrm{SO}(10) \times \mathbb{C}^{*}: \frac{1}{2} \operatorname{spin}=\mathbb{C}^{16}\right)$ & - & - & yes & no \\
\hline
\end{tabular}




\section{REFERENCES}

[1] C. Benson and G. Ratcliff, On multiplicity free actions. In: Representations of real and p-adic groups, 221-304, Lect. Notes Ser. Inst. Math. Sci. Natl. Univ. Singap., 2, Singapore Univ. Press, World Scientific, Singapore, 2004.

[2] J.-E. Björk, Rings of Differential Operators, North Holland, Amsterdam, 1979.

[3] M.-P. Malliavin, Paris, 1987/1988, 137-173, Lecture Notes in Math., 1404, Springer, Berlin, 1989.

[4] A. Borel et al., Algebraic D-modules, Academic Press, Boston, 1987.

[5] J. Dadok and V. Kac, Polar representations, J. Algebra, 92 (1985), 504-524.

[6] M. de Jeu, Paley-Wiener theorems for the Dunkl transform, Trans. Amer. Math. Soc., 358 (2006), 4225-4250.

[7] C. F. Dunkl, Differential-difference operators associated to reflection groups, Trans. Amer. Math. Soc., 311 (1989), 167-183.

[8] C. F. Dunkl and E. Opdam, Dunkl operators for complex reflection groups, Proc. London Math. Soc., 86 (2003), 70-108.

[9] P. Etingof and V. Ginzburg, Symplectic reflection algebras, Calogero-Moser space, and deformed Harish-Chandra homomorphism, Invent. Math., 147 (2002), 243-348.

[10] R. Goodman and N. R. Wallach, Representations and invariants of the classical groups, Encyclopedia of Mathematics and its Applications, 68. Cambridge University Press, Cambridge, 1998.

[11] I. G. Gordon, Symplectic Reflection Algebras, Preprint (2007), ArXiv: math.RT/0712.1568.

[12] A. Grothendieck, Éléments de Géométrie Algébrique - Chapitre IV, Inst. Hautes Etudes Sci. Publ. Math., 32 (1967).

[13] A. Gyoja, Theory of Prehomogeneous Vector Spaces without Regularity Condition, Publ. Res. Inst. Math. Sci., 27 (1991), 861-922.

[14] G. J. Heckman, A remark on the Dunkl differential-difference operators. In: "Harmonic analysis on reductive groups", Progress in mathematics 101, Birkhäuser, 1991.

[15] S. Helgason, Differential Geometry, Lie Groups and Symmetric Spaces, Academic Press, 1978.

[16] R. Hotta, Introduction to D-modules. Lectures at the Inst. Math. Sci., Madras, Math. Institute, Tohoku University, Sendai, 1986.

[17] Equivariant D-modules, Preprint (1998), ArXiv: math.RT/9805021.

[18] R. Howe and T. Umeda, The Capelli identity, the double commutant theorem and multiplicity free actions, Math. Annalen, 290 (1991), 565-619.

[19] X. Jie and F. Van Oystaeyen, Weight Modules and their Extensions over a Class of Algebras Similar to the Enveloping Algebra of sl(2, C), J. Algebra, 175 (1995), 844-864.

[20] A. Joseph, A generalization of Quillen's lemma and its application to the Weyl algebras, Israel J. Math., 28 (1977), no. 3, 177-192.

[21] V. G. Kac, Some Remarks on Nilpotent Orbits, J. Algebra, 64 (1980), 190-213.

[22] M. Kashiwara, $B$-functions and holonomic systems. Rationality of roots of $B$-functions, Invent. Math., 38 (1976/77), no 1, 33-53.

[23] - Systèmes d'équations micro-différentielles. Notes de T. Monteiro Fernandes, 1976/77. Pré-publications de l'Université Paris-Nord, Paris, 1979. English version: Systems of Micro-differential Operators, Progress in Math. 34, Birkhaüser, 1983.

[24] M. Kashiwara and T. Kawai, On holonomic systems of micro-differential equations III Systems with regular singularities, Publ. Res. Inst. Math. Sci., 17 (1981), 813-979.

[25] T. Kimura, Introduction to Prehomogeneous Vector Spaces, Translations of Mathematical Monographs, vol. 215, AMS, Providence, 2003.

[26] F. Knop, Some remarks on multiplicity free spaces. In: Proc. NATO Adv. Study Inst. on Representation Theory and Algebraic Geometry (A. Broer, A. Daigneault, G. Sabidussi, eds.), 301-317, Nato ASI Series C, Vol. 514, Dortrecht, Kluwer, 1998.

[27] A. Leahy, A classification of multiplicity free representations, J. Lie Theory, 8 (1998), 367391.

[28] T. Levasseur and J. T. Stafford, Invariant Differential Operators and an Homomorphism of Harish-Chandra, J. Amer. Math. Soc., 8 (1995), 365-372.

[29] - Semi-simplicity of invariant holonomic systems on a reductive Lie algebra, Amer. J. Math., 119 (1997), 1095-1117.

[30] - Invariant differential operators on the tangent space of some symmetric spaces, Ann. Inst. Fourier, 49 (1999), 1711-1741.

[31] _ Radial Components of Invariant Differential Operators on the Tangent Space of Symmetric Spaces, in preparation. 
[32] J. C. McConnell and J. C. Robson, Noncommutative Noetherian Rings, Grad. Texts in Math. Vol. 30, Amer. Math. Soc., Providence, RI, 2000.

[33] M. Muro, Singular Invariant Tempered Distributions on Regular Prehomogeneous Vector Spaces, J. Funct. Anal., 76 (1988), 317-345.

[34] _ Invariant Hyperfunctions on Regular Prehomogeneous Vector Spaces of Commutative Parabolic Type, Tôhoku Math. J., 42 (1990), 163-193.

[35] , Singular invariant hyperfunctions on the space of real symmetric matrices, Tohoku Math. J., 51 (1999), 329-364.

[36] Invariant hyperfunction solutions to invariant differential equations on the space of real symmetric matrices, J. Funct. Anal., 193 (2002), 346-384.

[37] Singular invariant hyperfunctions on the square matrix space and the alternating matrix space, Nagoya Math. J., 169 (2003), 19-75.

[38] I. M. Musson and M. Van den Bergh, Invariants under tori of rings of differential operators and related topics, Mem. Amer. Math. Soc., 136, 1998.

[39] P. Nang, D-modules associated to the group of similitudes, Publ. Res. Inst. Math. Sci., 35 (1999), 223-247.

[40] $\mathcal{D}$-modules associated to $3 \times 3$ matrices C. R. Math. Acad. Sci. Paris, 338 (2004), $139-144$.

[41] $\mathcal{D}$-modules associated to the determinantal singularities, Proc. Japan Acad. Ser. A Math. Sci., 80 (2004), 74-78.

[42] Algebraic description of $\mathcal{D}$-modules associated to $3 \times 3$ matrices, Bull. Sci. Math., 130 (2006), 15-32.

[43] Regular holonomic $\mathcal{D}$-modules on skew symmetric matrices, Preprint of the Max Planck Institut für Mathematik, MPIM2007-42 (2007).

[44] D. I. Panyushev, The Jacobian modules of a representation of a Lie algebra and geometry of commuting varieties, Compositio Math., 94 (1994), 181-199.

[45] 185-202.

[46] V. L. Popov and E. B. Vinberg, Invariant Theory. In: "Algebraic Geometry IV", (Eds: A. N. Parshin and I. R. Shafarevich), Springer-Verlag, Berlin/Heidelberg/New York, 1991.

[47] V. S. Pyasetskii, Linear Lie groups acting with finitely many orbits, Functional. Anal. App., 9 (1975), 351-353.

[48] M. Rausch de Traubenberg, M. Slupinski and A. Tanasă, Finite-dimensional Lie subalgebras of the Weyl algebra, J. Lie Theory, 16 (2006), 427-454.

[49] H. Rubenthaler, Une dualité de type Howe en dimension infinie, C. R. Acad. Sci. Paris Sér. I Math., 314 (1992), no. 6, 435-440.

[50] G. W. Schwarz, Lifting differential operators from orbit spaces, Ann. Sci. École Norm. Sup., 28 (1995), 253-306.

[51] Finite-dimensional representations of invariant differential operators, J. Algebra, 258 (2002), 160-204

[52] S. P. Smith, A class of algebras similar to the enveloping algebra of $\mathfrak{s l}(2)$, Trans. Amer. Math.Soc, 322 (1990), 285-314.

[53] C. Torossian, Une application des opérateurs de Dunkl au théorème de restriction de Chevalley, C. R. Acad. Sci. Paris, 318 (1994), 895-898.

[54] V. S. Varadarajan, Harmonic Analysis on Real Reductive Groups, Lecture Notes in Mathematics, 576, Springer-Verlag, Berlin/New York, 1977.

[55] M. Van den Bergh and F. Van Oystaeyen, Lifting maximal orders, Comm. in Algebra, 17 (1989), 341-349.

Laboratoire de Mathématiques, UMR6205, Université de Brest, 29238 Brest Cedex 3, FRANCE

E-mail address: Thierry.Levasseur@univ-brest.fr 